\title{
Isothiourea-Mediated Asymmetric Functionalization of 3-Alkenoic Acids
}

\author{
Louis C. Morrill, Samuel M. Smith, Alexandra M. Z. Slawin and Andrew D. Smith* \\ EaStCHEM, School of Chemistry, University of St Andrews, North Haugh, St Andrews, KY16 9ST, \\ U.K.
}

e-mail: ads10@st-andrews.ac.uk

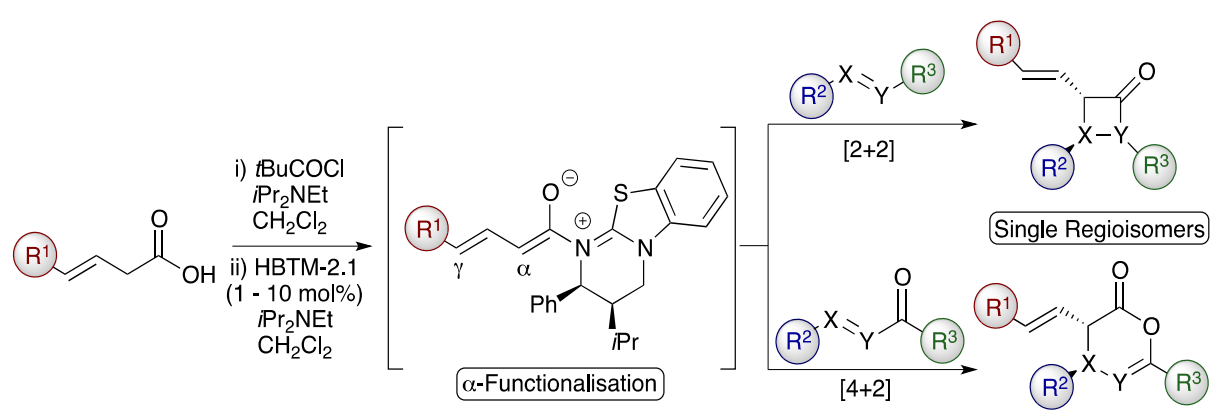

\begin{abstract}
:
Isothiourea HBTM-2.1 promotes the catalytic asymmetric $\alpha$-functionalization of 3-alkenoic acids through formal [2+2] cycloadditions with $N$-tosyl aldimines and formal [4+2] cycloadditions with either 4aryltrifluoromethyl enones or $\mathrm{N}$-aryl- $\mathrm{N}$-aroyl diazenes, providing useful synthetic building blocks in good yield and with excellent enantiocontrol (up to $>99 \%$ ee). Stereodefined products are amenable to further synthetic elaboration through manipulation of the olefinic functionality.
\end{abstract}




\section{Introduction}

The organocatalytic generation of dienolates or their dienamine equivalents is an increasingly popular area of research. ${ }^{1}$ These intermediates have powerful synthetic potential due to their ability to react regio- and enantioselectively through either $\alpha$ - or $\gamma$-positions, allowing rapid access to diverse molecular scaffolds. In particular, recent research has demonstrated the ability of ammonium and azolium dienolates to participate in asymmetric transformations. ${ }^{2-11}$ For example, Peters ${ }^{2}$ and $\mathrm{Ye}^{3}$ have accessed cinchona alkaloid and norephedrine derived $\mathrm{C} 1$-ammonium dienolates from $\alpha, \beta$-unsaturated acid chloride starting materials and applied these towards the synthesis of a range of stereodefined products (Scheme 1). C1-ammonium dienolate $\mathbf{1}$ may form either via initial dehydrohalogenation to form vinyl ketene $\mathbf{2}$ which is intercepted by the Lewis base, or from initial attack of the Lewis base to form $\alpha, \beta$-acyl ammonium 3 followed by $\gamma$ deprotonation. To the best of our knowledge, all catalytically generated $\beta, \beta$-disubstituted $\mathrm{C} 1$-ammonium dienolates documented in the literature react to give $\gamma$-functionalized products.

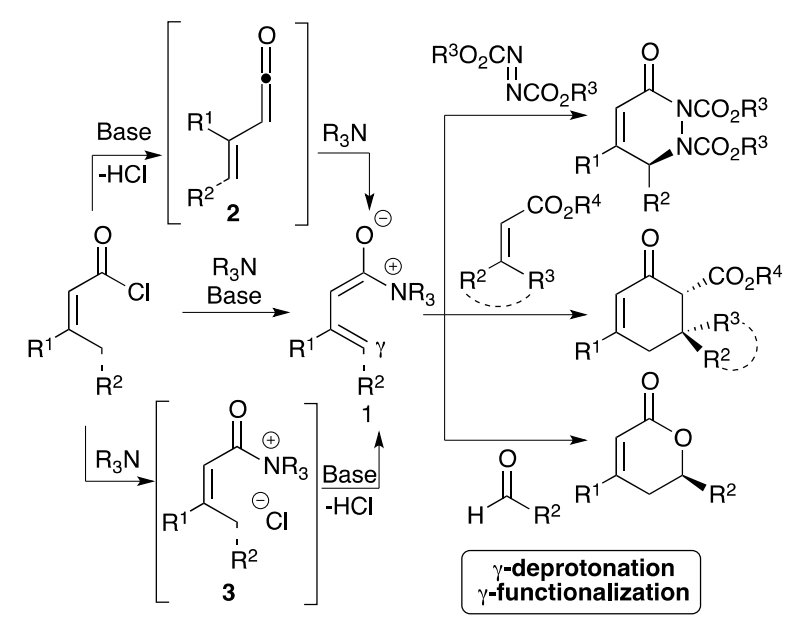

Scheme 1: Generation and utility of C1-ammonium dienolates.

C1-azolium dienolates have also received considerable attention within the past two years. For example, Ye demonstrated that $\alpha, \beta$-unsaturated acid chlorides $\mathbf{4}$, in the presence of an $\mathrm{N}$-heterocyclic carbene (NHC) and base, afford C1-azolium dienolates 5 that react via the $\gamma$-centre in asymmetric formal [4+2] 
cycloadditions with $2 \pi$ electrophiles (Scheme $2 a) .{ }^{4}$ Chi subsequently disclosed the ability to access the same dienolate via both enals 6 (in presence of a stoichiometric oxidant) ${ }^{5,6}$ and $\alpha, \beta$-unsaturated esters 7 (Scheme 2b,c). ${ }^{7}$ Alternatively, enals bearing an $\alpha$-bromo leaving group such as $\mathbf{8}$ have also been demonstrated as suitable azolium dienolate precursors (Scheme $2 \mathrm{~d}) .{ }^{8}$ In examples a-d, each process is postulated to involve $\gamma$-deprotonation of the corresponding $\beta, \beta$-disubstituted- $\alpha, \beta$-acyl azolium intermediate to generate the corresponding dienolate, often depicted in both $(E)$ - and $(Z)$-configurations, followed by $\gamma$-functionalization of the resulting azolium dienolate. ${ }^{9}$ Alternatively, aldehydes that contain a $\gamma$-leaving group such as $\mathbf{9}$ have been used to access C1-azolium dienolates $\mathbf{1 0}$ (Scheme 2e). Interestingly, these dienolates give $\alpha$-functionalization via fluorination with NFSI, and $\gamma$-functionalization in the formal [4+2] cycloaddition with diazodicarboxylates $\mathbf{1 1}$, affording esters $12^{10}$ and lactams $13^{11}$ respectively.

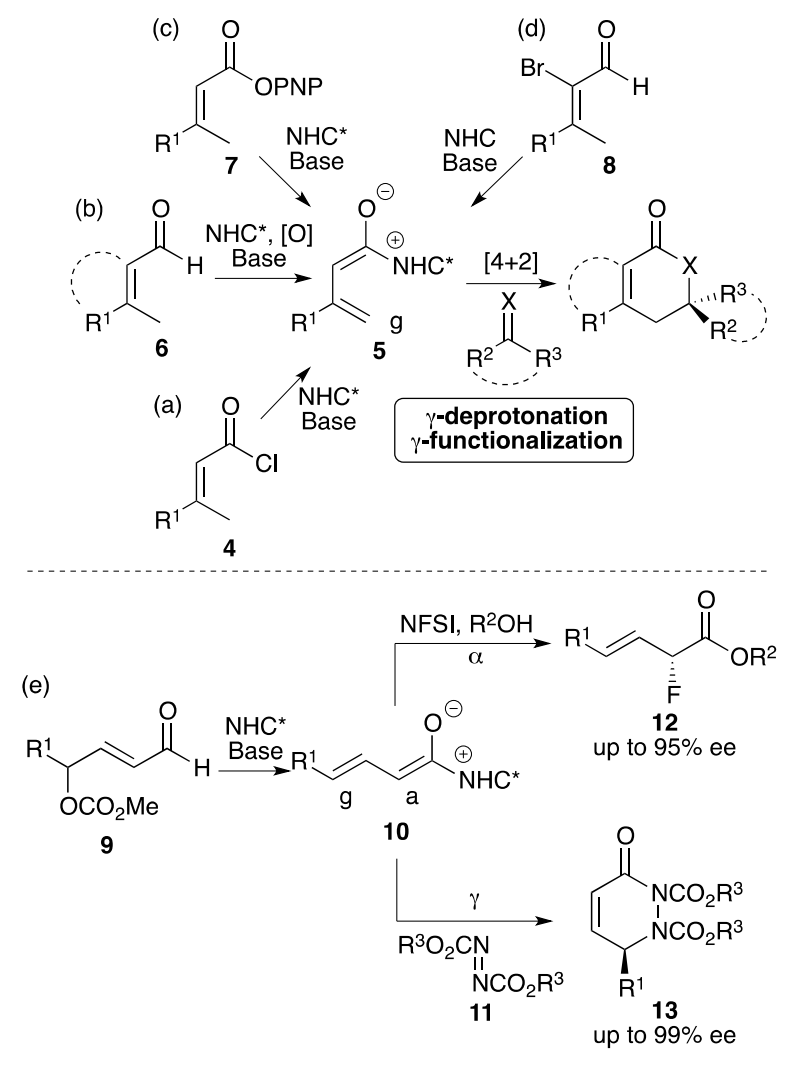

Scheme 2: Generation and utility of $\mathrm{C} 1$-azolium dienolates. 
Building upon the elegant nucleophile catalysed aldol-lactonisation (NCAL) reaction developed by Romo, ${ }^{12}$ we have recently shown that isothioureas ${ }^{13,14}$ can generate ammonium enolates ${ }^{15}$ in situ from carboxylic acids that subsequently undergo a range of intra- and intermolecular Michael additionlactonization/lactamization reactions to generate stereodefined products. ${ }^{16}$ Although powerful, the success of the intermolecular processes often relies upon using arylacetic acids as starting materials, ${ }^{17,18}$ which constitutes a limitation of this organocatalytic strategy. To broaden the substrate scope of such processes, the use of 3-alkenoic acids would allow access to extended ammonium dienolates that could give rise to either $\alpha$ - or $\gamma$-functionalized products in a stereodefined manner (Scheme 3 ). In this manuscript, a range of 3-alkenoic acids are shown to act as suitable precursors to isothiourea derived ammonium dienolates that react in a variety of formal [2+2] and [4+2] cycloadditions. In contrast to previously accessed C1ammonium dienolates formed via $\gamma$-deprotonation, these ammonium dienolates are formed via $\alpha$ deprotonation and provide exclusively $\alpha$-functionalized products. This strategy introduces an additional exocyclic olefin functional handle, allowing for further product functionalization into useful synthetic building blocks.

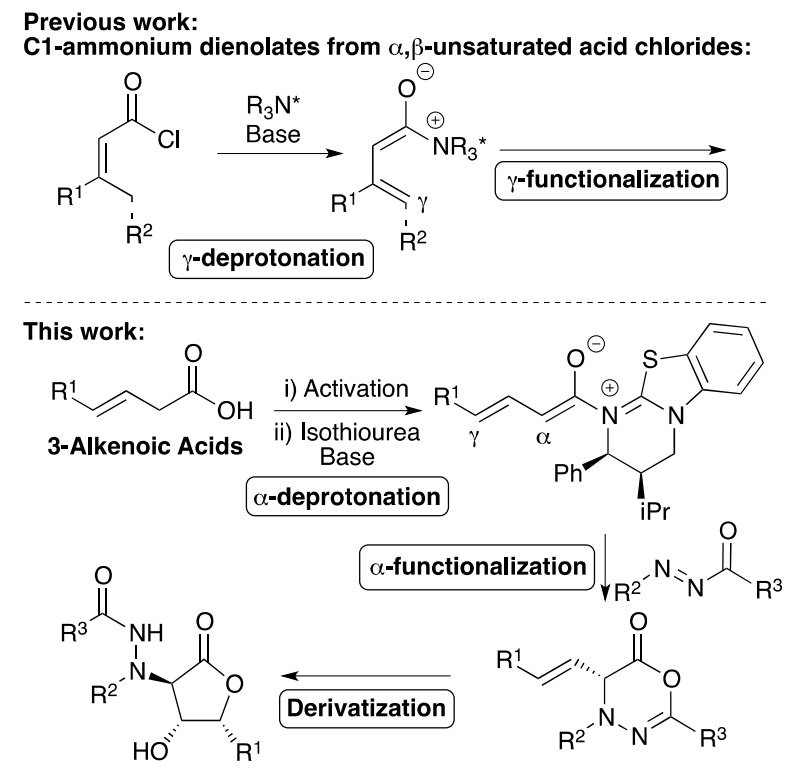

Scheme 3: Proposed asymmetric transformations of 3-alkenoic acids via either $\alpha$ - or $\gamma$-functionalization.

\section{Results and Discussion}




\section{Generation of isothiourea derived ammonium dienolates and reaction with $2 \pi$ electrophiles}

Initial studies probed the ability of isothioureas to generate an ammonium dienolate from a 3-alkenoic acid, with subsequent reaction with a reactive $2 \pi$ component used to test if $\alpha$ - or $\gamma$-selectivity is observed. Encouraged by Ye's report demonstrating diazadicarboxylates as suitable reaction partners with C1ammonium dienolates, ${ }^{3 a}$ along with both Ye and Chi who showed that trifluoromethyl ketones are suitable partners in $[4+2]$ cycloadditions with $\mathrm{C} 1$-azolium dienolates, ${ }^{4,5}$ these $2 \pi$ components were evaluated. Following our report in the previous manuscript we also evaluated $N$-tosyl aldimines $2 \pi$ electrophiles. ${ }^{19}$ Using 3-methylbut-3-enoic acid 14 or 2-(cyclopent-1-en-1-yl)acetic acid 15 with pivaloyl chloride as activating agent, achiral DHPB 17 (3,4-dihydro-2H-pyrimido[2,1-b]benzothiazole) as catalyst and trifluoromethyl ketone $\mathbf{1 8}$ as the $2 \pi$ electrophile, ${ }^{20}$ no distinguishable cycloaddition products were observed (Table 1). Under the same reaction conditions, $(E)-4$-phenylbut-3-enoic acid 16 reacted with trifluoromethyl ketone 18, giving solely the [2+2] cycloaddition product $\beta$-lactone $21(60: 40 \mathrm{dr}$ anti:syn) derived from $\alpha$-functionalization ${ }^{21}$ in $71 \%$ combined yield whilst reaction with $N$-tosyl aldimine 19 gave $\beta$-lactam 22 (83:17 dr anti:syn) in 68\% yield (anti diastereoisomer). ${ }^{22-24}$ Unfortunately, diazodicarboxylate $\mathbf{2 0}$ proved incompatible with this system giving no distinguishable product despite full consumption of 20.

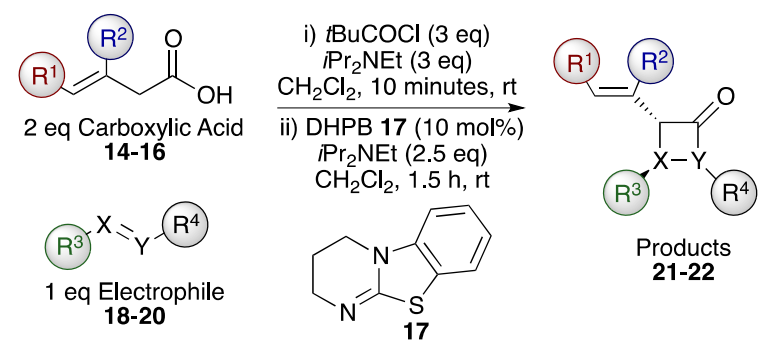

\begin{tabular}{|c|c|c|c|c|c|}
\hline Entry & Carboxylic Acid & Electrophile & Product (major) & $\mathrm{dr}^{\mathrm{a}}$ (anti:syn) & Yield $^{\mathrm{b}}$ (\% anti,syn) \\
\hline 1 & & & None & N/A & N/A \\
\hline 2 & & & None & N/A & N/A \\
\hline
\end{tabular}




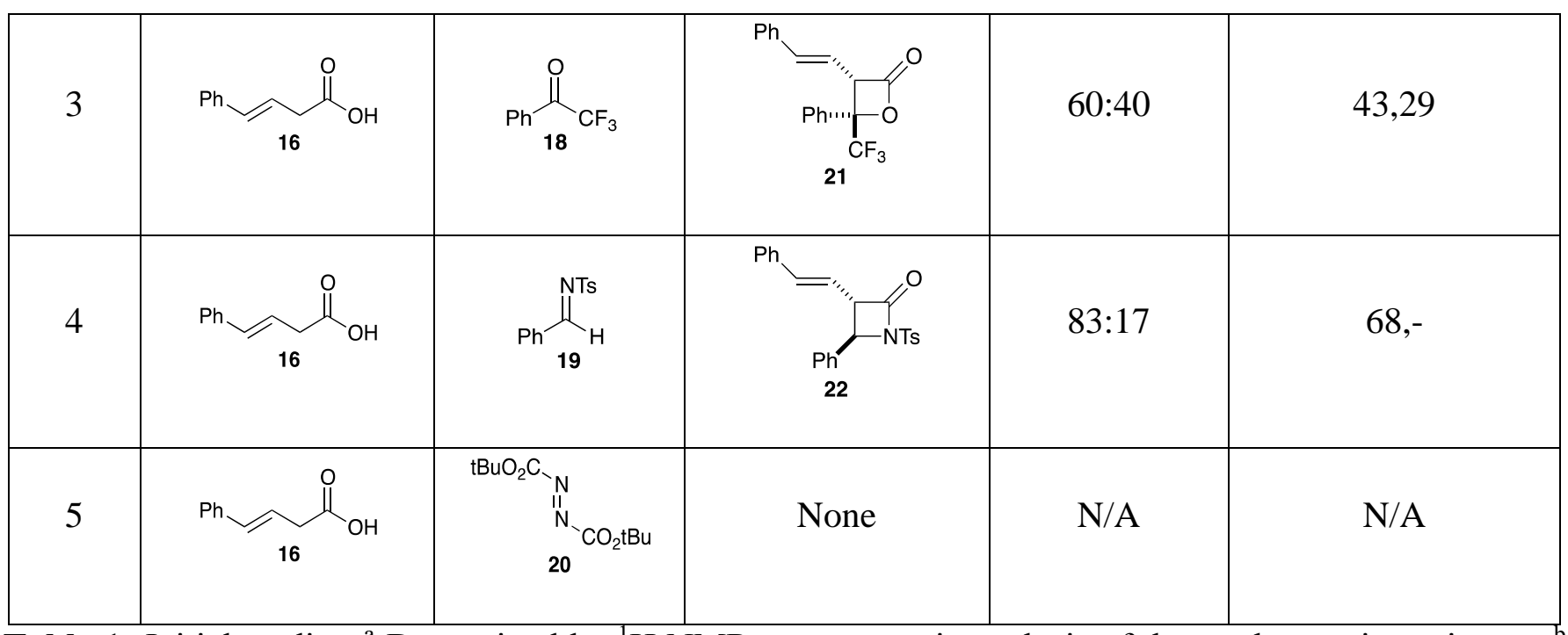

Table 1: Initial studies: ${ }^{a}$ Determined by ${ }^{1} \mathrm{H}$ NMR spectroscopic analysis of the crude reaction mixture;

Isolated yield ( $\geq 95: 5 \mathrm{dr})$.

Encouraged by the promising diastereoselectivities observed in the reaction with $N$-tosyl aldimine 19, further optimization studies focused upon finding a suitable asymmetric variant. Screening of a range of isothiourea catalysts and C(4)-substituted alkenoic acids revealed that chiral isothiourea HBTM-2.1 23 efficiently promotes the formal [2+2] cycloaddition of (E)-pent-3-enoic acid 24 and imine 25 at rt, affording $\beta$-lactam 26 in moderate diastereoselectivity (68:32 dr anti:syn), with each separable diastereoisomer isolated in good yield (53\% anti, 27\% syn) and enantioselectivity (anti 79\% ee, syn 72\% ee) (Table 2). Lowering the temperature to $-78{ }^{\circ} \mathrm{C}$ resulted in similar diastereoselectivity $(71: 29 \mathrm{dr}$ anti:syn), but with each separable diastereoisomer formed in excellent enantioselectivity (anti $97 \%$ ee, syn $>99 \% e e) .^{25}$

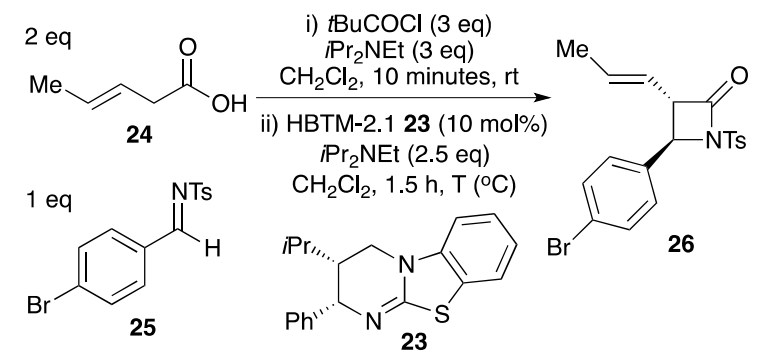

\begin{tabular}{|l|l|l|l|l|}
\hline Entry & $\mathrm{T}\left({ }^{\circ} \mathrm{C}\right)$ & $\mathrm{dr}^{\mathrm{a}}$ (anti:syn) & Yield $^{\mathrm{b}}$ (\% anti,syn) & $\mathrm{ee}^{\mathrm{c}}$ (\% anti,syn $)$ \\
\hline
\end{tabular}




\begin{tabular}{|c|c|c|c|c|}
\hline 1 & 23 & $68: 32$ & 53,27 & 79,72 \\
\hline 2 & -78 & $71: 29$ & 53,11 & $97,>99$ \\
\hline
\end{tabular}

Table 2: Formal [2+2] cycloaddition using $N$-tosyl aldimine 25: ${ }^{a}$ Determined by ${ }^{1} \mathrm{H}$ NMR spectroscopic analysis of the crude reaction mixture; ${ }^{\mathrm{b}}$ Isolated yield ( $\left.\geq 95: 5 \mathrm{dr}\right){ }^{\mathrm{c}}$ Determined by chiral HPLC analysis.

The absolute configuration of syn- $\beta$-lactam 27 was confirmed unambiguously by X-ray crystallography as $(3 S, 4 S),{ }^{26}$ while that of the anti- $\beta$-lactam 26 was confirmed by an epimerization experiment (Scheme 4). Treatment of syn- $\beta$-lactam $(3 S, 4 S)-27\left(>99: 1 \mathrm{dr},>99 \%\right.$ ee) using $i \mathrm{Pr}_{2} \mathrm{NEt}$ at $\mathrm{rt}$ for $16 \mathrm{~h}$ gave a $(58: 42$ syn:anti) mixture comprising of syn- $\beta$-lactam $(3 S, 4 S)-27(96 \%$ ee) and anti- $\beta$-lactam $(3 R, 4 S)-26(96 \%$ ee $)$ as determined by Chiral HPLC. The absolute configuration of the anti- $\beta$-lactam formed by epimerization is opposite to that observed experimentally in the catalytic process. Assuming epimerization occurs solely at $\mathrm{C}(3)$, this allows the absolute configuration of the anti- $\beta$-lactam formed in Table 2 to be assigned $(3 S, 4 R) .^{27}$

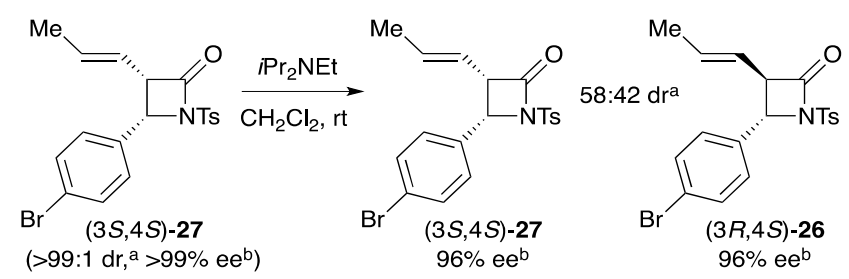

Scheme 4: Epimerization experiment; ${ }^{\text {a }}$ Determined by ${ }^{1} \mathrm{H}$ NMR spectroscopic analysis of the crude reaction mixture; ${ }^{b}$ Determined by HPLC analysis.

The generality of this protocol was next investigated by variation of both the acid and aldimine components (Table 3). Within the aldimine, electron-donating and withdrawing groups can be incorporated provided the reactions are carried out at $\mathrm{rt}^{28}$ 4-OMe Substituted $\beta$-lactam 28 is formed in good diastereo- and enantioselectivity, whilst incorporation of the $4-\mathrm{CF}_{3}$ group results in a significant reduction in enantioselectivity (44\% ee). Heteroaryl substituents (2-furyl) and extended aromatics (2naphthyl) are tolerated within the aldimine, giving $\beta$-lactams $\mathbf{3 0}$ and $\mathbf{3 1}$ in modest diastereoselectivity with 
the major (anti) diastereoisomer formed in excellent ee (95\% and $97 \%$ respectively). In cases where the minor (syn) diastereoisomer can be isolated, it is always formed in excellent enantioselectivity ( $>96 \%$ ee). Both the 4-position substituent and the configuration within the acid component can also be varied; for example (E)-4-ethyl, $(E)$-4-isopropyl and (E)-4-benzyl alkenoic acids give the corresponding $\beta$-lactams 32-34 in high yields and good diastereo- and enantioselectivities. Finally, (Z)-pent-3-enoic acid was used as a starting material, giving the usual $\alpha$-functionalization under the reaction conditions, but generating $\beta$ lactam 35 with negligible diastereoselectivity at $-78{ }^{\circ} \mathrm{C}(43: 57 \mathrm{dr}$, anti:syn), despite both diastereoisomers being formed in exquisite enantioselectivity $\left(>99 \%\right.$ ee).$^{29}$
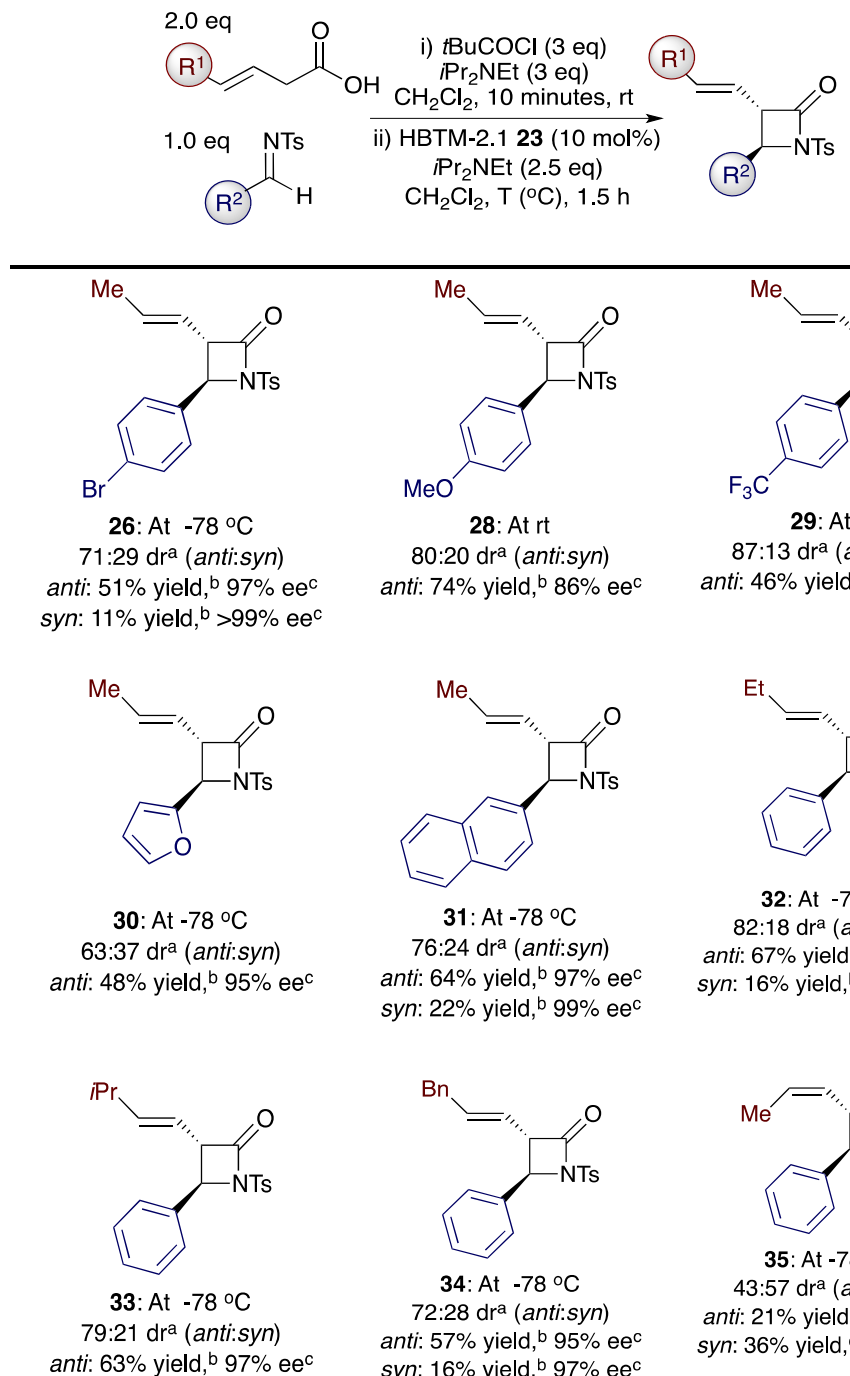

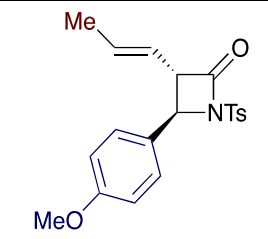

28: At rt

$80: 20 \mathrm{dr}^{\mathrm{a}}$ (anti:syn) anti: $74 \%$ yield, ${ }^{b} 86 \%$ ee

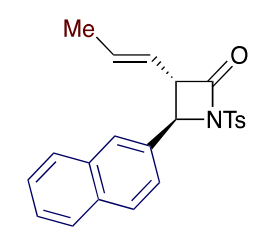

31: At $-78^{\circ} \mathrm{C}$ $76: 24 \mathrm{dr}^{\mathrm{a}}($ anti:syn) anti: $64 \%$ yield,,${ }^{b} 97 \%$ ee $^{c}$ syn: $22 \%$ yield, ${ }^{\mathrm{b}} 99 \%$ ee

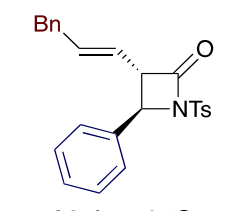

34: At $-78^{\circ} \mathrm{C}$ $72: 28 \mathrm{dr}^{\mathrm{a}}$ (anti:syn) anti: $57 \%$ yield, ${ }^{\mathrm{b}} 95 \% \mathrm{ee}^{\mathrm{c}}$ syn: $16 \%$ yield, ${ }^{b} 97 \% \mathrm{ee}^{\mathrm{c}}$

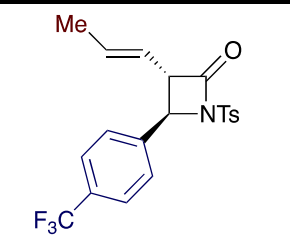

29: At rt

$87: 13 \mathrm{dr}^{\mathrm{a}}$ (anti:syn) anti: $46 \%$ yield, ${ }^{\text {b }} 44 \%$ ee $^{\mathrm{c}}$

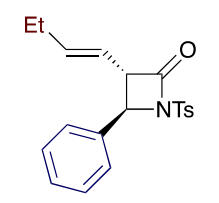

32: At $-78^{\circ} \mathrm{C}$ $82: 18 \mathrm{dr}^{\mathrm{a}}$ (anti:syn) anti: $67 \%$ yield, ${ }^{b} 98 \%$ ee $^{c}$ syn: $16 \%$ yield, ${ }^{b}>99 \%$ ee $^{c}$

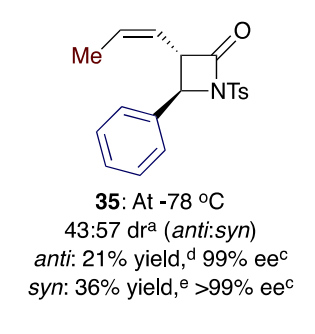


Table 3: Formal [2+2] cycloaddition scope. ${ }^{\text {a }}$ Determined by ${ }^{1} \mathrm{H}$ NMR spectroscopic analysis of the crude reaction mixture; ${ }^{\mathrm{b}}$ Isolated yield $(\geq 95: 5 \mathrm{dr}) ;{ }^{\mathrm{c}}$ Determined by chiral HPLC analysis; ${ }^{\mathrm{d}}$ Isolated yield $(92: 8$ $\mathrm{dr}){ }^{\mathrm{e}}$ Isolated yield $(88: 12 \mathrm{dr})$;

\section{$[4+2]$ cycloadditions of isothiourea derived ammonium dienolates with $4 \pi$ electrophiles}

Having established the propensity of these ammonium dienolates to react at the $\alpha$-position with $2 \pi$ electrophiles, their ability to partake in formal [4+2] cycloadditions with electron deficient $4 \pi$ Michael acceptors was investigated. HBTM-2.1 23 efficiently catalyzes the reaction between $(E)$-pent-3-enoic and (E)-1,1,1-trifluoro-4-phenyl-3-buten-2-one in only 5 minutes at $\mathrm{rt}$, giving $\delta$-lactone 36 in $80 \%$ yield with good diastereoselectivity $(88: 12 \mathrm{dr})$ and excellent enantioselectivity $\left(96 \%\right.$ ee).$^{30-32}$ The reaction proceeds efficiently using (E)-3-hexenoic acid, giving $\delta$-lactone 37, although when using $(E)$-styrylacetic acid the reaction has to be carried out at $-78{ }^{\circ} \mathrm{C}$ to prevent product decomposition and gives the major diastereoisomer of $\delta$-lactone 38 in reduced enantioselectivity (60\% ee). Heteroaryl and 4-bromophenyl substituted trifluoromethyl enones are also tolerated giving $\delta$-lactones $\mathbf{3 9}$ and $\mathbf{4 0}$ in good yields and high diastereo- and enantioselectivity (Table 4).
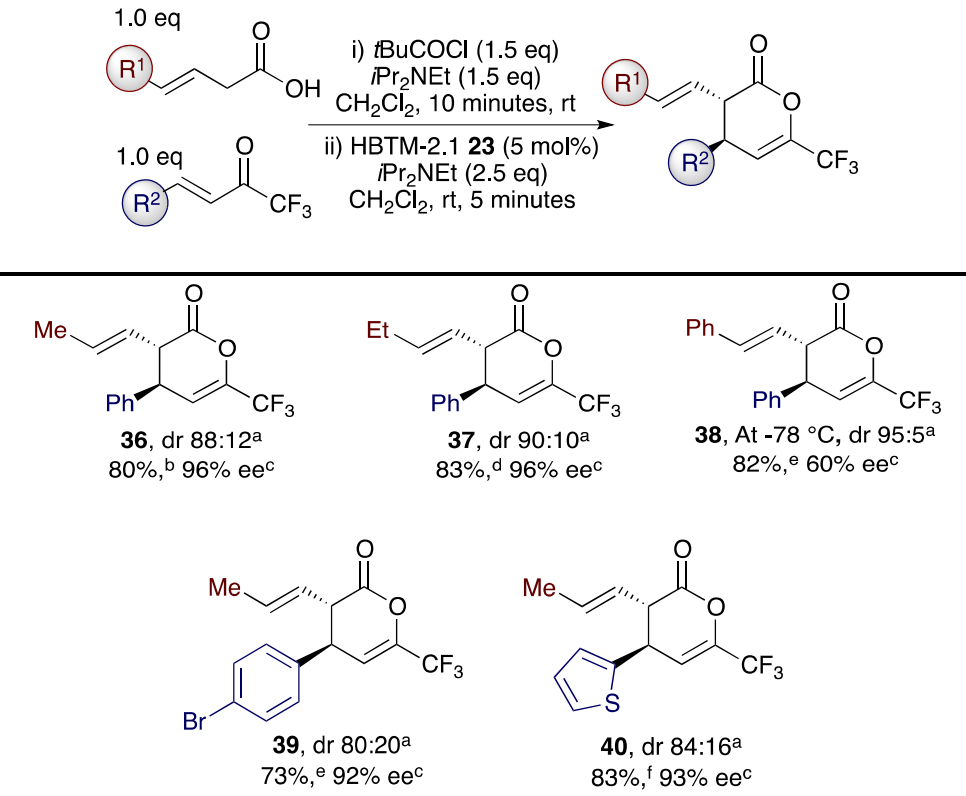
Table 4: Formal [4+2] cycloadditions with 4-aryltrifluoromethyl enones; ${ }^{\text {a }}$ Determined by ${ }^{1} \mathrm{H}$ NMR spectroscopic analysis of the crude reaction mixture; ${ }^{\mathrm{b}}$ Isolated yield $(88: 12 \mathrm{dr}){ }^{\mathrm{c}}$ Determined by HPLC analysis; ${ }^{\mathrm{d}}$ Isolated yield (93:7 dr); ${ }^{\mathrm{e}}$ Isolated yield ( $\left.\geq 95: 5 \mathrm{dr}\right) ;{ }^{\mathrm{f}}$ Isolated yield $(84: 16 \mathrm{dr})$.

The generality of this asymmetric Michael addition-lactonization process was next investigated using $N$ aryl- $N$-aroyldiazenes as Michael acceptors, followed by in situ ring opening of the intermediate 1,3,4oxadiazin-6-one formal [4+2] cycloaddition adduct with $\mathrm{MeOH}$. Examples including the use of 3-alkenoic acids bearing 4-alkyl (Me, Et, $i \operatorname{Pr})$, 4-benzyl and 4-phenyl substituents, in addition to $(E)$ - and $(Z)$-alkene configurations are all readily incorporated giving, after in situ ring-opening with methanol, ${ }^{33}$ a range of hydrazides 41-46 in high yields (71-85\%) and excellent enantioselectivity (91-99\% ee) (Table 5). ${ }^{34,35}$ Diazenes bearing electron deficient $\left(4-\mathrm{FC}_{6} \mathrm{H}_{4}\right)$ and heteroaryl (2-furyl) $N$-aroyl substituents are also tolerated giving products $\mathbf{4 7}$ and $\mathbf{4 8}$ in excellent ee.
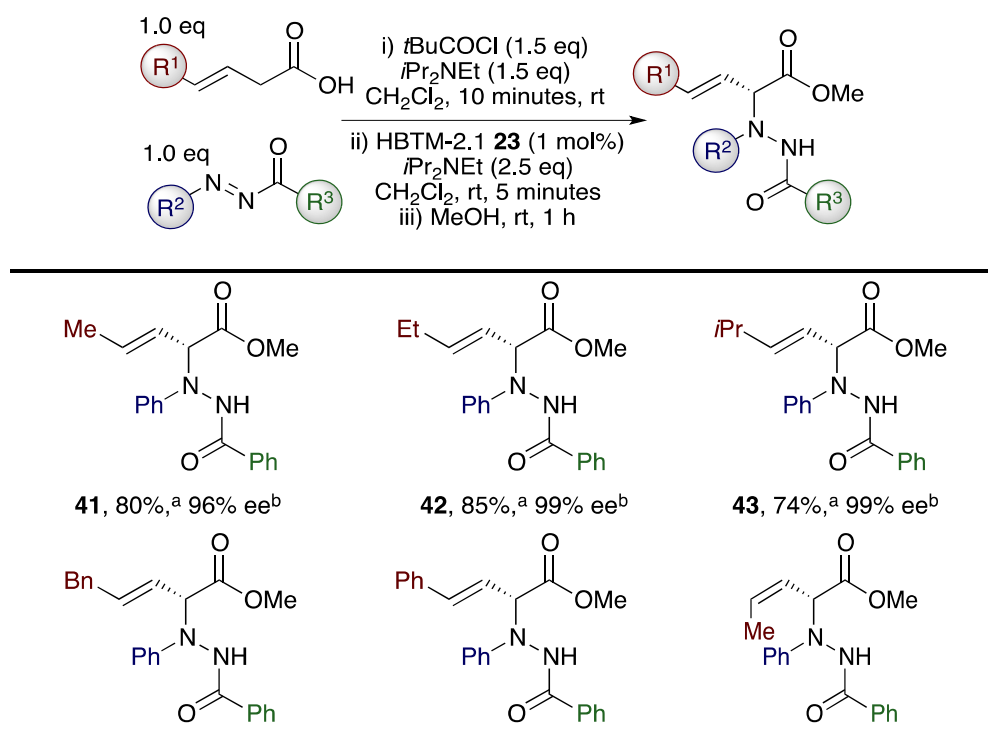

$44,77 \%,^{\text {a }} 99 \%$ ee $^{\text {b }}$
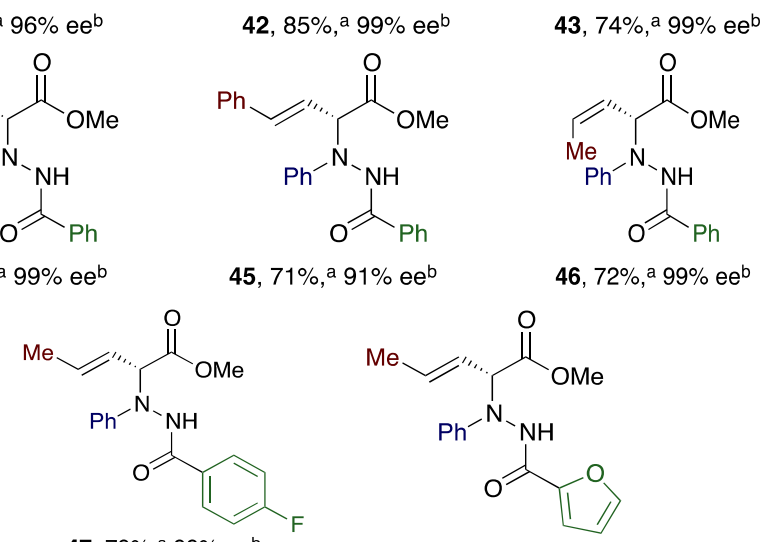

$91 \% \mathrm{ee}^{\mathrm{b}}$

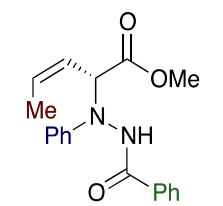

$46,72 \%,{ }^{a} 99 \% e^{b}$

$47,79 \%$, a $99 \%$ ee

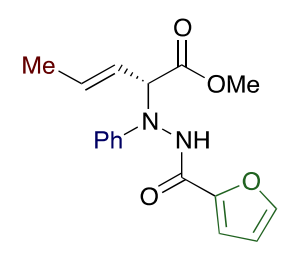

48, $87 \%$, a $99 \%$ ee

Table 5: Formal [4+2] cycloaddition/ring opening with $N$-aryl- $N$-aroyldiazenes; ${ }^{\text {a }}$ Isolated yield. ${ }^{\text {b }}$ Determined by HPLC analysis. 
Having developed a highly enantioselective route to hydrazides 41-48, their potential for further elaboration through functionalization of the olefin was probed. Treatment of hydrazide 41 under Upjohn dihydroxylation conditions, followed by acid catalysed cyclisation, gave a 70:30 mixture of separable diastereomeric 5-membered lactones 49 and $\mathbf{5 0}$ in 85\% combined yield, both in 99\% ee (Scheme 5). ${ }^{36}$ These interesting aza-sugar derivatives structurally resemble the cyclized form of $(+)$-polyoxamic acid, indicating their potential biological significance.

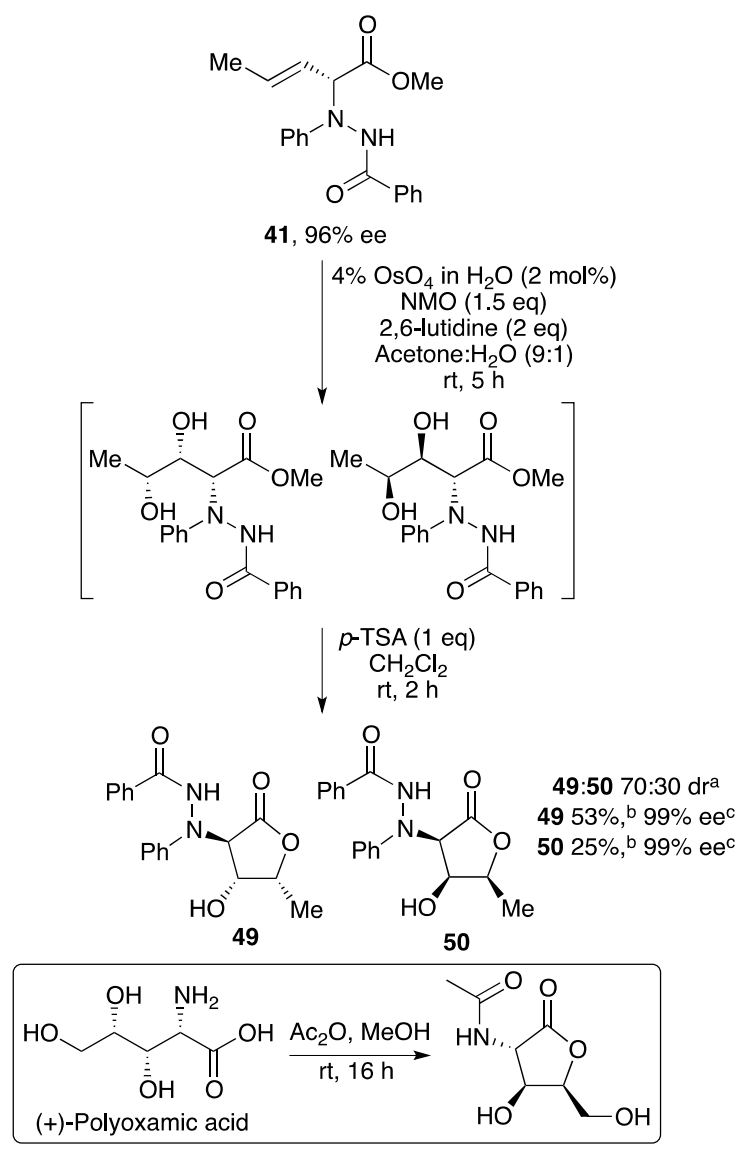

Scheme 5: Conversion of hydrazide 41 to lactones 49 and 50; ${ }^{a}$ Determined by ${ }^{1} \mathrm{H}$ NMR spectroscopic analysis of the crude reaction mixture; ${ }^{\mathrm{b}}$ Isolated yield ( $\left.>98: 2 \mathrm{dr}\right){ }^{\mathrm{c}}$ Determined by HPLC analysis.

We propose that the catalytic cycle for these transformations proceeds via initial $N$-acylation of HBTM-2.1 23 with the pre-formed mixed anhydride to form the corresponding acyl ammonium ion. $\alpha$-Deprotonation generates the $(Z, E)$-enolate (from the $(E)$-alkenoic acid), which undergoes stereoselective Michael addition via $\alpha$-functionalization with electron deficient $4 \pi$ Michael acceptors, followed by intramolecular 
cyclisation, to generate the corresponding heterocyclic species (Figure 1). The sense of stereoinduction in these transformations is consistent with our previous rationale. ${ }^{16 a, c}$ We tentatively assign the origin of the observed $\alpha$-functionalization in these processes to preferential reaction via the assumed $s$-trans $(Z, E)$ dienolate conformation $\mathbf{5 1}$, in preference to the $s$-cis $(Z, E)$-dienolate conformation $\mathbf{5 2}$ that is presumably necessary to participate in $\gamma$-functionalization.

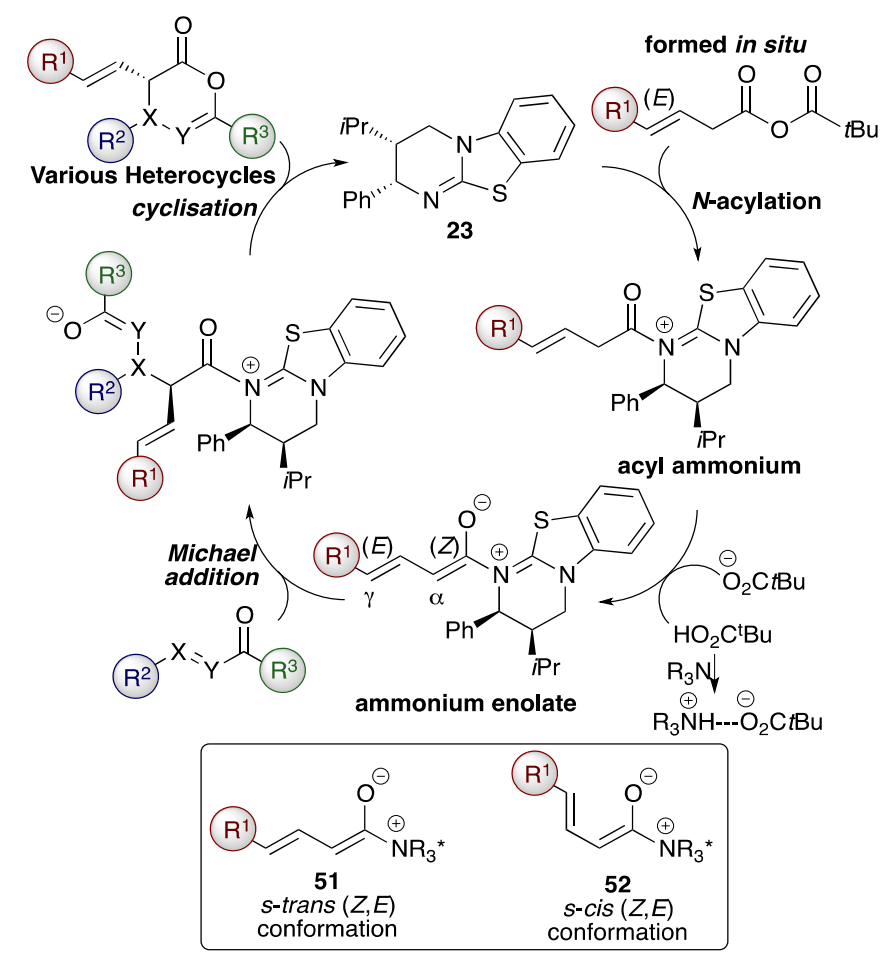

Figure 1: Proposed mechanism of asymmetric heterocycle formation.

Conclusion: Isothiourea-mediated functionalization of 3-alkenoic acids occurs regioselectively, giving products derived from $\alpha$-functionalization of an intermediate ammonium enolate in a range of formal $[2+2]$ and $[4+2]$ cycloadditions. Formal [2+2] cycloadditions with $N$-tosyl aldimines proceed readily using HBTM-2.1 (10 mol\%) with moderate diastereocontrol (up to 87:13 dr) and excellent enantiocontrol (up to $>99 \%$ ee). Formal [4+2] cycloadditions with either 4-aryl-trifluoromethylenones or $N$-aryl- $N$ aroyldiazenes are also catalyzed by HBTM-2.1 (1-5 mol\%), with products obtained in high diastereo- and enantiocontrol (up to 95:5 dr, up to 99\% ee). The simple, two-step elaboration of stereodefined hydrazides into aza-sugar analogues without erosion of enantiopurity has also been demonstrated. Current research 
from this laboratory is directed toward developing alternative applications of isothioureas in asymmetric catalysis.

\section{Experimental:}

\section{General Information}

Reactions involving moisture sensitive reagents were carried out under an argon atmosphere using standard vacuum line techniques in addition to dry solvents. In these cases, all glassware used was flame dried and cooled under vacuum.

For moisture sensitive reactions, solvents (THF, $\mathrm{CH}_{2} \mathrm{Cl}_{2}$, toluene, hexane and $\mathrm{Et}_{2} \mathrm{O}$ ) were obtained anhydrous and purified by an alumina column. Petrol is defined as petroleum ether $40-60{ }^{\circ} \mathrm{C}$. All other solvents and commercial reagents were used as supplied without further purification unless stated otherwise. Room temperature (rt) refers to $20-25^{\circ} \mathrm{C}$, with temperatures of 0 and $-78{ }^{\circ} \mathrm{C}$ obtained using ice/water and $\mathrm{CO} 2(\mathrm{~s}) /$ acetone baths, respectively. $1 \mathrm{H}$ NMR spectra were acquired at 300, 400, or 500 $\mathrm{MHz}, 13 \mathrm{C}\{1 \mathrm{H}\} \mathrm{NMR}$ spectra were acquired at 75, 100, or $125 \mathrm{MHz}$, and $19 \mathrm{~F}\{1 \mathrm{H}\} \mathrm{NMR}$ spectra were acquired at 282, 376, or $471 \mathrm{MHz}$. Chemical shifts are quoted in parts per million (ppm) relative to the residual solvent peak. Coupling constants, J, are quoted in Hertz (Hz). NMR peak assignments were confirmed using 2D 1H correlated spectroscopy (COSY), 2D 1H nuclear Overhauser effect spectroscopy (NOESY), 2D 1H-13C heteronuclear multiple-bond correlation spectroscopy (HMBC), and 2D 1H-13C heteronuclear single quantum coherence (HSQC) where necessary. Infrared spectra were recorded as thin films using an attenuated total reflectance (ATR) accessory. Mass spectrometry ( $\mathrm{m} / \mathrm{z}$ ) data was acquired using electrospray ionization (ESI), electron impact (EI), chemical ionization (CI), atmospheric solids analysis probe (ASAP), atmospheric pressure chemical ionization (APCI), or nanospray ionization (NSI) using a time of flight (TOF) mass analyzer. Optical rotations were recorded with a path length of $1 \mathrm{dm}$ and concentrations, c, are quoted in g/100 mL. All chiral high-performance liquid chromatography (HPLC) traces were compared with an authentic racemic trace. Racemic compounds were prepared using general procedure A, employing either DHPB 17 or $( \pm)$-HBTM-2.1 23 as catalyst. 


\section{Isothiourea catalysts used}

DHPB 17, HBTM-2.1 ( \pm )-23 and HBTM-2.1 (2S,3R)-23 were made to literature procedures. ${ }^{16 \mathrm{~d}}$

\section{$N$-tosyl aldimines used}

4-methyl-N-[(1E)-phenylmethylidene]benzene-1-sulfonamide

19,

4-methyl-N-[(1E)-4-

(bromophenyl)methylidene]benzene-1-sulfonamide

25 ,

4-methyl-N-[(1E)-4-

(methoxyphenyl)methylidene]benzene-1-sulfonamide

53 ,

4-methyl-N-[(1E)-4-

(trifluoromethylphenyl)methylidene]benzene-1-sulfonamide

54, N-[(1E)-furan-2-ylmethylidene]-4methylbenzene-1-sulfonamide $\mathbf{5 5}$ and 4-methyl-N-[(1E)-naphthalen-2-ylmethylidene]benzene-1sulfonamide $\mathbf{5 6}$ were made according to literature procedures. ${ }^{37}$

\section{Trifluoromethyl enones used}

(E)-1,1,1-trifluoro-4-phenyl-3-buten-2-one 57, (E)-1,1,1-trifluoro-4-(4-bromophenyl)-3-buten-2-one 58 and (E)-1,1,1-trifluoro-4-(2-thienyl)-3-buten-2-one 59 were made according to literature procedures. ${ }^{16 \mathrm{~d}}$

\section{$N$-aryl- $N$-aroyldiazenes used}

$(\mathrm{N} E)-\mathrm{N}-($ phenylimino)benzamide $\quad \mathbf{6 0}, \quad(\mathrm{N} E)$-4-fluoro-N-(phenylimino)benzamide $\quad \mathbf{6 1}$ and $(\mathrm{N} E)-\mathrm{N}-$ (phenylimino)furan-2-carboxamide 62 were made according to literature procedures. ${ }^{16 \mathrm{c}}$

\section{Carboxylic acids used}

(E)-4-phenylbut-3-enoic acid 16, (E)-pent-3-enoic acid 24 and (E)-hex-3-enoic acid 81 were used as purchased.

\section{3-methylbut-3-enoic acid 14}

Following a literature procedure, ${ }^{38}$ to a solution of 3-methylbut-3-en-1-ol (2.00 mL, $\left.19.8 \mathrm{mmol}\right)$ in acetone $(100 \mathrm{~mL})$ at $0{ }^{\circ} \mathrm{C}$ was added $2.68 \mathrm{M}$ Jones' reagent $(10.4 \mathrm{~mL}, 27.7 \mathrm{mmol})$ and the reaction mixture was stirred at $0{ }^{\circ} \mathrm{C}$ for $1 \mathrm{~h}$. The reaction mixture was washed with $2 \mathrm{M} \mathrm{NaOH}$ and then the aqueous layer acidified with conc $\mathrm{HCl}$ and extracted with $\mathrm{Et}_{2} \mathrm{O}$ (x 3). The combined organic extracts were dried $\left(\mathrm{MgSO}_{4}\right)$, filtered and concentrated in vacuo. The residual oil was purified by distillation to give acid 3- 
methylbut-3-enoic acid 14 as a colourless oil (1.50 g, 76\%); bp $88-90{ }^{\circ} \mathrm{C}(20 \mathrm{mmHg})$; $\left\{\right.$ lit. ${ }^{38}$ bp $67-70{ }^{\circ} \mathrm{C}$ $(10 \mathrm{mmHg})\} ; \delta_{\mathrm{H}}\left(500 \mathrm{MHz}, \mathrm{CDCl}_{3}\right) 1.87\left(3 \mathrm{H}, \mathrm{s}, \mathrm{CH}_{3}\right), 3.11\left(2 \mathrm{H}, \mathrm{s}, \mathrm{CH}_{2}\right), 4.92(1 \mathrm{H}, \mathrm{s},=\mathrm{CHH}), 4.99(1 \mathrm{H}$, $\mathrm{s},=\mathrm{CH} H)$. Data are in accordance with the literature. ${ }^{38}$

\section{ethyl 2-cyclopentylideneacetate 63}

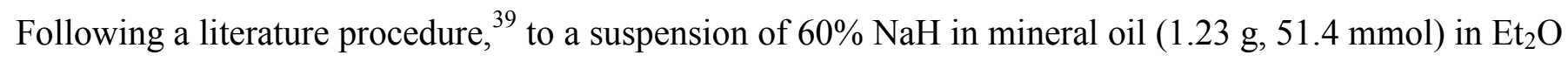
$(120 \mathrm{~mL})$ at $0{ }^{\circ} \mathrm{C}$ was added ethyl 2-(diethoxyphosphoryl)acetate $(10.2 \mathrm{~mL}, 51.4 \mathrm{mmol})$ and the reaction mixture was stirred for 5 minutes at $0{ }^{\circ} \mathrm{C}$. A solution of cyclopentanone $(4.42 \mathrm{~mL}, 50.0 \mathrm{mmol})$ in $\mathrm{Et}_{2} \mathrm{O}(10$ $\mathrm{mL}$ ) was added and the reaction mixture was allowed to stir at $\mathrm{rt}$ for $4 \mathrm{~h}$. The reaction mixture was diluted with water and extracted with $\mathrm{Et}_{2} \mathrm{O}$ (x 3). The combined organic extracts were dried $\left(\mathrm{MgSO}_{4}\right)$, filtered and concentrated in vacuo. Chromatographic purification (eluent $\mathrm{Et}_{2} \mathrm{O}$ :petrol 10:90) gave ethyl 2cyclopentylideneacetate $\mathbf{6 3}$ as a colourless oil $(7.00 \mathrm{~g}, 91 \%) ; \delta_{\mathrm{H}}\left(500 \mathrm{MHz}, \mathrm{CDCl}_{3}\right) 1.30(3 \mathrm{H}, \mathrm{t}, J 7.1$, $\left.\mathrm{CH}_{3}\right), 1.68\left(2 \mathrm{H}\right.$, quintet, $J$ 6.8, $\left.\mathrm{CH}_{2}\right), 1.77\left(2 \mathrm{H}\right.$, quintet, $J$ 7.0, $\left.\mathrm{CH}_{2}\right), 2.44-2.47\left(2 \mathrm{H}, \mathrm{m}, \mathrm{CH} \mathrm{C}_{2}=\right), 2.78-2.81$ $\left(2 \mathrm{H}, \mathrm{m}, \mathrm{CH}_{2} \mathrm{C}=\right), 4.17\left(2 \mathrm{H}, \mathrm{q}, J 7.1, \mathrm{CH}_{2} \mathrm{CH}_{3}\right), 5.82(1 \mathrm{H}$, quintet, $J 2.2,=\mathrm{CH})$. Data are in accordance with the literature. ${ }^{39}$

\section{ethyl 2-(cyclopent-1-en-1-yl)acetate 64}

Following a literature procedure, ${ }^{40}$ To a solution of DIPA $(5.82 \mathrm{~mL}, 41.2 \mathrm{mmol})$ in $\mathrm{THF}(80 \mathrm{~mL})$ at $0{ }^{\circ} \mathrm{C}$ was added 2.5 $\mathrm{M} n$-BuLi $(16.5 \mathrm{~mL}, 41.2 \mathrm{mmol})$ and the reaction mixture was stirred at that temperature for 30 minutes. The reaction mixture was cooled to $-78{ }^{\circ} \mathrm{C}$ and a solution of ethyl 2-(cyclopent-1-en-1yl)acetate 63 (5.88 g, $38.2 \mathrm{mmol})$ in THF (25 mL) was added dropwise over 15 minutes before stirring for a further 20 minutes. The reaction mixture was quenched by addition of sat. aq. $\mathrm{NH}_{4} \mathrm{Cl}$ and the reaction mixture was warmed to $\mathrm{rt}$ before being poured into water and extracted with $\mathrm{Et}_{2} \mathrm{O}(\mathrm{x} 3)$. The combined organic extracts were dried $\left(\mathrm{MgSO}_{4}\right)$, filtered and concentrated in vacuo to give ethyl 2-(cyclopent-1-en-1yl)acetate 64 as a light yellow oil $(5.68 \mathrm{~g}, 97 \%) ; \delta_{\mathrm{H}}\left(500 \mathrm{MHz}, \mathrm{CDCl}_{3}\right) 1.29\left(3 \mathrm{H}, \mathrm{t}, J 7.1, \mathrm{CH}_{3}\right), 1.93(2 \mathrm{H}$, 
quintet, $J$ 7.5, $\left.\mathrm{CH}_{2}\right), 2.33-2.38\left(4 \mathrm{H}, \mathrm{m}, \mathrm{CH}_{2}\right.$ and $\left.\mathrm{CH}_{2}\right), 3.14\left(2 \mathrm{H}, \mathrm{s}, \mathrm{CH}_{2} \mathrm{CO}_{2} \mathrm{Et}\right), 4.17(2 \mathrm{H}, \mathrm{q}, J$ 7.1, $\left.\mathrm{CH}_{2} \mathrm{CH}_{3}\right), 5.55-5.57(1 \mathrm{H}, \mathrm{m},=\mathrm{CH})$. Data are in accordance with the literature. ${ }^{40}$

\section{2-(cyclopent-1-en-1-yl)acetic acid 15}

Following a literature procedure, ${ }^{41}$ a solution of ethyl 2-(cyclopent-1-en-1-yl)acetate 64 (4.15 g, 27.0 $\mathrm{mmol})$ in $0.5 \mathrm{M} \mathrm{KOH}(80.8 \mathrm{~mL}, 40.4 \mathrm{mmol})$ was heated at reflux for $16 \mathrm{~h}$. Once cooled to rt the reaction mixture was extracted with $\mathrm{Et}_{2} \mathrm{O}$ (x 3). The combined organic extracts were dried $\left(\mathrm{MgSO}_{4}\right)$, filtered and concentrated in vacuo. Recrystallisation from petrol gave 2-(cyclopent-1-en-1-yl)acetic acid $\mathbf{1 5}$ as a white solid (2.74 g, 59\%); mp 44-46 ${ }^{\circ} \mathrm{C} ;\left\{\right.$ lit. $\left.{ }^{41} \mathrm{mp} 48-51{ }^{\circ} \mathrm{C}\right\} ; \delta_{\mathrm{H}}\left(300 \mathrm{MHz}, \mathrm{CDCl}_{3}\right)$ 1.90-2.00 (2H, m, $\left.\mathrm{CH}_{2}\right)$, 2.37-2.42 (4H, m, $\mathrm{CH}_{2}$ and $\left.\mathrm{CH}_{2}\right), 3.21\left(2 \mathrm{H}, \mathrm{s}, \mathrm{CH}_{2} \mathrm{CO}_{2} \mathrm{H}\right), 5.63(1 \mathrm{H}, \mathrm{m},=\mathrm{CH})$. Data are in accordance with the literature. ${ }^{41}$

\section{(E)-5-methylhex-3-enoic acid 65}

Following a literature procedure, ${ }^{42}$ a solution of piperidine $(39.5 \mu \mathrm{L}, 0.40 \mathrm{mmol})$ and acetic acid $(22.9 \mu \mathrm{L}$, $0.40 \mathrm{mmol})$ in DMSO (1 mL) was stirred at $\mathrm{rt}$ for 5 minutes after which time a solution of malonic acid (4.16 g, $40.0 \mathrm{mmol})$ and isovaleraldehyde (4.29 $\mathrm{mL}, 40.0 \mathrm{mmol})$ in DMSO (20 $\mathrm{mL})$ was added. The reaction mixture was stirred at $\mathrm{rt}$ for 20 minutes and then at $100{ }^{\circ} \mathrm{C}$ for $16 \mathrm{~h}$. Once cooled to rt, the reaction mixture was diluted with $\mathrm{H}_{2} \mathrm{O}$ and extracted with $\mathrm{Et}_{2} \mathrm{O}$ (x 3). The combined organic fractions were washed with $\mathrm{H}_{2} \mathrm{O}$, dried $\left(\mathrm{MgSO}_{4}\right)$, filtered and concentrated in vacuo. Chromatographic purification (eluent $\mathrm{Et}_{2} \mathrm{O}$ :petrol 30:70) gave (E)-5-methylhex-3-enoic acid 65 as a colourless oil $(2.93 \mathrm{~g}, 57 \%) ; \delta_{\mathrm{H}}(400$ $\left.\mathrm{MHz}, \mathrm{CDCl}_{3}\right) 0.99\left(6 \mathrm{H}, \mathrm{d}, J\right.$ 6.8, $\left.\mathrm{CH}\left(\mathrm{CH}_{3}\right)_{2}\right), 2.26-2.34(1 \mathrm{H}, \mathrm{m}, \mathrm{C}(5) H), 3.07\left(2 \mathrm{H}, \mathrm{dt}, J\right.$ 6.6, 0.9, C(2)H $\left.H_{2}\right)$ $5.47(1 \mathrm{H}, \mathrm{dtd}, J 15.4,6.7,1.1, \mathrm{C}(3) H), 5.54-5.60(1 \mathrm{H}, \mathrm{m}, \mathrm{C}(4) H)$. Data are in accordance with the literature. $^{42}$

\section{(E)-5-phenylpent-3-enoic acid 66}

Following a literature procedure, ${ }^{42}$ a solution of piperidine $(39.5 \mu \mathrm{L}, 0.40 \mathrm{mmol})$ and acetic acid $(22.9 \mu \mathrm{L}$, $0.40 \mathrm{mmol})$ in DMSO (1 mL) was stirred at $\mathrm{rt}$ for 5 minutes after which time a solution of malonic acid 
(4.16 g, $40.0 \mathrm{mmol})$ and 3-phenylpropionaldehyde $(5.28 \mathrm{~mL}, 40.0 \mathrm{mmol})$ in DMSO (20 mL) was added. The reaction mixture was stirred at $\mathrm{rt}$ for 20 minutes and then at $100{ }^{\circ} \mathrm{C}$ for $16 \mathrm{~h}$. Once cooled to $\mathrm{rt}$, the reaction mixture was diluted with $\mathrm{H}_{2} \mathrm{O}$ and extracted with $\mathrm{Et}_{2} \mathrm{O}$ (x 3). The combined organic fractions were washed with $\mathrm{H}_{2} \mathrm{O}$, dried $\left(\mathrm{MgSO}_{4}\right)$, filtered and concentrated in vacuo. Chromatographic purification (eluent $\mathrm{Et}_{2} \mathrm{O}$ :petrol 25:75) gave (E)-5-phenylpent-3-enoic acid 66 as a colourless oil (4.15 g, 59\%); $\delta_{\mathrm{H}}(400$ $\left.\mathrm{MHz}, \mathrm{CDCl}_{3}\right) 3.13(2 \mathrm{H}, \mathrm{dq}, J$ 6.8, 1.1, C(5)H $), 3.40(2 \mathrm{H}, \mathrm{d}, J$ 6.7, C(2)H $), 5.63(1 \mathrm{H}, \mathrm{dtt}, J$ 15.3, 6.9, 1.4, $\mathrm{C}(3) H), 5.77(1 \mathrm{H}, \mathrm{dtt}, J 15.3,6.7,1.3, \mathrm{C}(4) H), 7.18-7.23(3 \mathrm{H}, \mathrm{m}, \operatorname{Ar}(2,6) H$ and $\operatorname{Ar}(4) H), 7.28-7.32(2 \mathrm{H}$, $\mathrm{m}, \operatorname{Ar}(3,5) H)$. Data are in accordance with the literature. ${ }^{43}$

\section{(Z)-pent-3-en-1-ol 67}

Following a literature procedure, ${ }^{44}$ lindlar's catalyst $\left(5 \%\right.$ on $\mathrm{CaCO}_{3}, \mathrm{~Pb}$ poisoned, $900 \mathrm{mg}(45 \mathrm{mg} \mathrm{Pd})$, $0.43 \mathrm{mmol})$ was degassed in a RB flask. Quinoline $(0.72 \mathrm{~mL}, 6.04 \mathrm{mmol}), \mathrm{Et}_{2} \mathrm{O}(150 \mathrm{~mL})$ and pent-3-yn1-ol (2.74 mL, $29.7 \mathrm{mmol})$ were added and a balloon of $\mathrm{H}_{2}$ gas was appended to the reaction flask. $\mathrm{H}_{2}$ gas was bubbled through the reaction mixture at $\mathrm{rt}$ for $20 \mathrm{~h}$. The reaction mixture was filtered through Celite concentrated in vacuo and the residual oil was purified by distillation to give alcohol (Z)-pent-3-en-1-ol 67 (94:6 (Z):(E)) as a colourless oil $(1.64 \mathrm{~g}, 64 \%)$; bp $140-141{ }^{\circ} \mathrm{C}(760 \mathrm{mmHg}) ;\left\{\right.$ lit. ${ }^{44}$ bp $140{ }^{\circ} \mathrm{C}(760$ $\mathrm{mmHg})\}$; Data for $(Z)$-isomer: $\delta_{\mathrm{H}}\left(500 \mathrm{MHz} \mathrm{CDCl}_{3}\right)$ 1.65-1.68 $\left(3 \mathrm{H}, \mathrm{m}, \mathrm{CH}_{3}\right), 2.32-2.37\left(2 \mathrm{H}, \mathrm{m}, \mathrm{C}(2) H_{2}\right)$, $3.66\left(2 \mathrm{H}, \mathrm{q}, J 6.2, \mathrm{C}(1) H_{2}\right), 5.37-5.43(1 \mathrm{H}, \mathrm{m}, \mathrm{C}(4) H), 5.62-5.68(1 \mathrm{H}, \mathrm{m}, \mathrm{C}(3) H)$; Selected data for $(E)-$ isomer: $\delta_{\mathrm{H}}\left(500 \mathrm{MHz}, \mathrm{CDCl}_{3}\right) 1.68-1.70\left(3 \mathrm{H}, \mathrm{m}, \mathrm{CH}_{3}\right), 2.23-2.28\left(2 \mathrm{H}, \mathrm{m}, \mathrm{C}(2) \mathrm{H}_{2}\right)$. Data are in accordance with the literature. ${ }^{44}$

\section{(Z)-pent-3-enoic acid 68}

Following a literature procedure, ${ }^{44}$ to $\mathrm{K}_{2} \mathrm{Cr}_{2} \mathrm{O}_{7}(56.1 \mathrm{mg}, 0.19 \mathrm{mmol}), \mathrm{HNO}_{3}(343 \mathrm{mg}, 3.81 \mathrm{mmol})$ and $\mathrm{NaIO}_{4}(8.97 \mathrm{~g}, 42.0 \mathrm{mmol})$ in $\mathrm{H}_{2} \mathrm{O}(25 \mathrm{~mL})$ was added a solution of (Z)-pent-3-en-1-ol 67 (1.64 g, 19.1 $\mathrm{mmol})$ in $\mathrm{MeCN}(50 \mathrm{~mL})$ at $0{ }^{\circ} \mathrm{C}$. The reaction mixture was stirred at $0{ }^{\circ} \mathrm{C}$ for $8 \mathrm{~h}$ followed by $\mathrm{rt}$ for $16 \mathrm{~h}$. The inorganic salts were filtered and washed with $\mathrm{Et}_{2} \mathrm{O} \cdot \mathrm{H}_{2} \mathrm{O}$ was added and the reaction mixture was 
extracted with $\mathrm{Et}_{2} \mathrm{O}$ (x 3). The combined organic fractions were dried $\left(\mathrm{MgSO}_{4}\right)$, filtered and concentrated in vacuo. The residual oil was purified by distillation to give $(Z)$-pent-3-enoic acid $68(94: 6(Z):(E))$ as a colourless oil (0.69 g, 36\%); bp 100-102 ${ }^{\circ} \mathrm{C}(22 \mathrm{mmHg}) ;\left\{\right.$ lit. ${ }^{44}$ bp $\left.100{ }^{\circ} \mathrm{C}(20 \mathrm{mmHg})\right\}$; Data for $(Z)$ isomer: $\delta_{\mathrm{H}}\left(500 \mathrm{MHz}, \mathrm{CDCl}_{3}\right) 1.64\left(3 \mathrm{H}, \mathrm{dt}, J 6.8,0.8, \mathrm{CH}_{3}\right), 3.14\left(2 \mathrm{H}, \mathrm{dd}, J 7.2,0.4, \mathrm{C}(2) H_{2}\right), 3.66(2 \mathrm{H}, \mathrm{q}$, $J$ 6.2, C(1) $\left.H_{2}\right), 5.56(1 \mathrm{H}, \mathrm{dtq}, J 10.7,7.1,1.8, \mathrm{C}(3) H), 5.66-5.73(1 \mathrm{H}, \mathrm{m}, \mathrm{C}(4) H)$; Selected data for $(E)-$ isomer: $\delta_{\mathrm{H}}\left(500 \mathrm{MHz}, \mathrm{CDCl}_{3}\right) 1.70\left(3 \mathrm{H}, \mathrm{dt}, J 6.3,1.3, \mathrm{CH}_{3}\right), 3.06\left(2 \mathrm{H}, \mathrm{dt}, J 6.7,1.2, \mathrm{C}(2) H_{2}\right)$. Data are in accordance with the literature. ${ }^{44}$

\section{(E)-ethyl 3-phenylbut-2-enoate 69}

Following a literature procedure ${ }^{45}$ to a suspension of $60 \% \mathrm{NaH}$ in mineral oil $(1.00 \mathrm{~g}, 41.6 \mathrm{mmol})$ in $\mathrm{THF}$ $(35 \mathrm{~mL})$ at $0{ }^{\circ} \mathrm{C}$ was added ethyl 2-(diethoxyphosphoryl)acetate $(8.26 \mathrm{~mL}, 41.6 \mathrm{mmol})$ dropwise over 30 minutes and the reaction mixture was stirred for 30 minutes at $\mathrm{rt}$. A solution of acetophenone $(4.85 \mathrm{~mL}$, $41.6 \mathrm{mmol})$ in THF $(15 \mathrm{~mL})$ was added dropwise and the reaction mixture was allowed to stir at $\mathrm{rt}$ for $4 \mathrm{~h}$. The reaction mixture was diluted with water and extracted with $\mathrm{Et}_{2} \mathrm{O}$ (x 3). The combined organic extracts were dried $\left(\mathrm{MgSO}_{4}\right)$, filtered and concentrated in vacuo. Chromatographic purification (eluent $\mathrm{Et}_{2} \mathrm{O}$ :petrol 5:95) gave (E)-ethyl 3-phenylbut-2-enoate 69 as a colourless oil $(2.35 \mathrm{~g}, 30 \%) ; \delta_{\mathrm{H}}\left(500 \mathrm{MHz}, \mathrm{CDCl}_{3}\right) 1.35$ $\left(3 \mathrm{H}, \mathrm{t}, J\right.$ 7.1, $\left.\mathrm{CH}_{2} \mathrm{CH}_{3}\right), 2.61\left(3 \mathrm{H}, \mathrm{d}, J 1.3, \mathrm{CH}_{3}\right), 4.25\left(2 \mathrm{H}, \mathrm{q}, J\right.$ 7.1, $\left.\mathrm{CH}_{2} \mathrm{CH}_{3}\right), 6.16(1 \mathrm{H}, \mathrm{q}, J 1.2,=\mathrm{CH})$, 7.38-7.42 $(3 \mathrm{H}, \mathrm{m}, \mathrm{ArH}), 7.50-7.52(2 \mathrm{H}, \mathrm{m}, \mathrm{ArH})$. Data are in accordance with the literature. ${ }^{45}$

\section{(E)-3-phenylbut-2-enoic acid 70}

Following a literature procedure, ${ }^{41}$ a solution of $(E)$-ethyl 3-phenylbut-2-enoate 69 (2.35 g, $\left.12.4 \mathrm{mmol}\right)$ in $0.5 \mathrm{M} \mathrm{KOH}(37.1 \mathrm{~mL}, 18.6 \mathrm{mmol})$ was heated at reflux for $16 \mathrm{~h}$. Once cooled to rt the reaction mixture was extracted with $\mathrm{Et}_{2} \mathrm{O}$ (x 3). The reaction mixture was treated with $1 \mathrm{M} \mathrm{H}_{2} \mathrm{SO}_{4}$ until acidic and extracted with $\mathrm{Et}_{2} \mathrm{O}$ (x 3). The combined organic extracts were dried $\left(\mathrm{MgSO}_{4}\right)$, filtered and concentrated in vacuo. Recrystallisation from $\mathrm{Et}_{2} \mathrm{O}$ gave $(E)$-3-phenylbut-2-enoic acid 70 as a white solid (1.39 g, 70\%); mp 94- 
$96{ }^{\circ} \mathrm{C} ;\left\{\right.$ lit. $\left.^{46} \mathrm{mp} 95-97{ }^{\circ} \mathrm{C}\right\} ; \delta_{\mathrm{H}}\left(500 \mathrm{MHz}, \mathrm{CDCl}_{3}\right) 2.64\left(3 \mathrm{H}, \mathrm{d}, J 1.2, \mathrm{CH}_{3}\right), 6.21(1 \mathrm{H}, \mathrm{q}, J 1.2,=\mathrm{CH})$, 7.41-7.44 (3H, m, ArH), 7.51-7.54 (2H, m, ArH). Data are in accordance with the literature. ${ }^{47}$

\section{(E)-ethyl 3,4-diphenylbut-2-enoate 71}

Following a literature procedure, ${ }^{45}$ to a suspension of $60 \% \mathrm{NaH}$ in mineral oil $(2.04 \mathrm{~g}, 51.0 \mathrm{mmol})$ in $\mathrm{THF}$ $(50 \mathrm{~mL})$ at $0{ }^{\circ} \mathrm{C}$ was added ethyl 2-(diethoxyphosphoryl)acetate $(10.1 \mathrm{~mL}, 51.0 \mathrm{mmol})$ dropwise over 30 minutes and the reaction mixture was stirred for 30 minutes at rt. A solution of 1,2-diphenylethanone (10 g, $51.0 \mathrm{mmol})$ in THF $(20 \mathrm{~mL})$ was added dropwise and the reaction mixture was allowed to stir at $\mathrm{rt}$ for 4 h. The reaction mixture was diluted with water and extracted with $\mathrm{Et}_{2} \mathrm{O}$ (x 3). The combined organic extracts were dried $\left(\mathrm{MgSO}_{4}\right)$, filtered and concentrated in vacuo. Chromatographic purification (eluent $\mathrm{Et}_{2} \mathrm{O}$ :petrol 5:95) gave (E)-ethyl 3,4-diphenylbut-2-enoate 71 as a colourless oil $(2.35 \mathrm{~g}, 17 \%) ; \delta_{\mathrm{H}}(300$ $\left.\mathrm{MHz}, \mathrm{CDCl}_{3}\right) 1.35\left(3 \mathrm{H}, \mathrm{t}, J\right.$ 7.1, $\left.\mathrm{CH}_{2} \mathrm{CH}_{3}\right), 4.27\left(2 \mathrm{H}, \mathrm{q}, J\right.$ 7.1, $\left.\mathrm{CH}_{2} \mathrm{CH}_{3}\right), 4.55\left(2 \mathrm{H}, \mathrm{s}, \mathrm{CH}_{2} \mathrm{Ph}\right), 6.29(1 \mathrm{H}, \mathrm{d}$, $=\mathrm{CH}), 7.14-7.27(5 \mathrm{H}, \mathrm{m}, \mathrm{ArH}), 7.33-7.36(3 \mathrm{H}, \mathrm{m}, \mathrm{ArH}), 7.42-7.48(2 \mathrm{H}, \mathrm{m}, \mathrm{ArH})$. Data are in accordance with the literature. ${ }^{48}$

\section{(E)-3,4-diphenylbut-2-enoic acid 72}

Following a literature procedure, ${ }^{41}$ a solution of (E)-ethyl 3,4-diphenylbut-2-enoate 71 (2.35 g, 8.84 $\mathrm{mmol})$ in $0.5 \mathrm{M} \mathrm{KOH}(26.8 \mathrm{~mL}, 13.3 \mathrm{mmol})$ was heated at reflux for $16 \mathrm{~h}$. Once cooled to rt the reaction mixture was extracted with $\mathrm{Et}_{2} \mathrm{O}(\mathrm{x} 3)$. The reaction mixture was treated with $1 \mathrm{M} \mathrm{H}_{2} \mathrm{SO}_{4}$ until acidic and extracted with $\mathrm{Et}_{2} \mathrm{O}(\mathrm{x} 3)$. The combined organic extracts were dried $\left(\mathrm{MgSO}_{4}\right)$, filtered and concentrated in vacuo. Chromatographic purification (eluent $\mathrm{Et}_{2} \mathrm{O}$ :petrol 25:75) gave (E)-3,4-diphenylbut-2-enoic acid 72 as a white solid $(210 \mathrm{mg}, 10 \%) ; \mathrm{mp} 122-124{ }^{\circ} \mathrm{C} ;\left\{\right.$ lit. $\left.{ }^{49} \mathrm{mp} 138-139{ }^{\circ} \mathrm{C}\right\} ; \delta_{\mathrm{H}}\left(300 \mathrm{MHz}, \mathrm{CDCl}_{3}\right) 4.59$ (2H, s, $\left.\mathrm{CH}_{2} \mathrm{Ph}\right), 6.34(1 \mathrm{H}, \mathrm{s},=\mathrm{CH}), 7.18-7.29(5 \mathrm{H}, \mathrm{m}, \mathrm{ArH}), 7.34-7.39(3 \mathrm{H}, \mathrm{m}, \mathrm{ArH}), 7.46-7.49(2 \mathrm{H}, \mathrm{m}$, $\operatorname{Ar} H)$. Data are in accordance with the literature. ${ }^{49}$

General procedure A: Isothiourea catalysed intermolecular reactions. 
To a solution of acid (1-2 equiv.) in $\mathrm{CH}_{2} \mathrm{Cl}_{2}\left(\sim 1 \mathrm{~mL}\right.$ per $0.2 \mathrm{mmol}$ of acid) were added $i \mathrm{Pr}_{2} \mathrm{NEt}$ (1.5 equiv. based upon acid) and activating agent (1.5 equiv. based upon acid) at rt. The reaction mixture was allowed to stir at $\mathrm{rt}$ for 10 minutes. The requisite isothiourea (1-10 mol\%), Michael acceptor/ketone/imine (1 equiv.) and $i \operatorname{Pr}_{2} \mathrm{NEt}$ (2.5 equiv.) were then added at the required temperature. The reaction mixture was stirred at the required temperature until complete by TLC. The reaction mixture was subsequently quenched by addition of $\mathrm{HCl}\left(1 \mathrm{M}\right.$ in $\left.\mathrm{H}_{2} \mathrm{O}\right)$. The reaction mixture was poured into $\mathrm{H}_{2} \mathrm{O}$ and extracted with $\mathrm{CH}_{2} \mathrm{Cl}_{2}$ (x 3). The combined organics were dried $\left(\mathrm{MgSO}_{4}\right)$, filtered and concentrated in vacuo to give the crude reaction mixture.

\section{$(3 S, 4 S)$-4-phenyl-3-[(E)-2-phenylethynyl]-4-(trifluoromethyl)oxetan-2-one 21 and (3S,4R)-4-phenyl-}

\section{3-[(E)-2-phenylethynyl]-4-(trifluoromethyl)oxetan-2-one 73}

Following general procedure A, (E)-4-phenylbut-3-enoic acid $16(259 \mathrm{mg}, 1.60 \mathrm{mmol}), i \mathrm{Pr}_{2} \mathrm{NEt}(0.42 \mathrm{~mL}$, $2.40 \mathrm{mmol})$ and pivaloyl chloride $(296 \mu \mathrm{L}, 2.40 \mathrm{mmol})$ in $\mathrm{CH}_{2} \mathrm{Cl}_{2}(10 \mathrm{~mL})$, DHPB 17 (15.2 $\mathrm{mg}, 0.08$ mmol, $10 \mathrm{~mol} \%)$, 2,2,2-trifluoro-1-phenylethan-1-one $18(109 \mu \mathrm{L}, 0.80 \mathrm{mmol})$ and $i \operatorname{Pr}_{2} \mathrm{NEt}(0.35 \mathrm{ml}, 2.00$ mmol) for $1.5 \mathrm{~h}$ at $\mathrm{rt}$ gave crude lactones 21 and $\mathbf{7 3}$ (60:40 dr). Chromatographic purification (eluent $\mathrm{Et}_{2} \mathrm{O}$ :petrol 2.5:97.5) gave lactone $\mathbf{2 1}(>98: 2 \mathrm{dr})$ as a white solid (110 $\left.\mathrm{mg}, 43 \%\right)$ and lactone $\mathbf{7 3}(>98: 2 \mathrm{dr})$ as a white solid (73.4 mg, 29\%).

Following general procedure A, (E)-4-phenylbut-3-enoic acid $16(259 \mathrm{mg}, 1.60 \mathrm{mmol}), i \mathrm{Pr}_{2} \mathrm{NEt}(0.42 \mathrm{~mL}$, $2.40 \mathrm{mmol})$ and pivaloyl chloride $(296 \mu \mathrm{L}, 2.40 \mathrm{mmol})$ in $\mathrm{CH}_{2} \mathrm{Cl}_{2}(10 \mathrm{~mL})$, HBTM-2.1 (2S,3R)-23 (24.6 mg, $0.08 \mathrm{mmol}, 10 \mathrm{~mol} \%)$, 2,2,2-trifluoro-1-phenylethan-1-one $18(109 \mu \mathrm{L}, 0.80 \mathrm{mmol})$ and $i \operatorname{Pr}_{2} \mathrm{NEt}(0.35$ $\mathrm{ml}, 2.00 \mathrm{mmol})$ for $1.5 \mathrm{~h}$ at $-78{ }^{\circ} \mathrm{C}$ gave crude lactones $(3 S, 4 S)-\mathbf{2 1}$ and $(3 R, 4 R)-73(65: 35 \mathrm{dr})$. Chromatographic purification (eluent $\mathrm{Et}_{2} \mathrm{O}$ :petrol 2.5:97.5) gave lactone $(3 S, 4 S)-21(>98: 2 \mathrm{dr})$ as a white solid (98.3 mg, 39\%) and lactone $(3 R, 4 R)-\mathbf{7 3}(>98: 2 \mathrm{dr})$ as a white solid (52.6 $\mathrm{mg}, 21 \%)$ :

Data for lactone $(3 S, 4 S)-21: \mathrm{mp} 66-67{ }^{\circ} \mathrm{C} ;[\alpha]_{D}^{20}-14.8\left(c\right.$ 0.5, $\left.\mathrm{CH}_{2} \mathrm{Cl}_{2}\right)$; Chiral HPLC Chiralcel OD-H

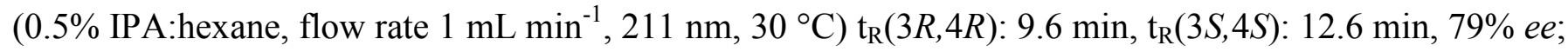


$v_{\max }(\mathrm{ATR}) / \mathrm{cm}^{-1}$ 3080, $3030(\mathrm{C}-\mathrm{H}), 1847(\mathrm{C}=\mathrm{O}), 1698 ; \delta_{\mathrm{H}}\left(400 \mathrm{MHz}, \mathrm{CDCl}_{3}\right)$ 4.95-4.98 $(1 \mathrm{H}, \mathrm{m}, \mathrm{C}(3) H)$, $5.64(1 \mathrm{H}, \mathrm{dd}, J$ 15.7, 9.4, $\mathrm{PhCH}=\mathrm{CH}), 6.71(1 \mathrm{H}, \mathrm{d}, J$ 15.7, $\mathrm{PhCH} H \mathrm{CH}), 7.20-7.23$ (2H, m, ArH), 7.27-7.31 (3H, m, ArH), 7.46-7.49 (5H, m, ArH); $\delta_{\mathrm{C}}\left(100 \mathrm{MHz}, \mathrm{CDCl}_{3}\right) 60.9(C(3)), 79.5(\mathrm{q}, J 32.8, C(4)), 116.1$ $(\mathrm{PhCH}=C H), 123.6\left(\mathrm{q}, J 280, C \mathrm{~F}_{3}\right), 126.9(A r C), 127.3(\operatorname{ArC}), 128.8(\operatorname{ArC}), 128.8(\operatorname{ArC}), 128.9(4 \mathrm{ry} A r C)$, $129.0(A r C), 130.1(A r C), 135.3\left(\mathrm{C}(4) A r C(1), 138.4(\mathrm{PhCH}=\mathrm{CH}), 165.9(C(2)=\mathrm{O}) ; \delta_{\mathrm{F}}\left(376 \mathrm{MHz}, \mathrm{CDCl}_{3}\right)\right.$ $-78.7\left(\mathrm{CF}_{3}\right) ; m / z\left(\mathrm{APCI}^{+}\right) 319\left([\mathrm{M}+\mathrm{H}]^{+}, 100 \%\right) ; \mathrm{HRMS}\left(\mathrm{APCI}^{+}\right) \mathrm{C}_{18} \mathrm{H}_{14} \mathrm{~F}_{3} \mathrm{O}_{2}^{+}\left([\mathrm{M}+\mathrm{H}]^{+}\right)$requires 319.0940; found $319.0940(-0.1 \mathrm{ppm})$.

Data for lactone $(3 S, 4 R)-73: \mathrm{mp} 110-112^{\circ} \mathrm{C} ;[\alpha]_{D}^{20}-93.0\left(c 0.5, \mathrm{CH}_{2} \mathrm{Cl}_{2}\right)$; Chiral HPLC Chiralcel OD-H $\left(2 \%\right.$ IPA:hexane, flow rate $\left.1 \mathrm{~mL} \mathrm{~min}^{-1}, 211 \mathrm{~nm}, 30^{\circ} \mathrm{C}\right) \mathrm{t}_{\mathrm{R}}(3 R, 4 S): 13.1 \mathrm{~min}, \mathrm{t}_{\mathrm{R}}(3 S, 4 R): 14.9 \mathrm{~min}, 77 \%$ ee; $v_{\max }(\mathrm{ATR}) / \mathrm{cm}^{-1}$ 3080, $2944(\mathrm{C}-\mathrm{H}), 1834(\mathrm{C}=\mathrm{O}), 1692 ; \delta_{\mathrm{H}}\left(400 \mathrm{MHz}, \mathrm{CDCl}_{3}\right) 4.77(1 \mathrm{H}, \mathrm{d}, J$ 8.7, C(3)H), 6.38-6.47 (1H, m, PhCH=CH), 6.83-6.88 (1H, m, PhCH=CH), 7.34-7.50 (9H, m, ArH); $\delta_{\mathrm{C}}(100 \mathrm{MHz}$, $\left.\mathrm{CDCl}_{3}\right) 64.9(C(3)), 79.6(\mathrm{q}, J 30.1, C(4)), 115.1(\mathrm{PhCH}=C \mathrm{H}), 123.3\left(\mathrm{q}, J 281, C \mathrm{~F}_{3}\right), 126.3(A r C), 127.1$ (ArC), $128.9(A r C), 128.9(A r C), 129.1(A r C), 130.2(A r C), 132.9$ (4ry $A r C), 135.4(\mathrm{C}(4) A r C(1)), 139.0$ $(\mathrm{PhCH}=\mathrm{CH}), 165.9(C(2)=\mathrm{O}) ; \delta_{\mathrm{F}}\left(376 \mathrm{MHz}, \mathrm{CDCl}_{3}\right)-74.2\left(\mathrm{CF}_{3}\right) ; \mathrm{m} / z\left(\mathrm{APCI}^{+}\right) 319\left([\mathrm{M}+\mathrm{H}]^{+}, 100 \%\right)$ HRMS $\left(\mathrm{APCI}^{+}\right) \mathrm{C}_{18} \mathrm{H}_{14} \mathrm{~F}_{3} \mathrm{O}_{2}^{+}\left([\mathrm{M}+\mathrm{H}]^{+}\right)$requires 319.0940; found $319.0941(+0.2 \mathrm{ppm})$.

\section{(3S,4R)-1-[(4-methylbenzene)sulfonyl]-4-phenyl-3-[(E)-2-phenylethenyl]azetidin-2-one 22}

Following general procedure A, $(E)$-4-phenylbut-3-enoic acid $16(259 \mathrm{mg}, 1.60 \mathrm{mmol}), i \operatorname{Pr}_{2} \mathrm{NEt}(0.42 \mathrm{~mL}$, $2.40 \mathrm{mmol})$ and pivaloyl chloride $(296 \mu \mathrm{L}, 2.40 \mathrm{mmol})$ in $\mathrm{CH}_{2} \mathrm{Cl}_{2}(10 \mathrm{~mL}), \mathrm{DHPB} 17$ (15.2 mg, 0.08 mmol, $10 \mathrm{~mol} \%$ ), imine 19 (207 mg, $0.80 \mathrm{mmol})(109 \mu \mathrm{L}, 0.80 \mathrm{mmol})$ and $i \operatorname{Pr}_{2} \mathrm{NEt}(0.35 \mathrm{ml}, 2.00 \mathrm{mmol})$ for $1.5 \mathrm{~h}$ at $\mathrm{rt}$ gave crude lactam 22 (83:17 dr). Chromatographic purification (eluent Et ${ }_{2} \mathrm{O}$ :petrol 20:80) gave lactam $22(>98: 2 \mathrm{dr})$ as a white solid $(219 \mathrm{mg}, 68 \%)$.

Following general procedure A, (E)-4-phenylbut-3-enoic acid 16 (260 mg, $1.60 \mathrm{mmol}), i \operatorname{Pr}_{2} \mathrm{NEt}(420 \mu \mathrm{L}$, $2.40 \mathrm{mmol})$ and pivaloyl chloride $(296 \mu \mathrm{L}, 2.40 \mathrm{mmol})$ in $\mathrm{CH}_{2} \mathrm{Cl}_{2}(10 \mathrm{~mL}), \mathrm{HBTM}-2.1(2 S, 3 R)-\mathbf{2 3}(24.6$ mg, $0.08 \mathrm{mmol}, 10 \mathrm{~mol} \%$ ), imine $19(207 \mathrm{mg}, 0.80 \mathrm{mmol})$ and $i \operatorname{Pr}_{2} \mathrm{NEt}(348 \mu \mathrm{L}, 2.00 \mathrm{mmol})$ for $1.5 \mathrm{~h}$ at $\mathrm{rt}$ 
gave crude lactam $(3 S, 4 R)-\mathbf{2 2}(85: 15 \mathrm{dr})$. Chromatographic purification (eluent $\mathrm{Et}_{2} \mathrm{O}$ :petrol 20:80) gave $\operatorname{lactam}(3 S, 4 R)-22(>98: 2 \mathrm{dr})$ as a white solid $(125 \mathrm{mg}, 39 \%) ; \mathrm{mp} 137-139{ }^{\circ} \mathrm{C} ;[\alpha]_{D}^{20}+9.8\left(c 0.5, \mathrm{CH}_{2} \mathrm{Cl}_{2}\right)$; Chiral HPLC Chiralpak AD-H (10\% IPA:hexane, flow rate $\left.1 \mathrm{~mL} \min ^{-1}, 211 \mathrm{~nm}, 30{ }^{\circ} \mathrm{C}\right) \mathrm{t}_{\mathrm{R}}(3 S, 4 R): 24.4$ $\min , \mathrm{t}_{\mathrm{R}}(3 R, 4 S): 40.9 \min , 72 \%$ ee; $v_{\max }(\mathrm{ATR}) / \mathrm{cm}^{-1}$ 3024, $2924(\mathrm{C}-\mathrm{H}), 1790(\mathrm{C}=\mathrm{O}), 1450,1359(\mathrm{~S}=\mathrm{O})$, $1165(\mathrm{~S}=\mathrm{O})$; Data for major diastereoisomer: $\delta_{\mathrm{H}}\left(300 \mathrm{MHz}, \mathrm{CDCl}_{3}\right) 2.44\left(3 \mathrm{H}, \mathrm{s}, \mathrm{CH}_{3}\right), 3.91(1 \mathrm{H}, \mathrm{ddd}, J$ 8.0, 3.3, $1.1 \mathrm{~Hz}, \mathrm{C}(3) H), 4.90(1 \mathrm{H}, \mathrm{d}, J 3.3 \mathrm{~Hz}, \mathrm{C}(4) H), 6.16(1 \mathrm{H}, \mathrm{dd}, J 15.9,8.0 \mathrm{~Hz}, \mathrm{PhCH}=\mathrm{CH}), 6.53$ $(1 \mathrm{H}, \mathrm{dd}, J 15.9,1.1 \mathrm{~Hz}, \mathrm{PhCH}=\mathrm{CH}), 7.24-7.36(12 \mathrm{H}, \mathrm{m}, \mathrm{Ar} H), 7.65-7.69\left(2 \mathrm{H}, \mathrm{m}, \mathrm{SO}_{2} \operatorname{Ar}(2,6) H\right) ; \delta_{\mathrm{C}}(100$ $\left.\mathrm{MHz}, \mathrm{CDCl}_{3}\right) 21.8\left(\mathrm{CH}_{3}\right), 62.9(\mathrm{C}(3)), 64.3(C(4)), 119.3(\mathrm{HC}=\mathrm{CHPh}), 126.6(\operatorname{ArC}), 126.8(\operatorname{ArC}), 127.7$ ( $A r C), 128.4(A r C), 128.8(A r C), 129.1(A r C), 129.1(A r C), 130.0(A r C), 135.8(\mathrm{HC}=C H P h), 135.8(4 r y$ $A r C$ ), 135.8 (4ry $A r C$ ), 135.8 (4ry $A r C), 145.4(\mathrm{C}(4) \operatorname{ArC}(1)), 165.2(C(2)=\mathrm{O}) ; \mathrm{m} / z(\mathrm{NSI}) 404\left([\mathrm{M}+\mathrm{H}]^{+}\right.$, 65\%); HRMS (NSI) $\mathrm{C}_{24} \mathrm{H}_{22} \mathrm{NO}_{3} \mathrm{~S}^{+}\left([\mathrm{M}+\mathrm{H}]^{+}\right)$requires 404.1315; found 404.1313 (-0.5 ppm).

\section{(3S,4S)-1-[(4-methylbenzene)sulfonyl]-4-phenyl-3-[(E)-2-phenylethenyl]azetidin-2-one 74}

Following general procedure A, (E)-4-phenylbut-3-enoic acid 16 (260 mg, $1.60 \mathrm{mmol}), i \operatorname{Pr}_{2} \mathrm{NEt}(420 \mu \mathrm{L}$, $2.40 \mathrm{mmol})$ and pivaloyl chloride $(296 \mu \mathrm{L}, 2.40 \mathrm{mmol})$ in $\mathrm{CH}_{2} \mathrm{Cl}_{2}(10 \mathrm{~mL}), \mathrm{HBTM}-2.1(2 S, 3 R)-23$ (24.6 mg, $0.08 \mathrm{mmol}, 10 \mathrm{~mol} \%)$, imine $19(207 \mathrm{mg}, 0.80 \mathrm{mmol})$ and $i \operatorname{Pr}_{2} \mathrm{NEt}(348 \mu \mathrm{L}, 2.00 \mathrm{mmol})$ for $2.5 \mathrm{~h}$ at $-78{ }^{\circ} \mathrm{C}$ gave crude lactam $(3 S, 4 S)-74(83: 17 \mathrm{dr})$. Chromatographic purification (eluent Et $2 \mathrm{O}:$ petrol 20:80) gave lactam $(3 S, 4 S)-74(>98: 2 \mathrm{dr})$ as a white solid $(136 \mathrm{mg}, 42 \%) ; \mathrm{mp} 127-129{ }^{\circ} \mathrm{C} ;[\alpha]_{D}^{20}-6.4(c 0.5$, $\left.\mathrm{CH}_{2} \mathrm{Cl}_{2}\right)$; Chiral HPLC Chiralpak AD-H (10\% IPA:hexane, flow rate $\left.1 \mathrm{~mL} \mathrm{~min}{ }^{-1}, 211 \mathrm{~nm}, 30{ }^{\circ} \mathrm{C}\right)$ $\mathrm{t}_{\mathrm{R}}(3 S, 4 S): 23.0 \mathrm{~min}, \mathrm{t}_{\mathrm{R}}(3 R, 4 R): 46.8 \mathrm{~min}, 16 \% e e ; v_{\max }(\mathrm{ATR}) / \mathrm{cm}^{-1} 3024,2924(\mathrm{C}-\mathrm{H}), 1790(\mathrm{C}=\mathrm{O}), 1450$ $1359(\mathrm{~S}=\mathrm{O}), 1165(\mathrm{~S}=\mathrm{O})$; Data for major diastereoisomer: $\delta_{\mathrm{H}}\left(500 \mathrm{MHz}, \mathrm{CDCl}_{3}\right) 2.48\left(3 \mathrm{H}, \mathrm{s}, \mathrm{CH}_{3}\right), 4.40-$ $4.42(1 \mathrm{H}, \mathrm{m}, \mathrm{C}(3) H), 5.38(1 \mathrm{H}, \mathrm{d}, J 6.7 \mathrm{~Hz}, \mathrm{C}(4) H), 5.49(1 \mathrm{H}, \mathrm{dd}, J 15.9,7.3 \mathrm{~Hz}, \mathrm{PhCH}=\mathrm{CH}), 6.61(1 \mathrm{H}, \mathrm{d}$, $J$ 15.8 Hz, $\mathrm{PhCH}=\mathrm{CH})$, 7.06-7.07 (2H, m, ArH), 7.16-7.24 (5H, m, ArH), 7.28-7.34 (5H, m, Ar- H), 7.80 $\left(2 \mathrm{H}, \mathrm{d}, J 8.4 \mathrm{~Hz}, \mathrm{SO}_{2} \mathrm{Ar}(2,6) H\right) ; \delta_{\mathrm{C}}\left(100 \mathrm{MHz}, \mathrm{CDCl}_{3}\right) 21.9\left(\mathrm{CH}_{3}\right), 58.1(C(4)), 61.8(\mathrm{C}(3)), 118.4$ $(\mathrm{HC}=\mathrm{CHPh}), 126.5(\mathrm{C}(4) \operatorname{ArC}(2,6)), 127.5(\operatorname{ArC}), 127.8(\operatorname{ArC}), 128.2(A r C), 128.6(\operatorname{ArC}), 128.6(\operatorname{ArC})$, 
$128.9(A r C), 130.0(A r C), 133.7$ (4ry $A r C), 135.8$ (4ry $A r C), 135.9(\mathrm{HC}=C H P h), 136.1$ (4ry $A r C), 145.5$ $(\mathrm{C}(4) \operatorname{ArC}(1)), 165.0(C(2)=\mathrm{O}) ; \mathrm{m} / z$ (NSI) $404\left([\mathrm{M}+\mathrm{H}]^{+}, 70 \%\right) ; \mathrm{HRMS}(\mathrm{NSI}) \mathrm{C}_{24} \mathrm{H}_{22} \mathrm{NO}_{3} \mathrm{~S}^{+}\left([\mathrm{M}+\mathrm{H}]^{+}\right)$ requires 404.1314; found $404.1313(-0.2 \mathrm{ppm})$.

(3S,4R)-4-(4-bromophenyl)-1-[(4-methylbenzene)sulfonyl]-3-[(1E)-prop-1-en-1-yl]azetidin-2-one 26

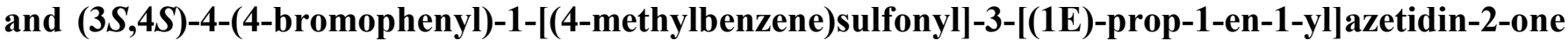
27

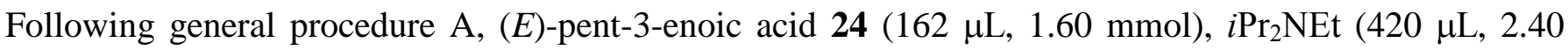
mmol) and pivaloyl chloride $(296 \mu \mathrm{L}, 2.40 \mathrm{mmol})$ in $\mathrm{CH}_{2} \mathrm{Cl}_{2}(10 \mathrm{~mL}), \mathrm{HBTM}-2.1(2 S, 3 R)-23$ (24.6 mg, $0.08 \mathrm{mmol}, 10 \mathrm{~mol} \%)$, imine $25(270 \mathrm{mg}, 0.80 \mathrm{mmol})$ and $i \operatorname{Pr}_{2} \mathrm{NEt}(348 \mu \mathrm{L}, 2.00 \mathrm{mmol})$ for $1.5 \mathrm{~h}$ at $\mathrm{rt}$ gave crude lactams $(3 S, 4 R)-26$ and $(3 S, 4 S)-27$ (68:32 dr). Chromatographic purification (eluent $\mathrm{Et}_{2} \mathrm{O}$ :petrol 20:80) gave lactam $(3 \mathrm{~S}, 4 R)-26(>98: 2 \mathrm{dr})$ as a colourless oil $(177 \mathrm{mg}, 53 \%)$ and lactam $(3 S, 4 S)-27(>98: 2 \mathrm{dr})$ as a white solid (91 mg, 27\%):

Data for lactam $(3 S, 4 R)$-26: Chiral HPLC Chiralpak AD-H (10\% IPA:hexane, flow rate $1 \mathrm{~mL} \mathrm{~min}^{-1}, 220$ $\left.\mathrm{nm}, 30^{\circ} \mathrm{C}\right) \mathrm{t}_{\mathrm{R}}(3 S, 4 R): 18.6 \mathrm{~min}, \mathrm{t}_{\mathrm{R}}(3 R, 4 S): 47.0 \mathrm{~min}, 79 \%$ ee; $v_{\max }(\mathrm{ATR}) / \mathrm{cm}^{-1} 3032,2965(\mathrm{C}-\mathrm{H}), 1794$ $(\mathrm{C}=\mathrm{O}), 1595,1366(\mathrm{~S}=\mathrm{O}), 1169(\mathrm{~S}=\mathrm{O}) ; \delta_{\mathrm{H}}\left(400 \mathrm{MHz}, \mathrm{CDCl}_{3}\right) 1.68(3 \mathrm{H}, \mathrm{ddd}, J$ 6.5, 1.6, $0.8 \mathrm{~Hz}$, $\left.\mathrm{CH}_{3} \mathrm{CH}=\mathrm{CH}\right), 2.44\left(3 \mathrm{H}, \mathrm{s}, \mathrm{ArCH}_{3}\right), 3.64(1 \mathrm{H}, \mathrm{ddt}, J$ 8.0, 3.3, $0.9 \mathrm{~Hz}, \mathrm{C}(3) H), 4.70(1 \mathrm{H}, \mathrm{d}, J 3.3 \mathrm{~Hz}$, $\mathrm{C}(4) H)$, 5.38-5.45 (1H, m, $\left.\mathrm{CH}_{3} \mathrm{CH}=\mathrm{CH}\right)$, 5.62-5.69 (1H, m, $\left.\mathrm{CH}_{3} \mathrm{CH}=\mathrm{CH}\right)$, 7.07-7.11 (2H, m, ArH), 7.26$7.29\left(2 \mathrm{H}, \mathrm{m}, \mathrm{SO}_{2} \operatorname{Ar}(3,5) H\right), 7.40-7.43(2 \mathrm{H}, \mathrm{m}, \mathrm{Ar} H), 7.64-7.67\left(2 \mathrm{H}, \mathrm{m}, \mathrm{SO}_{2} \mathrm{Ar}(2,6) H\right) ; \delta_{\mathrm{C}}(125 \mathrm{MHz}$, $\left.\mathrm{CDCl}_{3}\right) 18.2\left(\mathrm{CH}_{3} \mathrm{CH}=\mathrm{CH}\right), 21.8\left(\mathrm{ArCH}_{3}\right), 62.8(C(3)), 63.4 \quad(C(4)), 121.0 \quad\left(\mathrm{CH}_{3} \mathrm{CH}=\mathrm{CH}\right), 123.0$ $\left.(\mathrm{C}(4) \operatorname{ArC}(4)), 127.6(\operatorname{ArC}), 128.2(A r C), 130.0(\operatorname{ArC}), 132.1(\operatorname{ArC}), 133.1\left(\mathrm{CH}_{3} C \mathrm{H}=\mathrm{CH}\right)\right), 135.1$ (4ry $A r C), 135.5$ (4ry $A r C), 145.5(\mathrm{C}(4) \operatorname{ArC}(1)), 165.4(\mathrm{C}(2)=\mathrm{O}) ; m / z(\mathrm{APCI}) 420\left([\mathrm{M}+\mathrm{H}]^{+}, 98 \%\right)$; HRMS (APCI) $\mathrm{C}_{19} \mathrm{H}_{18} \mathrm{BrNO}_{3} \mathrm{~S}^{+}\left([\mathrm{M}+\mathrm{H}]^{+}\right)$requires 420.0264; found 420.0266 (+0.6 ppm).

Data for lactam $(3 S, 4 S)-27: \mathrm{mp} 92-94{ }^{\circ} \mathrm{C}$; Chiral HPLC Chiralpak AD-H (10\% IPA:hexane, flow rate 1 $\left.\mathrm{mL} \min ^{-1}, 211 \mathrm{~nm}, 30^{\circ} \mathrm{C}\right) \mathrm{t}_{\mathrm{R}}(3 S, 4 S): 20.9 \mathrm{~min}, \mathrm{t}_{\mathrm{R}}(3 R, 4 R): 23.0 \mathrm{~min}, 72 \%$ ee; $v_{\max }(\mathrm{ATR}) / \mathrm{cm}^{-1} 2941,2926$, 
$(\mathrm{C}-\mathrm{H}), 1786(\mathrm{C}=\mathrm{O}), 1487,1368(\mathrm{~S}=\mathrm{O}), 1125(\mathrm{~S}=\mathrm{O}) ; \delta_{\mathrm{H}}\left(400 \mathrm{MHz}, \mathrm{CDCl}_{3}\right) 1.51(3 \mathrm{H}, \mathrm{ddd}, J$ 6.6, $1.7,1.0$ $\left.\mathrm{Hz}, \mathrm{CH}_{3} \mathrm{CH}=\mathrm{CH}\right), 2.46\left(3 \mathrm{H}, \mathrm{s}, \mathrm{ArCH}_{3}\right), 4.16(1 \mathrm{H}, \mathrm{ddt}, J$ 7.8, 6.7, 1.1, C(3)H), $4.78(1 \mathrm{H}, \mathrm{ddq}, J$ 15.3, 7.7, $\left.1.7 \mathrm{~Hz}, \mathrm{CH}_{3} \mathrm{CH}=\mathrm{CH}\right), 5.17(1 \mathrm{H}, \mathrm{d}, J 6.7 \mathrm{~Hz}, \mathrm{C}(4) H), 5.69\left(1 \mathrm{H}\right.$, dqd, $\left.J 15.3,6.6,1.3 \mathrm{~Hz}, \mathrm{CH}_{3} \mathrm{CH}=\mathrm{CH}\right)$, 6.97-7.00 (2H, m, ArH), 7.31-7.33 (2H, m, SO $2 \operatorname{Ar}(3,5) H), 7.40-7.42(2 \mathrm{H}, \mathrm{m}, \operatorname{Ar} H), 7.75-7.77(2 \mathrm{H}, \mathrm{m}$, $\left.\mathrm{SO}_{2} \mathrm{Ar}(2,6) H\right) ; \delta_{\mathrm{C}}\left(125 \mathrm{MHz}, \mathrm{CDCl}_{3}\right) 18.2\left(\mathrm{CH}_{3} \mathrm{CH}=\mathrm{CH}\right), 21.9\left(\mathrm{ArCH} \mathrm{H}_{3}\right), 58.3(C(3)), 61.1(C(4)), 119.4$ $\left(\mathrm{CH}_{3} \mathrm{CH}=\mathrm{CH}\right), 122.7(\mathrm{C}(4) \operatorname{ArC}(4)), 127.7(\operatorname{ArC}), 129.1(\operatorname{ArC}), 130.1(\operatorname{ArC}), 131.7(\operatorname{ArC}), 133.2(4 \mathrm{ry} A r C)$, $133.9\left(\mathrm{CH}_{3} C \mathrm{H}=\mathrm{CH}\right)$ ), 135.6 (4ry $\left.A r C\right), 145.7(\mathrm{C}(4) \operatorname{ArC}(1)), 165.4(C(2)=\mathrm{O}) ; m / z(\mathrm{NSI}) 420\left([\mathrm{M}+\mathrm{H}]^{+}\right.$, 100\%); HRMS (NSI) $\mathrm{C}_{19} \mathrm{H}_{18} \mathrm{BrNO}_{3} \mathrm{~S}^{+}\left([\mathrm{M}+\mathrm{H}]^{+}\right)$requires 420.0264; found 420.0263 (-0.1 ppm).

Reaction carried out for $1.5 \mathrm{~h}$ at $-78{ }^{\circ} \mathrm{C}$ gave crude lactams (3S,4R)-26:(3S,4S)-27 (71:29 dr). Chromatographic purification (eluent $\mathrm{Et}_{2} \mathrm{O}$ :petrol 20:80) gave lactam $(3 S, 4 R)-26(>98: 2 \mathrm{dr})$ as a colourless oil (179 mg, 53\%) with identical spectroscopic properties as before in $97 \%$ ee; $[\alpha]_{D}^{20} 0.6\left(c 0.5, \mathrm{CH}_{2} \mathrm{Cl}_{2}\right)$ and lactam $(3 S, 4 S)-27(>98: 2 \mathrm{dr})$ as a white solid $(35.6 \mathrm{mg}, 11 \%)$ with identical spectroscopic properties as before in $>99 \%$ ee; $[\alpha]_{D}^{20}-14.6\left(c 0.5, \mathrm{CH}_{2} \mathrm{Cl}_{2}\right)$.

\section{(3S,4R)-4-(4-methoxyphenyl)-1-[(4-methylbenzene)sulfonyl]-3-[(1E)-prop-1-en-1-yl]azetidin-2-one}

\section{8}

Following general procedure A, (E)-pent-3-enoic acid $24(162 \mu \mathrm{L}, 1.60 \mathrm{mmol}), i \mathrm{Pr}_{2} \mathrm{NEt}(420 \mu \mathrm{L}, 2.40$ mmol) and pivaloyl chloride $(296 \mu \mathrm{L}, 2.40 \mathrm{mmol})$ in $\mathrm{CH}_{2} \mathrm{Cl}_{2}(10 \mathrm{~mL})$, HBTM-2.1 (2S,3R)-23 (24.6 mg, $0.08 \mathrm{mmol}, 10 \mathrm{~mol} \%)$, imine $53(231 \mathrm{mg}, 0.80 \mathrm{mmol})$ and $i \operatorname{Pr}_{2} \mathrm{NEt}(348 \mu \mathrm{L}, 2.00 \mathrm{mmol})$ for $1.5 \mathrm{~h}$ at $\mathrm{rt}$ gave crude lactam $(3 S, 4 R)-\mathbf{2 8}(80: 20 \mathrm{dr})$. Chromatographic purification (eluent $\mathrm{Et}_{2} \mathrm{O}$ :petrol 30:70) gave lactam $(3 S, 4 R)-28(96: 4 \mathrm{dr})$ as a yellow oil $(220 \mathrm{mg}, 74 \%) ;[\alpha]_{D}^{20}-11.2\left(c 0.5, \mathrm{CH}_{2} \mathrm{Cl}_{2}\right) ;$ Chiral HPLC Chiralpak AD-H (10\% IPA:hexane, flow rate $\left.1 \mathrm{~mL} \mathrm{~min}^{-1}, 211 \mathrm{~nm}, 30{ }^{\circ} \mathrm{C}\right) \mathrm{t}_{\mathrm{R}}(3 S, 4 R): 17.6 \mathrm{~min}, \mathrm{t}_{\mathrm{R}}(3 R, 4 S)$ : $53.0 \mathrm{~min}, 86 \%$ ee; $v_{\max }(\mathrm{ATR}) / \mathrm{cm}^{-1} 2966(\mathrm{C}-\mathrm{H}), 1790(\mathrm{C}=\mathrm{O}), 1612,1516,1364(\mathrm{~S}=\mathrm{O}), 1167(\mathrm{~S}=\mathrm{O})$; Data for major diastereomer: $\delta_{\mathrm{H}}\left(500 \mathrm{MHz}, \mathrm{CDCl}_{3}\right) 1.68\left(3 \mathrm{H}, \mathrm{ddd}, J 6.5,1.6,0.8 \mathrm{~Hz}, \mathrm{CH}_{3} \mathrm{CH}=\mathrm{CH}\right), 2.42(3 \mathrm{H}, \mathrm{s}$, $\left.\mathrm{ArCH}_{3}\right), 3.67(1 \mathrm{H}, \mathrm{ddt}, J$ 8.0, 3.3, $0.9 \mathrm{~Hz}, \mathrm{C}(3) H), 3.80\left(3 \mathrm{H}, \mathrm{s}, \mathrm{OCH}_{3}\right), 4.73(1 \mathrm{H}, \mathrm{d}, J 3.3 \mathrm{~Hz}, \mathrm{C}(4) H), 5.43$ 
$\left(1 \mathrm{H}, \mathrm{ddq}, J 15.3,8.0,1.6 \mathrm{~Hz}, \mathrm{CH}_{3} \mathrm{CH}=\mathrm{CH}\right), 5.63-5.70\left(1 \mathrm{H}, \mathrm{m}, \mathrm{CH}_{3} \mathrm{CH}=\mathrm{CH}\right), 6.80-6.81(2 \mathrm{H}, \mathrm{m}$, $\mathrm{C}(4) \operatorname{Ar}(3,5) H), 7.12-7.14(2 \mathrm{H}, \mathrm{m}, \mathrm{C}(4) \operatorname{Ar}(2,6) H), 7.24\left(2 \mathrm{H}, \mathrm{d}, J 8.6 \mathrm{~Hz}, \mathrm{SO}_{2} \operatorname{Ar}(3,5) H\right), 7.60-7.61(2 \mathrm{H}, \mathrm{m}$, $\left.\mathrm{SO}_{2} \mathrm{Ar}(2,6) H\right) ; \delta_{\mathrm{C}}\left(125 \mathrm{MHz}, \mathrm{CDCl}_{3}\right) 18.2\left(\mathrm{CH}_{3} \mathrm{CH}=\mathrm{CH}\right), 21.8\left(\mathrm{ArCH}_{3}\right), 55.5\left(\mathrm{OCH}_{3}\right), 62.6(C(3)), 64.0$ $(C(4)), 114.3(\mathrm{C}(4) \operatorname{ArC}(3,5)), 121.4\left(\mathrm{CH}_{3} \mathrm{CH}=C \mathrm{H}\right), 127.6\left(\mathrm{SO}_{2} \operatorname{ArC}(2,6)\right), 127.8(\mathrm{C}(4) \operatorname{ArC}(1)), 128.2$ (C(4) $\left.\operatorname{ArC}(2,6)), 129.8\left(\mathrm{SO}_{2} \operatorname{ArC}(3,5)\right), 132.6\left(\mathrm{CH}_{3} C \mathrm{H}=\mathrm{CH}\right)\right), 135.9\left(\mathrm{SO}_{2} \operatorname{ArC}(1)\right), 145.1 \quad\left(\mathrm{SO}_{2} \operatorname{ArC}(4)\right)$, $160.2(\mathrm{C}(4) \operatorname{ArC}(4)), 165.9(C(2)=\mathrm{O}) ; \mathrm{m} / z(\mathrm{ESI}) 394\left([\mathrm{M}+\mathrm{Na}]^{+}, 80 \%\right) ; \mathrm{HRMS}(\mathrm{ESI}) \mathrm{C}_{20} \mathrm{H}_{21} \mathrm{NNaO}_{4} \mathrm{~S}^{+}$ $\left([\mathrm{M}+\mathrm{Na}]^{+}\right)$requires 394.1089; found $394.1075(-2.2 \mathrm{ppm})$.

\section{(3S,4R)-1-[(4-methylbenzene)sulfonyl]-3-[(1E)-prop-1-en-1-yl]-4-[4-}

(trifluoromethyl)phenyl]azetidin-2-one 29

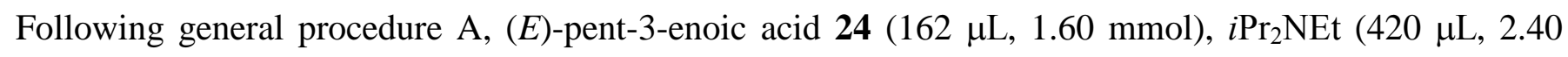
mmol) and pivaloyl chloride $(296 \mu \mathrm{L}, 2.40 \mathrm{mmol})$ in $\mathrm{CH}_{2} \mathrm{Cl}_{2}(10 \mathrm{~mL})$, HBTM-2.1 (2S,3R)-23 (24.6 mg, $0.08 \mathrm{mmol}, 10 \mathrm{~mol} \%)$, imine $54(262 \mathrm{mg}, 0.80 \mathrm{mmol})$ and $i \operatorname{Pr}_{2} \mathrm{NEt}(348 \mu \mathrm{L}, 2.00 \mathrm{mmol})$ for $1.5 \mathrm{~h}$ at $\mathrm{rt}$ gave crude lactam $(3 S, 4 R)$-29 (87:13 dr). Chromatographic purification (eluent Et $_{2} \mathrm{O}$ :petrol 20:80) gave lactam $(3 S, 4 R)-29(>98: 2 \mathrm{dr})$ as a colourless oil $(151 \mathrm{mg}, 46 \%) ;[\alpha]_{D}^{20}+1.0\left(c 0.5, \mathrm{CH}_{2} \mathrm{Cl}_{2}\right)$; Chiral HPLC Chiralpak AD-H (10\% IPA:hexane, flow rate $\left.1 \mathrm{~mL} \mathrm{~min}^{-1}, 211 \mathrm{~nm}, 30^{\circ} \mathrm{C}\right) \mathrm{t}_{\mathrm{R}}(3 S, 4 R): 20.3 \mathrm{~min}, \mathrm{t}_{\mathrm{R}}(3 R, 4 S)$ : $48.4 \mathrm{~min}, 44 \% e e ; v_{\max }(\mathrm{ATR}) / \mathrm{cm}^{-1} 2970(\mathrm{C}-\mathrm{H}), 1796(\mathrm{C}=\mathrm{O}), 1597,1323(\mathrm{~S}=\mathrm{O}), 1165(\mathrm{~S}=\mathrm{O})$; Data for major diastereomer: $\delta_{\mathrm{H}}\left(400 \mathrm{MHz}, \mathrm{CDCl}_{3}\right) 1.69\left(3 \mathrm{H}\right.$, ddd, $\left.J 6.5,1.6,0.8 \mathrm{~Hz}, \mathrm{CH}_{3} \mathrm{CH}=\mathrm{CH}\right), 2.44(3 \mathrm{H}, \mathrm{s}$, $\left.\mathrm{ArCH}_{3}\right), 3.67(1 \mathrm{H}, \mathrm{ddt}, J$ 8.0, 3.3, $0.9 \mathrm{~Hz}, \mathrm{C}(3) H), 4.79(1 \mathrm{H}, \mathrm{d}, J 3.3 \mathrm{~Hz}, \mathrm{C}(4) H), 5.39-5.46(1 \mathrm{H}, \mathrm{m}$, $\left.\mathrm{CH}_{3} \mathrm{CH}=\mathrm{CH}\right), 5.64-5.73\left(1 \mathrm{H}, \mathrm{m}, \mathrm{CH}_{3} \mathrm{CH}=\mathrm{CH}\right), 7.25-7.28\left(2 \mathrm{H}, \mathrm{m}, \mathrm{SO}_{2} \mathrm{Ar}(3,5) H\right), 7.35(2 \mathrm{H}, \mathrm{d}, J 8.3 \mathrm{~Hz}$, $\mathrm{C}(4) \operatorname{Ar}(3,5) H), 7.55(2 \mathrm{H}, \mathrm{d}, J 8.1 \mathrm{~Hz}, \mathrm{C}(4) \operatorname{Ar}(2,6) H), 7.65-7.68\left(2 \mathrm{H}, \mathrm{m}, \mathrm{SO}_{2} \mathrm{Ar}(2,6) H\right) ; \delta_{\mathrm{C}}(125 \mathrm{MHz}$, $\left.\mathrm{CDCl}_{3}\right) 18.2\left(\mathrm{CH}_{3} \mathrm{CH}=\mathrm{CH}\right), 21.8\left(\mathrm{ArCH}_{3}\right), 63.0(C(3)$ or $C(4)), 63.2(C(3)$ or $C(4)), 120.8\left(\mathrm{CH}_{3} \mathrm{CH}=\mathrm{CH}\right)$, 123.9 (q, J $\left.271 \mathrm{~Hz}, C \mathrm{~F}_{3}\right), 125.9$ (q, J $\left.3.5 \mathrm{~Hz}, \mathrm{C}(4) \operatorname{ArC}(3,5)\right), 126.9(\mathrm{C}(4) \operatorname{ArC}(2,6)), 127.6\left(\mathrm{SO}_{2} \operatorname{ArC}(2,6)\right)$, $130.0\left(\mathrm{SO}_{2} \operatorname{ArC}(3,5)\right), 131.2(\mathrm{q}, J 32.5 \mathrm{~Hz}, \mathrm{C}(4) \operatorname{ArC}(4)), 133.4\left(\mathrm{CH}_{3} C \mathrm{H}=\mathrm{CH}\right), 135.4\left(\mathrm{SO}_{2} \operatorname{ArC}(1)\right), 140.2$ $(\mathrm{C}(4) \operatorname{ArC}(1)), 145.7\left(\mathrm{SO}_{2} \operatorname{ArC}(4)\right), 165.2(C(2)=\mathrm{O}) ; \delta_{\mathrm{F}}\left(376 \mathrm{MHz}, \mathrm{CDCl}_{3}\right)-63.3(\mathrm{CF}) ; m / z(\mathrm{NSI}) 410$ $\left([\mathrm{M}+\mathrm{H}]^{+}, 15 \%\right)$; HRMS (NSI) $\mathrm{C}_{20} \mathrm{H}_{19} \mathrm{~F}_{3} \mathrm{NO}_{3} \mathrm{~S}^{+}\left([\mathrm{M}+\mathrm{H}]^{+}\right)$requires 410.1032 ; found 410.1030 (-0.5 ppm) 


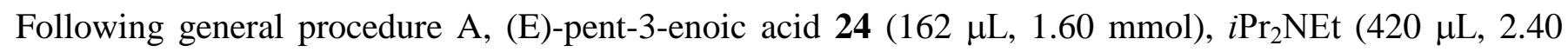
mmol) and pivaloyl chloride $(296 \mu \mathrm{L}, 2.40 \mathrm{mmol})$ in $\mathrm{CH}_{2} \mathrm{Cl}_{2}(10 \mathrm{~mL})$, HBTM-2.1 (2S,3R)-23 (24.6 mg, $0.08 \mathrm{mmol}, 10 \mathrm{~mol} \%)$, imine 55 (199 mg, $0.80 \mathrm{mmol})$ and $i \operatorname{Pr}_{2} \mathrm{NEt}(348 \mu \mathrm{L}, 2.00 \mathrm{mmol})$ for $1.5 \mathrm{~h}$ at $\mathrm{rt}$ gave crude lactam $(3 S, 4 R)-30$ (73:27 dr). Chromatographic purification (eluent Et ${ }_{2} \mathrm{O}$ :petrol 20:80) gave lactam $(3 S, 4 R)-30(95: 5 \mathrm{dr})$ as a white solid (171 mg, 65\%); mp 137-139 ${ }^{\circ} \mathrm{C}$; Chiral HPLC Chiralpak AD-

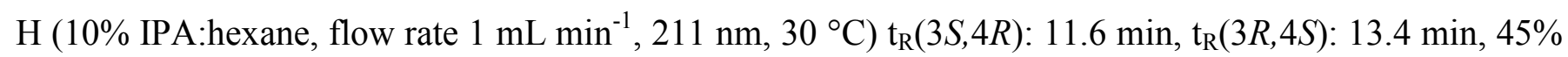
ee; $v_{\max }(\mathrm{ATR}) / \mathrm{cm}^{-1} 2976(\mathrm{C}-\mathrm{H}), 1788(\mathrm{C}=\mathrm{O}), 1595,1362(\mathrm{~S}=\mathrm{O}), 1165(\mathrm{~S}=\mathrm{O})$; Data for major diastereoisomer: $\delta_{\mathrm{H}}\left(400 \mathrm{MHz}, \mathrm{CDCl}_{3}\right) 1.71\left(3 \mathrm{H}, \mathrm{ddd}, J 6.5,1.6,0.9 \mathrm{~Hz}, \mathrm{CH}_{3} \mathrm{CH}=\mathrm{CH}\right), 2.42(3 \mathrm{H}, \mathrm{s}$, $\left.\mathrm{ArCH}_{3}\right), 4.00-4.03(1 \mathrm{H}, \mathrm{m}, \mathrm{C}(3) H), 4.86-4.87(1 \mathrm{H}, \mathrm{m}, \mathrm{C}(4) H), 5.45-5.52\left(1 \mathrm{H}, \mathrm{m}, \mathrm{CH}_{3} \mathrm{CH}=\mathrm{CH}\right), 5.71-5.80$ $\left(1 \mathrm{H}, \mathrm{m}, \mathrm{CH}_{3} \mathrm{CH}=\mathrm{CH}\right), 6.35(1 \mathrm{H}, \mathrm{dd}, J 3.3,1.9 \mathrm{~Hz}, \mathrm{C}(4) \mathrm{ArC}(4) H), 6.50(1 \mathrm{H}, \mathrm{dd}, J 3.3,0.7 \mathrm{~Hz}$, $\mathrm{C}(4) \operatorname{ArC}(3) H), 7.20 \quad(1 \mathrm{H}, \mathrm{dt}, J 1.0,0.5 \mathrm{~Hz}, \mathrm{C}(4) \operatorname{ArC}(5) H), 7.22-7.24\left(2 \mathrm{H}, \mathrm{m}, \mathrm{SO}_{2} \mathrm{Ar}(3,5) H\right), 7.52-7.55$ $\left(2 \mathrm{H}, \mathrm{m}, \mathrm{SO}_{2} \mathrm{Ar}(2,6) H\right) ; \delta_{\mathrm{C}}\left(125 \mathrm{MHz}, \mathrm{CDCl}_{3}\right) 18.2\left(\mathrm{CH}_{3} \mathrm{CH}=\mathrm{CH}\right), 21.8\left(\mathrm{ArCH}_{3}\right), 56.7(C(3)), 58.7(C(4))$, $110.9(\mathrm{C}(4) A r C), 112.0(\mathrm{C}(4) A r C), 121.1\left(\mathrm{CH}_{3} \mathrm{CH}=C \mathrm{H}\right), 127.4\left(\mathrm{SO}_{2} \operatorname{ArC}(2,6)\right), 129.8\left(\mathrm{SO}_{2} \operatorname{ArC}(3,5)\right)$, $\left.133.0\left(\mathrm{CH}_{3} C \mathrm{H}=\mathrm{CH}\right)\right), 135.7(\mathrm{C}(4) \operatorname{ArC}(1)), 143.5(\mathrm{C}(4) \operatorname{ArC}(5)), 145.0\left(\mathrm{SO}_{2} \operatorname{ArC}(1)\right), 147.6\left(\mathrm{SO}_{2} \operatorname{ArC}(4)\right)$, $164.9(C(2)=\mathrm{O}) ; m / z(\mathrm{APCI}) 332\left([\mathrm{M}+\mathrm{H}]^{+}, 100 \%\right)$; HRMS (APCI) $\mathrm{C}_{17} \mathrm{H}_{18} \mathrm{NO}_{4} \mathrm{~S}^{+}\left([\mathrm{M}+\mathrm{H}]^{+}\right)$requires 332.0951; found $332.0954(+0.9 \mathrm{ppm})$.

Reaction carried out for $1.5 \mathrm{~h}$ at $-78{ }^{\circ} \mathrm{C}$ gave crude lactam $(3 S, 4 R)-30$ (63:37 dr). Chromatographic purification (eluent $\mathrm{Et}_{2} \mathrm{O}$ :petrol 20:80) gave lactam $(3 S, 4 R)-30(95: 5 \mathrm{dr})$ as a white solid (126 $\left.\mathrm{mg}, 48 \%\right)$ with identical spectroscopic properties as before in 95\% ee; $[\alpha]_{D}^{20}-6.4\left(c 0.5, \mathrm{CH}_{2} \mathrm{Cl}_{2}\right)$.

(3S,4R)-1-[(4-methylbenzene)sulfonyl]-4-(naphthalen-2-yl)-3-[(1E)-prop-1-en-1-yl]azetidin-2-one 31 and $\quad(3 S, 4 S)-1-[(4-m e t h y l b e n z e n e) s u l f o n y l]-4-(n a p h t h a l e n-2-y l)-3-[(1 E)-p r o p-1-e n-1-y l]$ azetidin-2one 75 


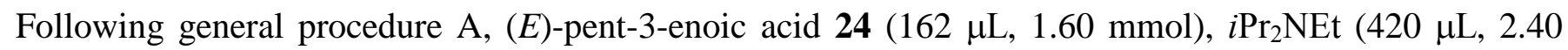
mmol) and pivaloyl chloride $(296 \mu \mathrm{L}, 2.40 \mathrm{mmol})$ in $\mathrm{CH}_{2} \mathrm{Cl}_{2}(10 \mathrm{~mL}), \mathrm{HBTM}-2.1(2 S, 3 R)-23$ (24.6 mg, $0.08 \mathrm{mmol}, 10 \mathrm{~mol} \%)$, imine $56(247 \mathrm{mg}, 0.80 \mathrm{mmol})$ and $i \operatorname{Pr}_{2} \mathrm{NEt}(348 \mu \mathrm{L}, 2.00 \mathrm{mmol})$ for $1.5 \mathrm{~h}$ at $\mathrm{rt}$ gave crude lactams $(3 S, 4 R)-31$ and $(3 S, 4 S)-75(67: 33 \mathrm{dr})$. Chromatographic purification (eluent $\mathrm{Et}_{2} \mathrm{O}$ :petrol 20:80) gave lactam $(3 S, 4 R)-31(>98: 2 \mathrm{dr})$ as a colourless oil $(188 \mathrm{mg}, 60 \%)$ and lactam (3S,4S)-75 (94:6 dr) as a colourless oil (96 mg, 31\%):

Data for lactam $(3 S, 4 R)-31$ : Chiral HPLC Chiralpak AD-H (10\% IPA:hexane, flow rate $1 \mathrm{~mL} \mathrm{~min}^{-1}, 211$ $\left.\mathrm{nm}, 30{ }^{\circ} \mathrm{C}\right) \mathrm{t}_{\mathrm{R}}(3 S, 4 R): 17.1 \mathrm{~min}, \mathrm{t}_{\mathrm{R}}(3 R, 4 S): 37.0 \mathrm{~min}, 81 \% e e ; v_{\max }(\mathrm{ATR}) / \mathrm{cm}^{-1} 2972(\mathrm{C}-\mathrm{H}), 1792(\mathrm{C}=\mathrm{O})$, 1699, $1364(\mathrm{~S}=\mathrm{O}), 1167(\mathrm{~S}=\mathrm{O}) ; \delta_{\mathrm{H}}\left(500 \mathrm{MHz}, \mathrm{CDCl}_{3}\right) 1.71\left(3 \mathrm{H}, \mathrm{ddd}, J 6.5,1.5,0.7 \mathrm{~Hz}, \mathrm{CH} \mathrm{CH}_{3} \mathrm{CH}\right)$, $2.38\left(3 \mathrm{H}, \mathrm{s}, \mathrm{ArCH}_{3}\right), 3.77(1 \mathrm{H}, \mathrm{ddd}, J 7.9,2.4,0.8 \mathrm{~Hz}, \mathrm{C}(3) H), 4.95(1 \mathrm{H}, \mathrm{d}, J 3.3 \mathrm{~Hz}, \mathrm{C}(4) H), 5.48-5.53$ $\left(1 \mathrm{H}, \mathrm{m}, \mathrm{CH}_{3} \mathrm{CH}=\mathrm{CH}\right)$, 5.68-5.72 $\left(1 \mathrm{H}, \mathrm{m}, \mathrm{CH}_{3} \mathrm{CH}=\mathrm{CH}\right), 7.14-7.16\left(2 \mathrm{H}, \mathrm{m}, \mathrm{SO}_{2} \mathrm{Ar}(3,5) H\right), 7.23(1 \mathrm{H}, \mathrm{dd}, J$ 8.5, $1.8 \mathrm{~Hz}, \mathrm{C}(4) \mathrm{Ar} H), 7.49-7.53(2 \mathrm{H}, \mathrm{m}, \mathrm{Ar} H), 7.60-7.63\left(2 \mathrm{H}, \mathrm{m}, \mathrm{SO}_{2} \mathrm{Ar}(2,6) H\right), 7.70-7.72(1 \mathrm{H}, \mathrm{m}$, $\mathrm{C}(4) \operatorname{Ar}(1) H), 7.74\left(1 \mathrm{H}, \mathrm{d}, J\right.$ 8.5, C(4)ArH), 7.81-7.84 (1H, m, C(4)ArH); $\delta_{\mathrm{C}}\left(125 \mathrm{MHz}, \mathrm{CDCl}_{3}\right) 18.2$ $\left(\mathrm{CH}_{3} \mathrm{CH}=\mathrm{CH}\right), 21.7\left(\mathrm{ArCH}_{3}\right), 62.9(C(3)), 64.4(C(4)), 121.3\left(\mathrm{CH}_{3} \mathrm{CH}=C \mathrm{H}\right), 123.3(\operatorname{ArC}), 126.6(\operatorname{ArC})$, $126.7(\mathrm{ArC}), 126.8(\mathrm{ArC}), 127.6\left(\mathrm{SO}_{2} \operatorname{ArC}(2,6)\right), 127.8(\operatorname{ArC}), 128.1(\operatorname{ArC}), 129.0(\operatorname{ArC}), 129.8$ $\left.\left(\mathrm{SO}_{2} \operatorname{ArC}(3.5)\right), 132.9\left(\mathrm{CH}_{3} \mathrm{CH}=\mathrm{CH}\right)\right), 133.0$ (4ry $\left.A r C\right), 133.1$ (4ry $\left.A r C\right), 133.5$ (4ry $\left.A r C\right), 135.7$ (4ry $\left.A r C\right)$, $145.4(\mathrm{C}(4) \operatorname{ArC}(1)), 165.7(C(2)=\mathrm{O}) ; \mathrm{m} / z$ (APCI) $392\left([\mathrm{M}+\mathrm{H}]^{+}, 26 \%\right) ; \mathrm{HRMS}\left(\right.$ APCI) $\mathrm{C}_{23} \mathrm{H}_{22} \mathrm{NO}_{3} \mathrm{~S}^{+}$ $\left([\mathrm{M}+\mathrm{H}]^{+}\right)$requires 392.1315 ; found $392.1318(+0.8 \mathrm{ppm})$.

Data for lactam $(3 S, 4 S)$-75: Chiral HPLC Chiralcel OD-H (10\% IPA:hexane, flow rate $1 \mathrm{~mL} \mathrm{~min}^{-1}, 211$ $\left.\mathrm{nm}, 30{ }^{\circ} \mathrm{C}\right) \mathrm{t}_{\mathrm{R}}(3 S, 4 S): 19.2 \mathrm{~min}, \mathrm{t}_{\mathrm{R}}(3 R, 4 R): 25.0 \mathrm{~min}, 81 \% e e ; v_{\max }(\mathrm{ATR}) / \mathrm{cm}^{-1} 2972(\mathrm{C}-\mathrm{H}), 1790(\mathrm{C}=\mathrm{O})$, 1597, $1364(\mathrm{~S}=\mathrm{O}), 1169(\mathrm{~S}=\mathrm{O}) ; \delta_{\mathrm{H}}\left(400 \mathrm{MHz}, \mathrm{CDCl}_{3}\right) 1.43\left(3 \mathrm{H}, \mathrm{ddd}, J\right.$ 6.6, 1.7, $\left.1.0 \mathrm{~Hz}, \mathrm{CH} \mathrm{CH}_{3} \mathrm{CH}\right)$, $2.43\left(3 \mathrm{H}, \mathrm{s}, \mathrm{ArCH}_{3}\right), 4.26(1 \mathrm{H}, \mathrm{ddq}, J$ 7.8, 6.8, 1.0, C(3)H), $4.84(1 \mathrm{H}, \mathrm{ddq}, J 15.3,7.8,1.7 \mathrm{~Hz}$, $\left.\mathrm{CH}_{3} \mathrm{CH}=\mathrm{CH}\right), 5.42(1 \mathrm{H}, \mathrm{d}, J 6.7 \mathrm{~Hz}, \mathrm{C}(4) H), 5.66-5.75\left(1 \mathrm{H}, \mathrm{m}, \mathrm{CH}_{3} \mathrm{CH}=\mathrm{CH}\right), 7.13(1 \mathrm{H}, \mathrm{dd}, J 8.5,1.9 \mathrm{~Hz}$, $\mathrm{C}(4) \mathrm{Ar} H), 7.24-7.27\left(2 \mathrm{H}, \mathrm{m}, \mathrm{SO}_{2} \mathrm{Ar}(3,5) H\right), 7.48-7.52(2 \mathrm{H}, \mathrm{m}, \mathrm{Ar} H), 7.55(1 \mathrm{H}, \mathrm{dd}, J$ 1.2, 0.5, $\mathrm{C}(4) \operatorname{Ar}(1) H), 7.66-7.68(1 \mathrm{H}, \mathrm{m}, \mathrm{C}(4) \operatorname{Ar} H), 7.72-7.77(3 \mathrm{H}, \mathrm{m}, \operatorname{Ar} H), 7.81-7.84(1 \mathrm{H}, \mathrm{m}, \mathrm{C}(4) \operatorname{Ar} H) ; \delta_{\mathrm{C}}(100$ 
$\left.\mathrm{MHz}, \mathrm{CDCl}_{3}\right) 18.1\left(\mathrm{CH}_{3} \mathrm{CH}=\mathrm{CH}\right), 21.8\left(\mathrm{ArCH}_{3}\right), 58.6(C(3)), 62.0(C(4)), 119.7\left(\mathrm{CH}_{3} \mathrm{CH}=C \mathrm{H}\right), 124.8$ (ArC), $126.6(A r C), 126.6(\operatorname{ArC}), 127.1(\operatorname{ArC}), 127.8(\operatorname{ArC}), 127.8\left(\mathrm{SO}_{2} \operatorname{ArC}(2,6)\right), 128.1(\operatorname{ArC}), 128.3$ (ArC), $130.0\left(\mathrm{SO}_{2} \mathrm{ArC}(3,5)\right), 131.5$ (4ry $\left.\mathrm{ArC}\right), 133.0$ (4ry $\left.\mathrm{ArC}\right), 133.4$ (4ry $\left.\mathrm{ArC}\right), 133.6\left(\mathrm{CH}_{3} \mathrm{CH}=\mathrm{CH}\right)$, 135.9 (4ry $A r C), 145.4(\mathrm{C}(4) \operatorname{ArC}(1)), 165.7(C(2)=\mathrm{O}) ; m / z(\mathrm{APCI}) 392\left([\mathrm{M}+\mathrm{H}]^{+}, 83 \%\right)$; HRMS (APCI) $\mathrm{C}_{23} \mathrm{H}_{22} \mathrm{NO}_{3} \mathrm{~S}^{+}\left([\mathrm{M}+\mathrm{H}]^{+}\right)$requires 392.1315; found $392.1316(+0.3 \mathrm{ppm})$.

Reaction carried out for $1.5 \mathrm{~h}$ at $-78{ }^{\circ} \mathrm{C}$ gave crude lactams (3S,4R)-31:(3S,4S)-75 (76:24 dr). Chromatographic purification (eluent $\mathrm{Et}_{2} \mathrm{O}$ :petrol 20:80) gave lactam $(3 S, 4 R)-31$ (>98:2 $\mathrm{dr}$ ) as a colourless oil (201 mg, 64\%) with identical spectroscopic properties as before in 97\% ee; $[\alpha]_{D}^{20}-10.6\left(c 0.5, \mathrm{CH}_{2} \mathrm{Cl}_{2}\right)$ and lactam $(3 S, 4 S)-75(95: 5 \mathrm{dr})$ as a colourless oil $(69.0 \mathrm{mg}, 22 \%)$ with identical spectroscopic properties as before in $99 \% e e ;[\alpha]_{D}^{20}+0.6\left(c 0.5, \mathrm{CH}_{2} \mathrm{Cl}_{2}\right)$.

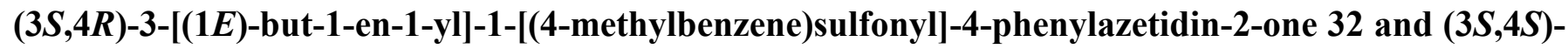

\section{3-[(1E)-but-1-en-1-yl]-1-[(4-methylbenzene)sulfonyl]-4-phenylazetidin-2-one 76}

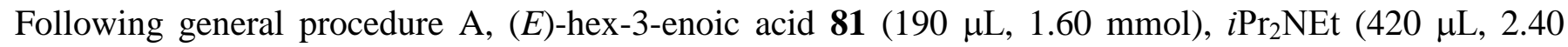
mmol) and pivaloyl chloride $(296 \mu \mathrm{L}, 2.40 \mathrm{mmol})$ in $\mathrm{CH}_{2} \mathrm{Cl}_{2}(10 \mathrm{~mL})$, HBTM-2.1 (2S,3R)-23 (24.6 mg, $0.08 \mathrm{mmol}, 10 \mathrm{~mol} \%$ ), imine 19 (207 mg, $0.80 \mathrm{mmol})$ and $i \operatorname{Pr}_{2} \mathrm{NEt}(348 \mu \mathrm{L}, 2.00 \mathrm{mmol})$ for $1.5 \mathrm{~h}$ at $\mathrm{rt}$ gave crude lactams $(3 S, 4 R)-32$ and $(3 S, 4 S)-76(84: 16 \mathrm{dr})$. Chromatographic purification (eluent $\mathrm{Et}_{2} \mathrm{O}$ :petrol 20:80) gave lactam $(3 S, 4 R)-32(>98: 2 \mathrm{dr})$ as a colourless oil (158 $\left.\mathrm{mg}, 55 \%\right)$ and lactam $(3 S, 4 S)-76(98: 2 \mathrm{dr})$ as a white solid $(22 \mathrm{mg}, 8 \%)$ :

Data for lactam $(3 S, 4 R)$-32: Chiral HPLC Chiralpak AD-H (10\% IPA:hexane, flow rate $1 \mathrm{~mL} \mathrm{~min}^{-1}, 211$ $\left.\mathrm{nm}, 30{ }^{\circ} \mathrm{C}\right) \mathrm{t}_{\mathrm{R}}(3 S, 4 R): 11.5 \mathrm{~min}, \mathrm{t}_{\mathrm{R}}(3 R, 4 S): 17.8 \mathrm{~min}, 81 \%$ ee; $v_{\max }(\mathrm{ATR}) / \mathrm{cm}^{-1} 2967(\mathrm{C}-\mathrm{H}), 1794(\mathrm{C}=\mathrm{O})$, 1699, $1366(\mathrm{~S}=\mathrm{O}), 1169(\mathrm{~S}=\mathrm{O})$; Data for major diastereomer: $\delta_{\mathrm{H}}\left(500 \mathrm{MHz}, \mathrm{CDCl}_{3}\right) 0.95(3 \mathrm{H}, \mathrm{t}, J 7.5 \mathrm{~Hz}$, $\left.\mathrm{CH}_{2} \mathrm{CH}_{3}\right), 2.00-2.07\left(2 \mathrm{H}, \mathrm{m}, \mathrm{CH}_{2} \mathrm{CH}_{3}\right), 2.42\left(3 \mathrm{H}, \mathrm{s}, \mathrm{ArCH}_{3}\right), 3.70(1 \mathrm{H}, \mathrm{ddt}, J$ 7.8, 2.4, $0.9 \mathrm{~Hz}, \mathrm{C}(3) H)$, $4.77(1 \mathrm{H}, \mathrm{d}, J 3.3 \mathrm{~Hz}, \mathrm{C}(4) H), 5.42(1 \mathrm{H}, \mathrm{ddt}, J 15.4,7.8,1.6 \mathrm{~Hz}, \mathrm{EtCH}=\mathrm{CH}), 5.70(1 \mathrm{H}, \mathrm{dtd}, J 15.4,6.3$, 1.1 Hz, EtCH=CH), 7.21-7.25 (4H, m, ArH), 7.27-7.34 (3H, m, ArH), 7.62-7.64 (2H, m, $\left.\mathrm{SO}_{2} \operatorname{Ar}(2,6) H\right)$; 
$\delta_{\mathrm{C}}\left(125 \mathrm{MHz}, \mathrm{CDCl}_{3}\right) 13.1\left(\mathrm{CH}_{2} \mathrm{CH}_{3}\right), 21.8\left(\mathrm{ArCH}_{3}\right), 25.6\left(\mathrm{CH}_{2} \mathrm{CH}_{3}\right), 62.6(C(3)$ or $C(4)), 64.2(C(3)$ or $C(4)), 119.1 \quad(\mathrm{EtCH}=C H), 126.7(\mathrm{C}(4) \operatorname{ArC}(2,6)), 127.6 \quad\left(\mathrm{SO}_{2} \operatorname{ArC}(2,6)\right), 128.9(\mathrm{C}(4) \operatorname{ArC}(3,5)), 129.0$ (C(4)ArC(4)), $129.9\left(\mathrm{SO}_{2} \operatorname{ArC}(3.5)\right), 135.7$ (4ry $\left.A r C\right), 136.0 \quad$ (4ry $\left.\left.A r C\right), 139.3(\mathrm{EtCH}=\mathrm{CH})\right), 145.2$ $(\mathrm{C}(4) \operatorname{ArC}(1)), 165.8(C(2)=\mathrm{O}) ; \mathrm{m} / z(\mathrm{NSI}) 356\left([\mathrm{M}+\mathrm{H}]^{+}, 37 \%\right) ; \mathrm{HRMS}(\mathrm{NSI}) \mathrm{C}_{20} \mathrm{H}_{22} \mathrm{NO}_{3} \mathrm{~S}^{+}\left([\mathrm{M}+\mathrm{H}]^{+}\right)$ requires 356.1315 ; found $356.1316(+0.3 \mathrm{ppm})$.

Data for lactam $(3 S, 4 S)-76$ : $\mathrm{mp} 85-87^{\circ} \mathrm{C}$; Chiral HPLC Chiralpak AD-H (10\% IPA:hexane, flow rate 1 $\left.\mathrm{mL} \mathrm{min}^{-1}, 211 \mathrm{~nm}, 30{ }^{\circ} \mathrm{C}\right) \mathrm{t}_{\mathrm{R}}(3 S, 4 S): 14.9 \mathrm{~min}, \mathrm{t}_{\mathrm{R}}(3 R, 4 R): 27.9 \mathrm{~min}, 74 \%$ ee; $v_{\max }(\mathrm{ATR}) / \mathrm{cm}^{-1} 2967(\mathrm{C}-\mathrm{H})$, $1788(\mathrm{C}=\mathrm{O}), 1456,1368(\mathrm{~S}=\mathrm{O}), 1171(\mathrm{~S}=\mathrm{O})$; Data for major diastereomer: $\delta_{\mathrm{H}}\left(500 \mathrm{MHz}, \mathrm{CDCl}_{3}\right) 0.72$ (3H, t, J 7.4 Hz, $\left.\left.\mathrm{CH}_{3} \mathrm{CH}_{2}\right), 1.78-1.84\left(2 \mathrm{H}, \mathrm{m}, \mathrm{CH}_{3} \mathrm{CH}_{2}\right), 2.45(3 \mathrm{H}, \mathrm{s}, \mathrm{ArCH})_{3}\right), 4.16(1 \mathrm{H}, \mathrm{ddd}, J$ 7.6, 6.7, 1.0, C(3)H), $4.76(1 \mathrm{H}, \mathrm{ddt}, J 15.5,7.6,1.6 \mathrm{~Hz}, \mathrm{EtCH}=\mathrm{CH}), 5.24(1 \mathrm{H}, \mathrm{d}, J 6.7 \mathrm{~Hz}, \mathrm{C}(4) H), 5.69(1 \mathrm{H}, \mathrm{dtd}, J$ 15.5, 6.4, $1.2 \mathrm{~Hz}, \mathrm{EtCH}=\mathrm{CH}), 7.08-7.11(2 \mathrm{H}, \mathrm{m}, \operatorname{Ar} H), 7.25-7.31(5 \mathrm{H}, \mathrm{m}, \operatorname{Ar} H), 7.76-7.78(2 \mathrm{H}, \mathrm{m}$, $\left.\mathrm{SO}_{2} \operatorname{Ar}(2,6) H\right) ; \delta_{\mathrm{C}}\left(100 \mathrm{MHz}, \mathrm{CDCl}_{3}\right) 13.1\left(\mathrm{CH}_{3} \mathrm{CH}_{2}\right), 21.8\left(\mathrm{ArCH}_{3}\right), 25.6\left(\mathrm{CH}_{3} \mathrm{CH}_{2}\right), 58.2(C(3), 61.8$ $(C(4)), 117.7(\mathrm{EtCH}=C H), 127.5(\mathrm{C}(4) \operatorname{ArC}(2,6)), 127.8\left(\mathrm{SO}_{2} \operatorname{ArC}(2,6)\right), 128.4(\mathrm{C}(4) \operatorname{ArC}(3,5)), 128.6$ (C(4)ArC(4)), $130.0 \quad\left(\mathrm{SO}_{2} \operatorname{ArC}(3.5)\right), 134.0 \quad$ (4ry $\left.\left.A r C\right), 135.8 \quad(4 \mathrm{ry} A r C), 140.0(\mathrm{EtCH}=\mathrm{CH})\right), 145.4$ (C(4) $\operatorname{ArC}(1)), 165.7(C(2)=\mathrm{O}) ; m / z(\mathrm{NSI}) 356\left([\mathrm{M}+\mathrm{H}]^{+}, 39 \%\right) ; \mathrm{HRMS}(\mathrm{NSI}) \mathrm{C}_{20} \mathrm{H}_{22} \mathrm{NO}_{3} \mathrm{~S}^{+}\left([\mathrm{M}+\mathrm{H}]^{+}\right)$ requires 356.1315 ; found $356.1316(+0.3 \mathrm{ppm})$

Reaction carried out for $1.5 \mathrm{~h}$ at $-78{ }^{\circ} \mathrm{C}$ gave crude lactams (3S,4R)-32:(3S,4S)-76 (82:18 dr). Chromatographic purification (eluent $\mathrm{Et}_{2} \mathrm{O}$ :petrol 20:80) gave lactam $(3 S, 4 R)-32(>98: 2 \mathrm{dr})$ as a colourless oil (189 mg, 67\%) with identical spectroscopic properties as before in $98 \% e e ;[\alpha]_{D}^{20}+2.4\left(c 0.5, \mathrm{CH}_{2} \mathrm{Cl}_{2}\right)$ and lactam $(3 S, 4 S)-76(98: 2 \mathrm{dr})$ as a white solid $(47.0 \mathrm{mg}, 16 \%)$ with identical spectroscopic properties as before in $>99 \%$ ee; $[\alpha]_{D}^{20}-9.3\left(c 0.5, \mathrm{CH}_{2} \mathrm{Cl}_{2}\right)$.

\section{$(3 S, 4 R)-1-[(4-m e t h y l b e n z e n e) s u l f o n y l]-3-[(1 E)-3-m e t h y l b u t-1-e n-1-y l]-4-p h e n y l a z e t i d i n-2-o n e ~ 33$}

Following general procedure A, (E)-5-methylhex-3-enoic acid 65 (205 mg, 1.60 mmol), $i \operatorname{Pr}_{2} \mathrm{NEt}(420 \mu \mathrm{L}$, $2.40 \mathrm{mmol})$ and pivaloyl chloride $(296 \mu \mathrm{L}, 2.40 \mathrm{mmol})$ in $\mathrm{CH}_{2} \mathrm{Cl}_{2}(10 \mathrm{~mL}), \mathrm{HBTM}-2.1(2 S, 3 R)-\mathbf{2 3}(24.6$ 
mg, $0.08 \mathrm{mmol}, 10 \mathrm{~mol} \%$ ), imine $19(207 \mathrm{mg}, 0.80 \mathrm{mmol})$ and $i \operatorname{Pr}_{2} \mathrm{NEt}(348 \mu \mathrm{L}, 2.00 \mathrm{mmol})$ for $1.5 \mathrm{~h}$ at $\mathrm{rt}$ gave crude lactam $(3 S, 4 R)-33(73: 27 \mathrm{dr})$. Chromatographic purification (eluent $\mathrm{Et}_{2} \mathrm{O}$ :petrol 15:85) gave lactam (3S,4R)-33 (>98:2 dr) as a colourless solid (155 mg, 53\%); Chiral HPLC Chiralpak AD-H (10\% IPA:hexane, flow rate $\left.1 \mathrm{~mL} \min ^{-1}, 220 \mathrm{~nm}, 30^{\circ} \mathrm{C}\right) \mathrm{t}_{\mathrm{R}}(3 S, 4 R): 9.9 \mathrm{~min}, \mathrm{t}_{\mathrm{R}}(3 R, 4 S): 14.4 \mathrm{~min}, 82 \%$ ee; $v_{\max }$ $(\mathrm{ATR}) / \mathrm{cm}^{-1} 2961(\mathrm{C}-\mathrm{H}), 1794(\mathrm{C}=\mathrm{O}), 1597,1435,1366(\mathrm{~S}=\mathrm{O}), 1169(\mathrm{~S}=\mathrm{O})$; Data for major diastereomer: $\delta_{\mathrm{H}}\left(500 \mathrm{MHz}, \mathrm{CDCl}_{3}\right) 0.95\left(6 \mathrm{H}, \mathrm{d}, J 6.8 \mathrm{~Hz}, \mathrm{CH}\left(\mathrm{CH}_{3}\right)_{2}\right), 2.23-2.30\left(1 \mathrm{H}, \mathrm{m}, \mathrm{CH}\left(\mathrm{CH}_{3}\right)_{2}\right), 2.42(3 \mathrm{H}, \mathrm{s}$, $\left.\mathrm{ArCH}_{3}\right), 3.68-3.70(1 \mathrm{H}, \mathrm{m}, \mathrm{C}(3) H), 4.77(1 \mathrm{H}, \mathrm{d}, J 3.3 \mathrm{~Hz}, \mathrm{C}(4) H), 5.38(1 \mathrm{H}, \mathrm{ddd}, J 15.5,7.7,1.4 \mathrm{~Hz}$, $i \operatorname{PrCH}=\mathrm{CH}), 5.62(1 \mathrm{H}, \mathrm{ddd}, J 15.5,6.5,1.1 \mathrm{~Hz}, i \operatorname{PrCH}=\mathrm{CH}), 7.21-7.25(4 \mathrm{H}, \mathrm{m}, \mathrm{ArH}), 7.27-7.34(3 \mathrm{H}, \mathrm{m}$, $\operatorname{ArH}), 7.62-7.64\left(2 \mathrm{H}, \mathrm{m}, \mathrm{SO}_{2} \mathrm{Ar}(2,6) H\right) ; \delta_{\mathrm{C}}\left(125 \mathrm{MHz}, \mathrm{CDCl}_{3}\right) 21.8\left(\mathrm{ArCH}_{3}\right), 22.0\left(\mathrm{CH}\left(\mathrm{CH}_{3}\right)_{2}\right), 31.2$ $\left(\mathrm{CH}\left(\mathrm{CH}_{3}\right)_{2}\right), 62.5(C(3)), 64.3(C(4)), 117.3(i \mathrm{PrCH}=C \mathrm{H}), 126.7(\mathrm{C}(4) \operatorname{ArC}(2,6)), 127.6\left(\mathrm{SO}_{2} \operatorname{ArC}(2,6)\right)$, $128.9(\mathrm{C}(4) \operatorname{ArC}(3,5)), 129.0(\mathrm{C}(4) \operatorname{ArC}(4)), 129.8\left(\mathrm{SO}_{2} \operatorname{ArC}(3,5)\right), 135.7$ (4ry $\left.A r C\right), 136.0$ (4ry $\left.\operatorname{ArC}\right), 144.4$ $(i \mathrm{PrCH}=\mathrm{CH}), 145.2(\mathrm{C}(4) \operatorname{ArC}(1)), 165.8 \quad(C(2)=\mathrm{O}) ; m / z \quad(\mathrm{NSI}) 370\left([\mathrm{M}+\mathrm{H}]^{+}, 32 \%\right) ; \mathrm{HRMS}$ (NSI) $\mathrm{C}_{21} \mathrm{H}_{24} \mathrm{NO}_{3} \mathrm{~S}^{+}\left([\mathrm{M}+\mathrm{H}]^{+}\right)$requires 370.1471 ; found $370.1472(+0.2 \mathrm{ppm})$.

Reaction carried out for $1.5 \mathrm{~h}$ at $-78{ }^{\circ} \mathrm{C}$ gave crude lactam $(3 S, 4 R)-33(79: 21 \mathrm{dr})$. Chromatographic purification (eluent $\mathrm{Et}_{2} \mathrm{O}$ :petrol 15:85) gave lactam $(3 S, 4 R)-33(>98: 2 \mathrm{dr})$ as a colourless oil (185 $\mathrm{mg}$, $63 \%$ ) with identical spectroscopic properties as before in $97 \%$ ee; $[\alpha]_{D}^{20}+1.6\left(c 0.5, \mathrm{CH}_{2} \mathrm{Cl}_{2}\right)$.

(3S,4R)-1-[(4-methylbenzene)sulfonyl]-4-phenyl-3-[(1E)-3-phenylprop-1-en-1-yl]azetidin-2-one 34

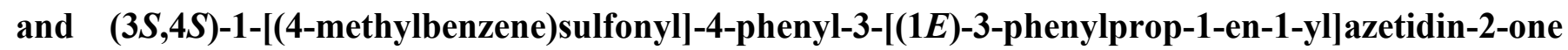
77

Following general procedure A, (E)-5-phenylpent-3-enoic acid 66 (282 mg, $1.60 \mathrm{mmol}), i \operatorname{Pr}_{2} \mathrm{NEt}(420 \mu \mathrm{L}$, $2.40 \mathrm{mmol})$ and pivaloyl chloride $(296 \mu \mathrm{L}, 2.40 \mathrm{mmol})$ in $\mathrm{CH}_{2} \mathrm{Cl}_{2}(10 \mathrm{~mL}), \mathrm{HBTM}-2.1(2 S, 3 R)-23$ (24.6 mg, $0.08 \mathrm{mmol}, 10 \mathrm{~mol} \%$ ), imine $19(207 \mathrm{mg}, 0.80 \mathrm{mmol})$ and $i \operatorname{Pr}_{2} \mathrm{NEt}(348 \mu \mathrm{L}, 2.00 \mathrm{mmol})$ for $1.5 \mathrm{~h}$ at $\mathrm{rt}$ gave crude lactams $(3 S, 4 R)-34$ and $(3 S, 4 S)-77$ (72:28 dr). Chromatographic purification (eluent 
$\mathrm{Et}_{2} \mathrm{O}$ :petrol 25:75) gave lactam $(3 S, 4 R)-34(>98: 2 \mathrm{dr})$ as a colourless oil (197 $\left.\mathrm{mg}, 59 \%\right)$ and lactam $(3 S, 4 S)-77(95: 5 \mathrm{dr})$ as a colourless oil $(61 \mathrm{mg}, 18 \%)$ :

Data for lactam $(3 S, 4 R)-34$ : Chiral HPLC Chiralpak AD-H (10\% IPA:hexane, flow rate $1 \mathrm{~mL} \mathrm{~min}^{-1}, 211$ $\left.\mathrm{nm}, 30{ }^{\circ} \mathrm{C}\right) \mathrm{t}_{\mathrm{R}}(3 S, 4 R): 18.4 \mathrm{~min}, \mathrm{t}_{\mathrm{R}}(3 R, 4 S): 25.3 \mathrm{~min}, 62 \% e e ; v_{\max }(\mathrm{ATR}) / \mathrm{cm}^{-1} 3028(\mathrm{C}-\mathrm{H}), 1792(\mathrm{C}=\mathrm{O})$, 1597, $1364(\mathrm{~S}=\mathrm{O}), 1169(\mathrm{~S}=\mathrm{O})$; Data for major diastereoisomer: $\delta_{\mathrm{H}}\left(500 \mathrm{MHz}, \mathrm{CDCl}_{3}\right) 2.42(3 \mathrm{H}, \mathrm{s}$, $\left.\mathrm{ArCH}_{3}\right), 3.37\left(2 \mathrm{H}\right.$, d. $\left.J 6.7 \mathrm{~Hz}, \mathrm{PhCH}_{2}\right), 3.76(1 \mathrm{H}, \mathrm{ddd}, J$ 7.7, 3.3, $0.9 \mathrm{~Hz}, \mathrm{C}(3) H), 4.81(1 \mathrm{H}, \mathrm{d}, J 3.3 \mathrm{~Hz}$, $\mathrm{C}(4) H), 5.51(1 \mathrm{H}, \mathrm{ddt}, J 15.4,7.7,1.5 \mathrm{~Hz}, \mathrm{BnCH}=\mathrm{CH}), 5.84(1 \mathrm{H}, \mathrm{dtd}, J 15.3,6.7,1.2 \mathrm{~Hz}, \mathrm{BnCH}=\mathrm{CH})$, 7.12-7.14 (2H, m, ArH), 7.20-7.23 (5H, m, ArH), 7.28-7.35 (5H, m, ArH), 7.62-7.64 (2H, m, $\left.\mathrm{SO}_{2}(2,6) H\right)$; $\delta_{\mathrm{C}}\left(125 \mathrm{MHz}, \mathrm{CDCl}_{3}\right) 21.8\left(\mathrm{ArCH}_{3}\right), 38.9\left(\mathrm{PhCH}_{2} \mathrm{CH}=\mathrm{CH}\right), 62.3(\mathrm{C}(3)), 64.1(C(4)), 121.4(\mathrm{BnCH}=\mathrm{CH})$, $126.4(\mathrm{ArC}), 126.7(\mathrm{ArC}), 127.5(\mathrm{ArC}), 128.6(\mathrm{ArC}), 128.6(\mathrm{ArC}), 128.9(\mathrm{ArC}), 129.1(\mathrm{C}(4) \operatorname{ArC}(4)), 129.8$ $\left(\mathrm{SO}_{2} \operatorname{ArC}(3,5)\right), 135.7$ (4ry $\left.A r C\right), 135.8$ (4ry $\left.A r C\right), 136.1 \quad(\mathrm{BnCH}=\mathrm{CH}), 139.0 \quad\left(\mathrm{SO}_{2} \operatorname{ArC}(1)\right), 145.2$ $(\mathrm{C}(4) \operatorname{ArC}(1)), 165.4(C(2)=\mathrm{O}) ; m / z(\mathrm{NSI}) 418\left([\mathrm{M}+\mathrm{H}]^{+}, 20 \%\right) ; \mathrm{HRMS}(\mathrm{NSI}) \mathrm{C}_{25} \mathrm{H}_{24} \mathrm{NO}_{3} \mathrm{~S}^{+}\left([\mathrm{M}+\mathrm{H}]^{+}\right)$ requires 418.1471 ; found $418.1467(-1.1 \mathrm{ppm})$.

Data for lactam $(3 S, 4 S)-77$ : Chiral HPLC Chiralpak AD-H (10\% IPA:hexane, flow rate $1 \mathrm{~mL} \mathrm{~min}^{-1}, 211$ $\left.\mathrm{nm}, 30{ }^{\circ} \mathrm{C}\right) \mathrm{t}_{\mathrm{R}}(3 S, 4 S): 14.4 \mathrm{~min}, \mathrm{t}_{\mathrm{R}}(3 R, 4 R): 26.7 \mathrm{~min}, 39 \% e e ; v_{\max }(\mathrm{ATR}) / \mathrm{cm}^{-1} 3028,2924(\mathrm{C}-\mathrm{H}), 1788$ $(\mathrm{C}=\mathrm{O}), 1595,1359(\mathrm{~S}=\mathrm{O}), 1167(\mathrm{~S}=\mathrm{O})$; Data for minor diastereoisomer: $\delta_{\mathrm{H}}\left(500 \mathrm{MHz}, \mathrm{CDCl}_{3}\right) 2.45(3 \mathrm{H}$, s, $\left.\mathrm{ArCH}_{3}\right), 3.12\left(2 \mathrm{H}, \mathrm{d} . J 6.7 \mathrm{~Hz}, \mathrm{PhCH}_{2}\right), 4.18-4.21(1 \mathrm{H}, \mathrm{m}, \mathrm{C}(3) H), 4.85(1 \mathrm{H}, \mathrm{ddt}, J 15.4,7.7,1.5 \mathrm{~Hz}$, $\mathrm{BnCH}=\mathrm{CH}), 5.26(1 \mathrm{H}, \mathrm{d}, J 6.7 \mathrm{~Hz}, \mathrm{C}(4) H), 5.78(1 \mathrm{H}, \mathrm{dtd}, J 15.4,6.8,1.2, \mathrm{BnCH}=\mathrm{CH}), 6.76-6.79(2 \mathrm{H}, \mathrm{m}$, $\operatorname{Ar} H), 7.08-7.23(5 \mathrm{H}, \mathrm{m}, \operatorname{Ar} H), 7.27-7.35(5 \mathrm{H}, \mathrm{m}, \operatorname{Ar} H), 7.77-7.79\left(2 \mathrm{H}, \mathrm{m}, \mathrm{SO}_{2}(2,6) H\right) ; \delta_{\mathrm{C}}(125 \mathrm{MHz}$, $\left.\mathrm{CDCl}_{3}\right) 21.9\left(\mathrm{ArCH}_{3}\right), 38.9\left(\mathrm{PhCH}_{2}\right), 58.1(\mathrm{C}(3)), 61.8(C(4)), 120.4(\mathrm{BnCH}=C \mathrm{H}), 126.2(\mathrm{ArC}), 127.4$ (ArC), $127.8(A r C), 128.4(A r C), 128.6(A r C), 128.7(A r C), 128.7(A r C), 130.0\left(\mathrm{SO}_{2} A r C(3,5)\right), 134.0(4 \mathrm{ry}$ $A r C), 135.7(4 \mathrm{ry} A r C), 136.8(\mathrm{BnCH}=\mathrm{CH}), 138.9\left(\mathrm{SO}_{2} \operatorname{ArC}(1)\right), 145.5(\mathrm{C}(4) \operatorname{ArC}(1)), 165.4(C(2)=\mathrm{O}) ; m / z$ (NSI) $418\left([\mathrm{M}+\mathrm{H}]^{+}, 28 \%\right)$; HRMS (NSI) $\mathrm{C}_{25} \mathrm{H}_{24} \mathrm{NO}_{3} \mathrm{~S}^{+}\left([\mathrm{M}+\mathrm{H}]^{+}\right)$requires 418.1471; found $418.1459(-3.0$ ppm) 
Reaction carried out for $1.5 \mathrm{~h}$ at $-78{ }^{\circ} \mathrm{C}$ gave crude lactams (3S,4R)-34:(3S,4S)-77 (72:28 dr). Chromatographic purification (eluent $\mathrm{Et}_{2} \mathrm{O}$ :petrol 25:75) gave lactam $(3 S, 4 R)-34(>98: 2 \mathrm{dr})$ as a colourless oil (189 mg, 57\%) with identical spectroscopic properties as before in $95 \% e e ;[\alpha]_{D}^{20}+0.8\left(c 0.5, \mathrm{CH}_{2} \mathrm{Cl}_{2}\right)$ and lactam $(3 S, 4 S)-77(95: 5 \mathrm{dr})$ as a colourless oil $(53.0 \mathrm{mg}, 16 \%)$ with identical spectroscopic properties as before in $97 \%$ ee; $[\alpha]_{D}^{20}-6.8\left(c 0.5, \mathrm{CH}_{2} \mathrm{Cl}_{2}\right)$.

(3S,4R)-1-[(4-methylbenzene)sulfonyl]-4-phenyl-3-[(1Z)-prop-1-en-1-yl]azetidin-2-one $35 \quad$ and $(3 S, 4 S)-1-[(4-m e t h y l b e n z e n e) s u l f o n y l]-4-p h e n y l-3-[(1 Z)-p r o p-1-e n-1-y l]$ azetidin-2-one 78

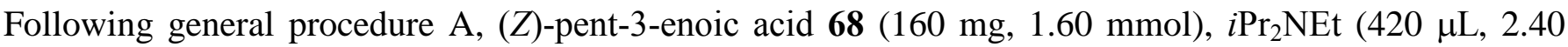
mmol) and pivaloyl chloride $(296 \mu \mathrm{L}, 2.40 \mathrm{mmol})$ in $\mathrm{CH}_{2} \mathrm{Cl}_{2}(10 \mathrm{~mL}), \mathrm{HBTM}-2.1(2 S, 3 R)-23$ (24.6 mg, $0.08 \mathrm{mmol}, 10 \mathrm{~mol} \%)$, imine $19(207 \mathrm{mg}, 0.80 \mathrm{mmol})$ and $i \operatorname{Pr}_{2} \mathrm{NEt}(348 \mu \mathrm{L}, 2.00 \mathrm{mmol})$ for $1.5 \mathrm{~h}$ at $\mathrm{rt}$ gave crude lactams $(3 S, 4 R)-35$ and $(3 S, 4 S)-78$ (48:52 dr). Chromatographic purification (eluent $\mathrm{Et}_{2} \mathrm{O}$ :petrol 20:80) gave lactam $(3 S, 4 R)-35(92: 8 \mathrm{dr})$ as a colourless oil $(89 \mathrm{mg}, 33 \%)$ and lactam $(3 S, 4 S)-$ $78(88: 12 \mathrm{dr})$ as a white solid (97 $\mathrm{mg}, 36 \%)$ :

Data for lactam $(3 S, 4 R)-35$ : Chiral HPLC Chiralpak AD-H (10\% IPA:hexane, flow rate $1 \mathrm{~mL} \mathrm{~min}^{-1}, 211$ $\left.\mathrm{nm}, 30^{\circ} \mathrm{C}\right) \mathrm{t}_{\mathrm{R}}(3 R, 4 S): 14.5 \mathrm{~min}, \mathrm{t}_{\mathrm{R}}(3 S, 4 R): 16.0 \mathrm{~min}, 92 \% e e ; v_{\max }(\mathrm{ATR}) / \mathrm{cm}^{-1} 3030,2967(\mathrm{C}-\mathrm{H}), 1788$ $(\mathrm{C}=\mathrm{O}), 1456,1362(\mathrm{~S}=\mathrm{O}), 1167(\mathrm{~S}=\mathrm{O})$; Data for major diastereoisomer: $\delta_{\mathrm{H}}\left(500 \mathrm{MHz}, \mathrm{CDCl}_{3}\right) 1.51(3 \mathrm{H}$, dd, J 6.9, $\left.1.8 \mathrm{~Hz}, \mathrm{CH}_{3} \mathrm{CH}=\mathrm{CH}\right), 2.43\left(3 \mathrm{H}, \mathrm{s}, \mathrm{ArCH}_{3}\right), 4.00-4.02(1 \mathrm{H}, \mathrm{m}, \mathrm{C}(3) H), 4.75(1 \mathrm{H}, \mathrm{d}, J 3.2 \mathrm{~Hz}$, $\mathrm{C}(4) H), 5.44\left(1 \mathrm{H}, \mathrm{ddq}, J 10.6,8.8,1.8 \mathrm{~Hz}, \mathrm{CH}_{3} \mathrm{CH}=\mathrm{CH}\right), 5.78(1 \mathrm{H}, \mathrm{dqd}, J$ 10.7, 6.9, $1.4 \mathrm{~Hz}$, $\left.\mathrm{CH}_{3} \mathrm{CH}=\mathrm{CH}\right), 7.23-7.25(4 \mathrm{H}, \mathrm{m}, \mathrm{Ar} H), 7.28-7.35(3 \mathrm{H}, \mathrm{m}, \mathrm{Ar} H), 7.62-7.65\left(2 \mathrm{H}, \mathrm{m}, \mathrm{SO}_{2} \operatorname{Ar}(2,6) H\right) ; \delta_{\mathrm{C}}(125$ $\left.\mathrm{MHz}, \mathrm{CDCl}_{3}\right) 14.0\left(\mathrm{CH}_{3} \mathrm{CH}=\mathrm{CH}\right), 21.8\left(\mathrm{ArCH}_{3}\right), 58.2(C(3)), 64.4(C(4)), 120.5\left(\mathrm{CH}_{3} \mathrm{CH}=C \mathrm{H}\right), 126.7$ $(\mathrm{C}(4) \operatorname{ArC}(3,5)), 127.6\left(\mathrm{SO}_{2} \operatorname{ArC}(3,5)\right), 128.9\left(\mathrm{SO}_{2} \operatorname{ArC}(2,6)\right), 129.1(\mathrm{C}(4) \operatorname{ArC}(4)), 129.9(\mathrm{C}(4) \operatorname{ArC}(2,6))$, $132.1\left(\mathrm{CH}_{3} \mathrm{CH}=\mathrm{CH}\right), 135.7$ (4ry $\left.A r C\right), 136.0$ (4ry $\left.A r C\right), 145.3(\mathrm{C}(4) A r C(1)), 165.9(C(2)=\mathrm{O}) ; m / z(\mathrm{NSI})$ $342\left([\mathrm{M}+\mathrm{H}]^{+}, 42 \%\right)$; HRMS (NSI) $\mathrm{C}_{19} \mathrm{H}_{20} \mathrm{NO}_{3} \mathrm{~S}^{+}\left([\mathrm{M}+\mathrm{H}]^{+}\right)$requires 342.1158; found $342.1159(+0.2$ ppm) 
Data for lactam $(3 S, 4 S)-78: \mathrm{mp} 83-85^{\circ} \mathrm{C}$ : Chiral HPLC Chiralcel OD-H (10\% IPA:hexane, flow rate $1 \mathrm{~mL}$ $\left.\min ^{-1}, 211 \mathrm{~nm}, 30^{\circ} \mathrm{C}\right) \mathrm{t}_{\mathrm{R}}(3 S, 4 S): 12.0 \mathrm{~min}, \mathrm{t}_{\mathrm{R}}(3 R, 4 R): 15.8 \mathrm{~min}, 98 \%$ ee; $v_{\max }(\mathrm{ATR}) / \mathrm{cm}^{-1} 3032,2922(\mathrm{C}-$ $\mathrm{H}), 1788(\mathrm{C}=\mathrm{O}), 1458,1354(\mathrm{~S}=\mathrm{O}), 1165(\mathrm{~S}=\mathrm{O})$; Data for minor diastereoisomer: $\delta_{\mathrm{H}}\left(500 \mathrm{MHz}, \mathrm{CDCl}_{3}\right)$ 1.55-1.57 (3H, m, $\left.\mathrm{CH}_{3} \mathrm{CH}=\mathrm{CH}\right), 2.46\left(3 \mathrm{H}, \mathrm{s}, \mathrm{ArCH}_{3}\right), 4.47-4.50(1 \mathrm{H}, \mathrm{m}, \mathrm{C}(3) H), 4.89-4.94(1 \mathrm{H}, \mathrm{m}$, $\left.\mathrm{CH}_{3} \mathrm{CH}=\mathrm{CH}\right), 5.31(1 \mathrm{H}, \mathrm{d}, J 6.8 \mathrm{~Hz}, \mathrm{C}(4) H), 5.54\left(1 \mathrm{H}, \mathrm{dqd}, J 10.8,6.9,1.5 \mathrm{~Hz}, \mathrm{CH}_{3} \mathrm{CH}=\mathrm{CH}\right), 7.09-7.11$ $(2 \mathrm{H}, \mathrm{m}, \operatorname{Ar} H), 7.23-7.34(5 \mathrm{H}, \mathrm{m}, \mathrm{Ar} H), 7.76-7.78\left(2 \mathrm{H}, \mathrm{m}, \mathrm{SO}_{2} \mathrm{Ar}(2,6) H\right) ; \delta_{\mathrm{C}}\left(125 \mathrm{MHz}, \mathrm{CDCl}_{3}\right) 13.9$ $\left(\mathrm{CH}_{3} \mathrm{CH}=\mathrm{CH}\right), 21.9\left(\mathrm{ArCH}_{3}\right), 53.7(C(3)), 61.8(C(4)), 118.6\left(\mathrm{CH}_{3} \mathrm{CH}=\mathrm{CH}\right), 127.4(\mathrm{C}(4) \operatorname{ArC}(3,5)), 127.8$ $\left(\mathrm{SO}_{2} \operatorname{ArC}(3,5)\right), 128.5\left(\mathrm{SO}_{2} \operatorname{ArC}(2,6)\right), 128.7(\mathrm{C}(4) \operatorname{ArC}(4)), 130.0(\mathrm{C}(4) \operatorname{ArC}(2,6)), 132.1 \quad\left(\mathrm{CH}_{3} C \mathrm{H}=\mathrm{CH}\right)$, 134.0 (4ry $A r C), 135.7$ (4ry $A r C), 145.4$ (C(4)ArC(1)), $165.9(C(2)=\mathrm{O}) ; m / z(\mathrm{NSI}) 342\left([\mathrm{M}+\mathrm{H}]^{+}, 30 \%\right)$; HRMS (NSI) $\mathrm{C}_{19} \mathrm{H}_{20} \mathrm{NO}_{3} \mathrm{~S}^{+}\left([\mathrm{M}+\mathrm{H}]^{+}\right)$requires 342.1158; found $342.1160(+0.5 \mathrm{ppm})$

Reaction carried out for $1.5 \mathrm{~h}$ at $-78{ }^{\circ} \mathrm{C}$ gave crude lactams (3S,4R)-35:(3S,4S)-78 (43:57 dr). Chromatographic purification (eluent $\mathrm{Et}_{2} \mathrm{O}$ :petrol 20:80) gave lactam $(3 S, 4 R)-35(92: 8 \mathrm{dr})$ as a colourless oil (58 mg, 21\%) with identical spectroscopic properties as before in $99 \% e e ;[\alpha]_{D}^{20}+4.2\left(c 0.5, \mathrm{CH}_{2} \mathrm{Cl}_{2}\right)$ and lactam $(3 S, 4 S)-78(88: 12 \mathrm{dr})$ as a white solid $(99.0 \mathrm{mg}, 36 \%)$ with identical spectroscopic properties as before in $99 \% e e ;[\alpha]_{D}^{20}-14.2\left(c 0.5, \mathrm{CH}_{2} \mathrm{Cl}_{2}\right)$.

\section{(3S,4R)-4-phenyl-3-((E)-prop-1-en-1-yl)-6-(trifluoromethyl)-3,4-dihydro-2H-pyran-2-one 36}

Following general procedure A, (E)-pent-3-enoic acid $24(40.6 \mu \mathrm{L}, 0.40 \mathrm{mmol}), i \mathrm{Pr}_{2} \mathrm{NEt}(104 \mu \mathrm{L}, 0.60$ mmol) and pivaloyl chloride $(74.0 \mu \mathrm{L}, 0.60 \mathrm{mmol})$ in $\mathrm{CH}_{2} \mathrm{Cl}_{2}(2 \mathrm{~mL}), \mathrm{HBTM}-2.1(2 S, 3 R)-23(6.16 \mathrm{mg}$, $0.02 \mathrm{mmol}, 5 \mathrm{~mol} \%$ ), (E)-1,1,1-trifluoro-4-phenyl-3-buten-2-one 57 (80.0 mg, $0.40 \mathrm{mmol})$ and $i \mathrm{Pr}_{2} \mathrm{NEt}$ $(174 \mu \mathrm{L}, 1.0 \mathrm{mmol})$ for 5 minutes at $\mathrm{rt}$ gave crude lactone $(3 S, 4 R)-36(88: 12 \mathrm{dr})$. Chromatographic purification (eluent $\mathrm{Et}_{2} \mathrm{O}$ :petrol 4:96) gave lactone $(3 S, 4 R)-36(88: 12 \mathrm{dr})$ as a colourless oil $(89.8 \mathrm{mg}$, 80\%); $[\alpha]_{D}^{20}-212.4\left(c\right.$ 0.5, $\left.\mathrm{CH}_{2} \mathrm{Cl}_{2}\right)$; Chiral HPLC Chiralcel OD-H (1\% IPA:hexane, flow rate $1 \mathrm{~mL} \mathrm{~min}^{-1}$, $\left.211 \mathrm{~nm}, 30{ }^{\circ} \mathrm{C}\right)$ major diastereoisomer: $\mathrm{t}_{\mathrm{R}}(3 S, 4 R): 9.7 \mathrm{~min}, \mathrm{t}_{\mathrm{R}}(3 R, 4 S): 13.2 \mathrm{~min}, 96 \%$ ee; minor diastereoisomer $\mathrm{t}_{\mathrm{R}}: 10.7 \mathrm{~min}, \mathrm{t}_{\mathrm{R}}: 14.8 \mathrm{~min}, 15 \% e e ; v_{\max }(\mathrm{ATR}) / \mathrm{cm}^{-1} 3060,3027(\mathrm{C}-\mathrm{H}), 1784(\mathrm{C}=\mathrm{O})$, 
1699; Data for major diastereoisomer: $\delta_{\mathrm{H}}\left(500 \mathrm{MHz}^{\mathrm{CDCl}} \mathrm{CD}_{3}\right) 1.68\left(3 \mathrm{H}, \mathrm{t}, J, 5.9, \mathrm{CH}_{3}\right), 3.43(1 \mathrm{H}, \mathrm{t}, J 6.9$, $\mathrm{C}(3) H), 3.70-3.75(1 \mathrm{H}, \mathrm{m}, \mathrm{C}(4) H), 5.44-5.56\left(2 \mathrm{H}, \mathrm{m}, \mathrm{C}(3) \mathrm{CH}=\mathrm{CHCH}_{3}\right.$ and $\left.\mathrm{C}(3) \mathrm{CH}=\mathrm{CHCH}_{3}\right), 6.09(1 \mathrm{H}$, $\mathrm{d}, J 4.5, \mathrm{C}(5) H), 7.11(2 \mathrm{H}, \mathrm{d}, J 7.8, \mathrm{C}(4) \operatorname{Ar}(2,6) H), 7.31-7.40(3 \mathrm{H}, \mathrm{m}, \mathrm{C}(4) \operatorname{Ar}(3,5) H$ and $\mathrm{C}(4) \operatorname{Ar}(4) H) ; \delta_{\mathrm{C}}$ $\left(75 \mathrm{MHz}, \mathrm{CDCl}_{3}\right) 18.1\left(C_{3}\right), 43.2(C(4)), 49.9(C(3)), 109.7(\mathrm{q}, J 3.5, C(5)), 118.5\left(\mathrm{q}, J 270, C \mathrm{~F}_{3}\right), 123.8$ $\left(\mathrm{C}(3) \mathrm{CH}=\mathrm{CHCH}_{3}\right), 127.4(A r C), 128.1(A r C), 129.2(A r C), 132.1 \quad\left(\mathrm{C}(3) \mathrm{CH}=C \mathrm{HCH}_{3}\right), 138.7$ (4ry $\mathrm{C}(4) \operatorname{ArC}(1)), 140.8(\mathrm{q}, J 37.9, C(6)), 166.1(C(2)) ; \delta_{\mathrm{F}}\left(376 \mathrm{MHz}, \mathrm{CDCl}_{3}\right)-72.6\left(\mathrm{CF}_{3}\right)$; Selected data or minor diastereoisomer: $\delta_{\mathrm{H}}\left(500 \mathrm{MHz}, \mathrm{CDCl}_{3}\right) 3.63(1 \mathrm{H}, \mathrm{t}, J$ 7.8, $\mathrm{C}(3) H), 3.86-3.88(1 \mathrm{H}, \mathrm{m}, \mathrm{C}(4) H), 5.13$ $\left(1 \mathrm{H}, \mathrm{dd}, J 15.4,8.6, \mathrm{C}(3) \mathrm{CH}=\mathrm{CHCH}_{3}\right), 5.69\left(1 \mathrm{H}, \mathrm{dq}, J 14.7,7.1, \mathrm{C}(3) \mathrm{CH}=\mathrm{CHCH}_{3}\right), 6.23(1 \mathrm{H}, \mathrm{d}, J 5.7$, $\mathrm{C}(5) H), 7.11(2 \mathrm{H}, \mathrm{d}, J$ 7.7, $\operatorname{Ar}(2,6) H) ; \delta_{\mathrm{C}}\left(75 \mathrm{MHz} \mathrm{CDCl}_{3}\right) 18.0\left(\mathrm{CH}_{3}\right), 43.0(C(4)), 47.7(C(3)), 110.8(\mathrm{q}$, $J$ 3.5, $C(5)), 122.6\left(\mathrm{C}(3) C H=\mathrm{CHCH}_{3}\right), 128.2(\mathrm{ArC}), 128.3(\mathrm{ArC}), 129.1(\mathrm{ArC}), 132.2\left(\mathrm{C}(3) \mathrm{CH}=C \mathrm{HCH}_{3}\right)$, $166.6(C(2)) ; \delta_{\mathrm{F}}\left(376 \mathrm{MHz}, \mathrm{CDCl}_{3}\right)-72.7\left(\mathrm{CF}_{3}\right) ; \mathrm{m} / z\left(\mathrm{NSI}^{+}\right) 300\left(\left[\mathrm{M}+\mathrm{NH}_{4}\right]^{+}, 100 \%\right) ; \mathrm{HRMS}\left(\mathrm{NSI}^{+}\right)$ $\mathrm{C}_{15} \mathrm{H}_{17} \mathrm{~F}_{3} \mathrm{NO}_{2}^{+}\left(\left[\mathrm{M}+\mathrm{NH}_{4}\right]^{+}\right)$requires 300.1206 ; found $300.1206(+0.0 \mathrm{ppm})$.

\section{(3S,4R)-4-phenyl-3-((E)-but-1-en-1-yl)-6-(trifluoromethyl)-3,4-dihydro-2H-pyran-2-one 37}

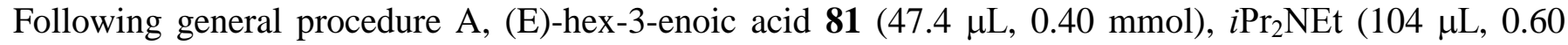
mmol) and pivaloyl chloride $(74.0 \mu \mathrm{L}, 0.60 \mathrm{mmol})$ in $\mathrm{CH}_{2} \mathrm{Cl}_{2}(2 \mathrm{~mL}), \mathrm{HBTM}-2.1(2 S, 3 R)-23$ (6.16 mg, $0.02 \mathrm{mmol}, 5 \mathrm{~mol} \%)$, (E)-1,1,1-trifluoro-4-phenyl-3-buten-2-one 57 (80.0 mg, $0.40 \mathrm{mmol})$ and $i \operatorname{Pr}_{2} \mathrm{NEt}$ $(174 \mu \mathrm{L}, 1.0 \mathrm{mmol})$ for 5 minutes at $\mathrm{rt}$ gave crude lactone $(3 S, 4 R)-37(90: 10 \mathrm{dr})$. Chromatographic purification (eluent $\mathrm{Et}_{2} \mathrm{O}$ :petrol 3:97) gave lactone $(3 \mathrm{~S}, 4 R)-37$ (93:7 $\mathrm{dr}$ ) as a colourless oil $(98.7 \mathrm{mg}, 83 \%)$; $[\alpha]_{D}^{20}-191.0\left(c\right.$ 0.5, $\left.\mathrm{CH}_{2} \mathrm{Cl}_{2}\right)$; Chiral HPLC Chiralcel OD-H (1\% IPA:hexane, flow rate $1 \mathrm{~mL} \mathrm{~min}^{-1}, 211$ $\left.\mathrm{nm}, \quad 30{ }^{\circ} \mathrm{C}\right)$ major diastereoisomer: $\mathrm{t}_{\mathrm{R}}(3 S, 4 R): 8.9 \mathrm{~min}, \mathrm{t}_{\mathrm{R}}(3 R, 4 S): 12.4 \mathrm{~min}$, 96\% ee; minor diastereoisomer $\mathrm{t}_{\mathrm{R}}: 9.9 \mathrm{~min}, \mathrm{t}_{\mathrm{R}}: 14.4 \mathrm{~min}, 12 \%$ ee; $v_{\max }(\mathrm{ATR}) / \mathrm{cm}^{-1} 3065,2968(\mathrm{C}-\mathrm{H}), 1786(\mathrm{C}=\mathrm{O}), 1699$; Data for major diastereoisomer: $\delta_{\mathrm{H}}\left(500 \mathrm{MHz}, \mathrm{CDCl}_{3}\right) 0.92\left(3 \mathrm{H}, \mathrm{t}, J\right.$ 7.5, $\left.\mathrm{CH}_{3}\right), 2.00-2.06(2 \mathrm{H}, \mathrm{m}$, $\left.\mathrm{CH}_{2} \mathrm{CH}_{3}\right), 3.42\left(1 \mathrm{H}, \mathrm{t}, J\right.$ 7.1, C(3)H), 3.71-3.74 (1H, m, C(4)H), 5.41-5.45 (1H, m, C(3)CH=CHCH${ }_{2} \mathrm{CH}_{3}$ or $\left.\mathrm{C}(3) \mathrm{CH}=\mathrm{CHCH}_{2} \mathrm{CH}_{3}\right), 5.49-5.54\left(1 \mathrm{H}, \mathrm{m}, \mathrm{C}(3) \mathrm{CH}=\mathrm{CHCH}_{2} \mathrm{CH}_{3}\right.$ or $\left.\mathrm{C}(3) \mathrm{CH}=\mathrm{CHCH}_{2} \mathrm{CH}_{3}\right), 6.10(1 \mathrm{H}, \mathrm{d}$, $J$ 4.6, C(5)H), 7.14-7.16 (2H, m, C(4)Ar(2,6)H), 7.31-7.40 (3H, m, C(4)Ar(3,5)H and $\mathrm{C}(4) \operatorname{Ar}(4) H) ; \delta_{\mathrm{C}}$ 
(125 MHz, $\left.\mathrm{CDCl}_{3}\right) 13.2\left(\mathrm{CH}_{3}\right), 25.6\left(\mathrm{CH}_{2} \mathrm{CH}_{3}\right), 43.3(C(4)), 49.9(C(3)), 109.7(\mathrm{q}, J 3.5, C(5)), 118.5(\mathrm{q}, J$ 270, $\left.\mathrm{CF}_{3}\right), 121.6\left(\mathrm{C}(3) \mathrm{CH}=\mathrm{CHCH}_{2} \mathrm{CH}_{3}\right), 127.5(\mathrm{ArC}), 128.1(\mathrm{ArC}), 129.2(\mathrm{ArC}), 138.7$ (4ry $\left.\mathrm{C}(4) \mathrm{ArC}(1)\right)$, $138.8\left(\mathrm{C}(3) \mathrm{CH}=\mathrm{CHCH}_{2} \mathrm{CH}_{3}\right), 140.8(\mathrm{q}, J 37.9, C(6)), 166.2(C(2)) ; \delta_{\mathrm{F}}\left(376 \mathrm{MHz}, \mathrm{CDCl}_{3}\right)-72.6\left(\mathrm{CF}_{3}\right)$; Selected data or minor diastereoisomer: $\delta_{\mathrm{H}}\left(500 \mathrm{MHz}, \mathrm{CDCl}_{3}\right) 3.63(1 \mathrm{H}, \mathrm{t}, J$ 7.7, C(3)H), 3.87-3.90 $(1 \mathrm{H}$, m, $\mathrm{C}(4) H), 5.10\left(1 \mathrm{H}, \mathrm{ddt}, J 15.5,8.5,1.6, \mathrm{C}(3) \mathrm{CH}=\mathrm{CHCH}_{2} \mathrm{CH}_{3}\right), 5.70(1 \mathrm{H}, \mathrm{dt}, J$ 15.4, 6.4, $\left.\mathrm{C}(3) \mathrm{CH}=\mathrm{CHCH}_{2} \mathrm{CH}_{3}\right), 6.23(1 \mathrm{H}, \mathrm{d}, J 5.7, \mathrm{C}(5) H), 7.11(2 \mathrm{H}, \mathrm{d}, J$ 8.0, $\operatorname{Ar}(2,6) H) ; \delta_{\mathrm{C}}\left(125 \mathrm{MHz}, \mathrm{CDCl}_{3}\right)$ $43.0(C(4)), 47.6(C(3)), 110.7(\mathrm{q}, J 3.5, C(5)), 120.5\left(\mathrm{C}(3) C \mathrm{H}=\mathrm{CHCH}_{2} \mathrm{CH}_{3}\right), 128.2(\operatorname{ArC}), 128.2(A r C)$, $129.0(\mathrm{ArC}), 138.9\left(\mathrm{C}(3) \mathrm{CH}=\mathrm{CHCH}_{2} \mathrm{CH}_{3}\right), 166.6(C(2)) ; \delta_{\mathrm{F}}\left(376 \mathrm{MHz}, \mathrm{CDCl}_{3}\right)-72.6\left(\mathrm{CF}_{3}\right) ; m / z\left(\mathrm{NSI}^{+}\right)$ $297\left([\mathrm{M}+\mathrm{H}]^{+}, 20 \%\right)$; HRMS $\left(\mathrm{NSI}^{+}\right) \mathrm{C}_{16} \mathrm{H}_{16} \mathrm{~F}_{3} \mathrm{O}_{2}^{+}\left([\mathrm{M}+\mathrm{H}]^{+}\right)$requires 297.1097; found $297.1101(+1.4$ ppm).

\section{(3S,4R)-4-phenyl-3-((E)-styryl)-6-(trifluoromethyl)-3,4-dihydro-2H-pyran-2-one 38}

Following general procedure A, (E)-4-phenylbut-3-enoic acid 16 (64.9 mg, $0.40 \mathrm{mmol}), i \mathrm{Pr}_{2} \mathrm{NEt}(104 \mu \mathrm{L}$, $0.60 \mathrm{mmol})$ and pivaloyl chloride $(74.0 \mu \mathrm{L}, 0.60 \mathrm{mmol})$ in $\mathrm{CH}_{2} \mathrm{Cl}_{2}(2 \mathrm{~mL}), \mathrm{HBTM}-2.1(2 S, 3 R)-23(6.16$ mg, $0.02 \mathrm{mmol}, 5 \mathrm{~mol} \%)$, (E)-1,1,1-trifluoro-4-phenyl-3-buten-2-one 57 (80.0 mg, $0.40 \mathrm{mmol})$ and $i \operatorname{Pr}_{2}$ NEt $(174 \mu \mathrm{L}, 1.0 \mathrm{mmol})$ for 5 minutes at $-78{ }^{\circ} \mathrm{C}$ gave crude lactone $(3 S, 4 R)-38(95: 5 \mathrm{dr})$. Chromatographic purification (eluent $\mathrm{Et}_{2} \mathrm{O}$ :petrol 7.5:92.5) gave lactone $(3 S, 4 R)-38(95: 5 \mathrm{dr})$ as a colourless oil (113 mg, 82\%); $[\alpha]_{D}^{20}-159.6\left(c\right.$ 0.5, $\left.\mathrm{CH}_{2} \mathrm{Cl}_{2}\right)$; Chiral HPLC Chiralpak IB (5\% IPA:hexane, flow rate $\left.1 \mathrm{~mL} \min ^{-1}, 211 \mathrm{~nm}, 30^{\circ} \mathrm{C}\right)$ major diastereoisomer: $\mathrm{t}_{\mathrm{R}}(3 R, 4 S): 12.3 \mathrm{~min}, \mathrm{t}_{\mathrm{R}}(3 S, 4 R): 13.9 \mathrm{~min}$, $60 \% e e$; minor diastereoisomer $\mathrm{t}_{\mathrm{R}}: 8.00 \mathrm{~min}, \mathrm{t}_{\mathrm{R}}: 10.6 \mathrm{~min}, 52 \% e e ; v_{\max }(\mathrm{ATR}) / \mathrm{cm}^{-1} 3063,3030(\mathrm{C}-\mathrm{H})$, $1782(\mathrm{C}=\mathrm{O}), 1699,1601$; Data for major diastereoisomer: $\delta_{\mathrm{H}}\left(500 \mathrm{MHz}, \mathrm{CDCl}_{3}\right) 3.65(1 \mathrm{H}, \mathrm{t}, J$ 7.6, $\mathrm{C}(3) H), 3.85-3.90(1 \mathrm{H}, \mathrm{m}, \mathrm{C}(4) H), 6.15-6.20(2 \mathrm{H}, \mathrm{m}, \mathrm{C}(5) H$ and $\mathrm{C}(3) \mathrm{CH}=\mathrm{CHPh}), 6.36(1 \mathrm{H}, \mathrm{d}, J$ 15.9, $\mathrm{C}(3) \mathrm{CH}=\mathrm{CHPh}), 7.21(2 \mathrm{H}, \mathrm{d}, J$ 7.5, $\mathrm{ArH}), 7.28-7.42(8 \mathrm{H}, \mathrm{m}, \mathrm{Ar} H) ; \delta_{\mathrm{C}}\left(125 \mathrm{MHz}, \mathrm{CDCl}_{3}\right) 43.3(C(4))$, $50.0(C(3)), 109.9(\mathrm{q}, J 3.3, C(5)), 118.5\left(\mathrm{q}, J 270, C \mathrm{~F}_{3}\right), 122.0(\mathrm{C}(3) C \mathrm{H}=\mathrm{CHPh}), 126.6(A r C), 127.5$ $(A r C), 128.3(A r C), 128.4(A r C), 128.7(\mathrm{ArC}), 129.4(\mathrm{ArC}), 135.7(\mathrm{C}(3) \mathrm{CH}=C \mathrm{HPh}), 135.8(4 \mathrm{ry} A r C)$, 
138.5 (4ry $A r C), 141.0(\mathrm{q}, J 38.0, C(6)), 165.6(C(2))$; $\delta_{\mathrm{F}}\left(376 \mathrm{MHz}, \mathrm{CDCl}_{3}\right)-72.6\left(\mathrm{CF}_{3}\right)$; Selected data or minor diastereoisomer: $\delta_{\mathrm{H}}\left(500 \mathrm{MHz}, \mathrm{CDCl}_{3}\right) 3.99(1 \mathrm{H}, \mathrm{t}, J$ 6.2, C(4)H), $5.84(1 \mathrm{H}, \mathrm{dd}, J$ 16.0, 8.6, $\mathrm{C}(3) \mathrm{CH} H \mathrm{CHPh}), 6.30(1 \mathrm{H}, \mathrm{d}, J 5.9, \mathrm{C}(5) H), 6.59(1 \mathrm{H}, \mathrm{d}, J 16.0, \mathrm{C}(3) \mathrm{CH}=\mathrm{CHPh}), 7.16(2 \mathrm{H}, \mathrm{d}, J 7.6$, $\mathrm{ArH}) ; \delta_{\mathrm{C}}\left(125 \mathrm{MHz}, \mathrm{CDCl}_{3}\right) 47.9(C(3)), 110.7(\mathrm{q}, J 3.4, C(5)), 121.4(\mathrm{C}(3) C \mathrm{H}=\mathrm{CHPh}), 128.2(\operatorname{ArC})$, $128.5(A r C), 129.3(A r C), 135.4(\mathrm{C}(3) \mathrm{CH}=C \mathrm{HPh}), 166.2(C(2)) ; \delta_{\mathrm{F}}\left(376 \mathrm{MHz}, \mathrm{CDCl}_{3}\right)-72.7\left(\mathrm{CF}_{3}\right) ; m / z$ $\left(\mathrm{NSI}^{+}\right) 345\left([\mathrm{M}+\mathrm{H}]^{+}, 15 \%\right)$; HRMS $\left(\mathrm{NSI}^{+}\right) \mathrm{C}_{20} \mathrm{H}_{16} \mathrm{~F}_{3} \mathrm{O}_{2}^{+}\left([\mathrm{M}+\mathrm{H}]^{+}\right)$requires 345.1097; found 345.1098 $(+0.3 \mathrm{ppm})$.

\section{(3S,4R)-4-(4-bromophenyl)-3-((E)-prop-1-en-1-yl)-6-(trifluoromethyl)-3,4-dihydro-2H-pyran-2-one}

39

Following general procedure A, (E)-pent-3-enoic acid $24(40.6 \mu \mathrm{L}, 0.40 \mathrm{mmol}), i \operatorname{Pr}_{2} \mathrm{NEt}(104 \mu \mathrm{L}, 0.60$ mmol) and pivaloyl chloride $(74.0 \mu \mathrm{L}, 0.60 \mathrm{mmol})$ in $\mathrm{CH}_{2} \mathrm{Cl}_{2}(2 \mathrm{~mL}), \mathrm{HBTM}-2.1(2 S, 3 R)-23$ (6.16 mg, $0.02 \mathrm{mmol}, 5 \mathrm{~mol} \%)$, (E)-1,1,1-trifluoro-4-(4-bromophenyl)-3-buten-2-one 58 (112 mg, $0.40 \mathrm{mmol})$ and $i \operatorname{Pr}_{2} \mathrm{NEt}(174 \mu \mathrm{L}, 1.0 \mathrm{mmol})$ for 5 minutes at $\mathrm{rt}$ gave crude lactone $(3 S, 4 R)-39 \quad(80: 20 \mathrm{dr})$. Chromatographic purification (eluent $\mathrm{Et}_{2} \mathrm{O}$ :petrol 3:97) gave lactone $(3 S, 4 R)-39(95: 5 \mathrm{dr})$ as a colourless oil (105 mg, 73\%); $[\alpha]_{D}^{20}-201.0\left(c 0.5, \mathrm{CH}_{2} \mathrm{Cl}_{2}\right)$; Chiral HPLC Chiralcel OD-H (1\% IPA:hexane, flow rate

$\left.1 \mathrm{~mL} \min ^{-1}, 211 \mathrm{~nm}, 30{ }^{\circ} \mathrm{C}\right)$ major diastereoisomer: $\mathrm{t}_{\mathrm{R}}(3 R, 4 S): 10.1 \mathrm{~min}, \mathrm{t}_{\mathrm{R}}(3 S, 4 R): 11.8 \mathrm{~min}, 92 \%$ ee; minor diastereoisomer $t_{R}: 9.3 \mathrm{~min}, t_{R}: 12.8 \mathrm{~min}, 90 \% e e ; v_{\max }(A T R) / \mathrm{cm}^{-1} 2987(\mathrm{C}-\mathrm{H}), 1786(\mathrm{C}=\mathrm{O}), 1753$, 1660; Data for major diastereoisomer: $\delta_{\mathrm{H}}\left(500 \mathrm{MHz}, \mathrm{CDCl}_{3}\right) 1.69\left(3 \mathrm{H}, \mathrm{t}, J, 6.4, \mathrm{CH}_{3}\right), 3.37(1 \mathrm{H}, \mathrm{t}, J 7.4$, $\mathrm{C}(3) H), 3.68-3.71(1 \mathrm{H}, \mathrm{m}, \mathrm{C}(4) H), 5.43\left(1 \mathrm{H}, \mathrm{ddd}, J 15.3,7.5,1.3, \mathrm{C}(3) \mathrm{CH} H \mathrm{CHCH}_{3}\right), 5.52(1 \mathrm{H}, \mathrm{dq}, J 15.4$, 6.3, $\left.\mathrm{C}(3) \mathrm{CH}=\mathrm{CHCH}_{3}\right), 6.03(1 \mathrm{H}, \mathrm{d}, J 4.4, \mathrm{C}(5) H), 7.02-7.04(2 \mathrm{H}, \mathrm{m}, \mathrm{C}(4) \mathrm{Ar}(3,5) H), 7.50-7.52(2 \mathrm{H}, \mathrm{m}$, $\mathrm{C}(4) \operatorname{Ar}(2,6) H) ; \delta_{\mathrm{C}}\left(125 \mathrm{MHz}, \mathrm{CDCl}_{3}\right) 18.1\left(C_{3}\right), 42.7(C(4)), 49.7(C(3)), 109.2(\mathrm{q}, J 3.5, C(5)), 118.4$ $\left(\mathrm{q}, \quad J \quad 270, C \mathrm{~F}_{3}\right), \quad 122.1 \quad(\mathrm{C}(4) A r C(4)), \quad 123.4 \quad\left(\mathrm{C}(3) C H=\mathrm{CHCH}_{3}\right), \quad 129.1 \quad(\mathrm{C}(4) \operatorname{ArC}(3,5)), \quad 132.3$ $(\mathrm{C}(4) \operatorname{ArC}(2,6)), 132.6\left(\mathrm{C}(3) \mathrm{CH}=\mathrm{CHCH}_{3}\right), 137.7$ (4ry C(4)ArC(1)), $141.1(\mathrm{q}, J 38, C(6)), 165.8(C(2)) ; \delta_{\mathrm{F}}$ $\left(376 \mathrm{MHz}, \mathrm{CDCl}_{3}\right)-72.7\left(\mathrm{CF}_{3}\right)$; Selected data or minor diastereoisomer: $\delta_{\mathrm{H}}\left(500 \mathrm{MHz}, \mathrm{CDCl}_{3}\right) 3.62(1 \mathrm{H}$, 
$\mathrm{t}, J$ 7.8, C(3)H), 3.82-3.85 $(1 \mathrm{H}, \mathrm{m}, \mathrm{C}(4) H), 5.09-5.14\left(1 \mathrm{H}, \mathrm{m}, \mathrm{C}(3) \mathrm{CH}=\mathrm{CHCH}_{3}\right), 5.65-5.72(1 \mathrm{H}, \mathrm{m}$, $\left.\mathrm{C}(3) \mathrm{CH}=\mathrm{CHCH}_{3}\right), 6.18(1 \mathrm{H}, \mathrm{d}, J 5.7, \mathrm{C}(5) H) ; \delta_{\mathrm{C}}\left(75 \mathrm{MHz}, \mathrm{CDCl}_{3}\right) 18.0\left(C_{3}\right), 44.4(C(4)), 47.4(C(3))$; $\delta_{\mathrm{F}}\left(376 \mathrm{MHz}, \mathrm{CDCl}_{3}\right)-72.8\left(\mathrm{CF}_{3}\right) ; m / z\left(\mathrm{NSI}^{+}\right) 378\left(\left[\mathrm{M}+\mathrm{NH}_{4}\right]^{+}, 56 \%\right) ; \mathrm{HRMS}\left(\mathrm{NSI}^{+}\right) \mathrm{C}_{15} \mathrm{H}_{16}{ }^{78} \mathrm{BrF}_{3} \mathrm{NO}_{2}^{+}$ $\left(\left[\mathrm{M}+\mathrm{NH}_{4}\right]^{+}\right)$requires 378.0311 ; found $378.0311(+0.0 \mathrm{ppm})$.

\section{(3S,4R)-4-(thiophen-2-yl)-3-((E)-prop-1-en-1-yl)-6-(trifluoromethyl)-3,4-dihydro-2H-pyran-2-one 40}

Following general procedure A, (E)-pent-3-enoic acid $24(40.6 \mu \mathrm{L}, 0.40 \mathrm{mmol}), i \mathrm{Pr}_{2} \mathrm{NEt}(104 \mu \mathrm{L}, 0.60$ mmol) and pivaloyl chloride $(74.0 \mu \mathrm{L}, 0.60 \mathrm{mmol})$ in $\mathrm{CH}_{2} \mathrm{Cl}_{2}(2 \mathrm{~mL}), \mathrm{HBTM}-2.1(2 S, 3 R)-23(6.16 \mathrm{mg}$, $0.02 \mathrm{mmol}, 5 \mathrm{~mol} \%$ ), (E)-1,1,1-trifluoro-4-(2-thienyl)-3-buten-2-one 59 (82.4 mg, $0.40 \mathrm{mmol})$ and $i \operatorname{Pr}_{2} \mathrm{NEt}(174 \mu \mathrm{L}, 1.0 \mathrm{mmol})$ for 5 minutes at $\mathrm{rt}$ gave crude lactone $(3 S, 4 R)-40 \quad(84: 16 \mathrm{dr})$. Chromatographic purification (eluent $\mathrm{Et}_{2} \mathrm{O}$ :petrol 4:96) gave lactone $(3 S, 4 R)-\mathbf{4 0}(84: 16 \mathrm{dr})$ as a colourless oil (95.8 mg, 83\%); $[\alpha]_{D}^{20}-187.0\left(c 0.5, \mathrm{CH}_{2} \mathrm{Cl}_{2}\right)$; Chiral HPLC Chiralpak AS-H (0.5\% IPA:hexane, flow rate $\left.1 \mathrm{~mL} \mathrm{~min}^{-1}, 211 \mathrm{~nm}, 30{ }^{\circ} \mathrm{C}\right)$ major diastereoisomer: $\mathrm{t}_{\mathrm{R}}(3 S, 4 R): 14.1 \mathrm{~min}, \mathrm{t}_{\mathrm{R}}(3 R, 4 S): 16.1 \mathrm{~min}, 93 \%$ ee; $v_{\max }(\mathrm{ATR}) / \mathrm{cm}^{-1} 2998(\mathrm{C}-\mathrm{H}), 1784(\mathrm{C}=\mathrm{O}), 1699,1674$; Data for major diastereoisomer: $\delta_{\mathrm{H}}(300 \mathrm{MHz}$, $\left.\mathrm{CDCl}_{3}\right)$ 1.72-1.74 (3H, m, $\left.\mathrm{CH}_{3}\right), 3.55(1 \mathrm{H}, \mathrm{td}, J$ 6.7, 0.7, C(3)H), 4.00-4.04 (1H, m, C(4)H), $5.47(1 \mathrm{H}$, ddq, $\left.J 15.4,8.4,1.7, \mathrm{C}(3) \mathrm{CH}=\mathrm{CHCH}_{3}\right), 5.63-5.75\left(1 \mathrm{H}, \mathrm{m}, \mathrm{C}(3) \mathrm{CH}=\mathrm{CHCH}_{3}\right), 6.16(1 \mathrm{H}, \mathrm{d}, J 5.0, \mathrm{C}(5) H)$, 6.89-6.90 (1H, m, ArH), 6.98-7.03 (1H, m, ArH), 7.27-7.30 (1H, m, ArH); $\delta_{\mathrm{C}}\left(125 \mathrm{MHz}, \mathrm{CDCl}_{3}\right) 18.1$ $\left(C \mathrm{H}_{3}\right), 38.2(C(4)), 50.6(C(3)), 109.2(\mathrm{q}, J 3.5, C(5)), 118.4\left(\mathrm{q}, J 270, C \mathrm{~F}_{3}\right), 123.2\left(\mathrm{C}(3) C \mathrm{H}=\mathrm{CHCH}_{3}\right)$, $125.4(A r C), 125.4(A r C), 127.4(A r C), 132.4\left(\mathrm{C}(3) \mathrm{CH}=\mathrm{CHCH}_{3}\right), 140.7$ (q, $J$ 37.9, $\left.C(6)\right), 141.2$ $(\mathrm{C}(4) \operatorname{ArC}(1)), 165.5(C(2)) ; \delta_{\mathrm{F}}\left(376 \mathrm{MHz}, \mathrm{CDCl}_{3}\right)-72.4\left(\mathrm{CF}_{3}\right)$; Selected data or minor diastereoisomer: $\delta_{\mathrm{H}}$ $\left(500 \mathrm{MHz}, \mathrm{CDCl}_{3}\right) 3.64(1 \mathrm{H}, \mathrm{t}, J$ 7.5, C(3)H), 4.13-4.18 (1H, m, C(4)H), $5.35(1 \mathrm{H}, \mathrm{ddq}, J$ 15.4, 8.4, 1.7, $\left.\mathrm{C}(3) \mathrm{CH}=\mathrm{CHCH}_{3}\right), 6.28(1 \mathrm{H}, \mathrm{d}, J 5.8, \mathrm{C}(5) H) ; \delta_{\mathrm{C}}\left(75 \mathrm{MHz}, \mathrm{CDCl}_{3}\right) 18.1\left(\mathrm{CH}_{3}\right), 38.0(C(4)), 48.0(C(3))$, $110.7(\mathrm{q}, \quad J$ 3.5, $C(5)), 122.5\left(\mathrm{C}(3) C \mathrm{H}=\mathrm{CHCH}_{3}\right), 125.7(\operatorname{ArC}), 126.3(\operatorname{ArC}), 127.4(\operatorname{ArC}), 132.7$ $\left(\mathrm{C}(3) \mathrm{CH}=\mathrm{CHCH}_{3}\right), 166.2(C(2)) ; \delta_{\mathrm{F}}\left(376 \mathrm{MHz}, \mathrm{CDCl}_{3}\right)-72.6\left(\mathrm{CF}_{3}\right) ; m / z\left(\mathrm{APCI}^{+}\right) 289\left([\mathrm{M}+\mathrm{H}]^{+}, 100 \%\right) ;$ HRMS $\left(\mathrm{APCI}^{+}\right) \mathrm{C}_{13} \mathrm{H}_{12} \mathrm{~F}_{3} \mathrm{O}_{2} \mathrm{~S}^{+}\left([\mathrm{M}+\mathrm{H}]^{+}\right)$requires 289.0505; found 289.0507 (+0.8 ppm). 
Following general procedure A, (E)-pent-3-enoic acid $24(40.6 \mu \mathrm{L}, 0.40 \mathrm{mmol}), i \mathrm{Pr}_{2} \mathrm{NEt}(104 \mu \mathrm{L}, 0.60$ mmol) and pivaloyl chloride $(74.0 \mu \mathrm{L}, 0.60 \mathrm{mmol})$ in $\mathrm{CH}_{2} \mathrm{Cl}_{2}(2 \mathrm{~mL}), \mathrm{HBTM}-2.1(2 S, 3 R)-23(1.23 \mathrm{mg}$, $0.004 \mathrm{mmol}, 1 \mathrm{~mol} \%),(\mathrm{NE})-\mathrm{N}-\left(\right.$ phenylimino)benzamide $60(84.0 \mathrm{mg}, 0.40 \mathrm{mmol})$ and $i \operatorname{Pr}_{2} \mathrm{NEt}(174 \mu \mathrm{L}$, $1.00 \mathrm{mmol}$ ) for $15 \mathrm{~min}$ at $\mathrm{rt}$, followed by addition of $\mathrm{MeOH}(2 \mathrm{~mL})$ and stirring for $1 \mathrm{~h}$ at $\mathrm{rt}$ gave, after chromatographic purification (eluent $\mathrm{Et}_{2} \mathrm{O}$ :petrol 40:60) a rotameric mixture (ratio 95:5) of (2R)-41 as a white solid (105 mg, 80\%); mp 108-110 ${ }^{\circ} \mathrm{C} ;[\alpha]_{D}^{20}-67.0\left(c\right.$ 0.5, $\left.\mathrm{CH}_{2} \mathrm{Cl}_{2}\right)$; Chiral HPLC Chiralpak IB (5\% IPA:hexane, flow rate $\left.1 \mathrm{~mL} \min ^{-1}, 211 \mathrm{~nm}, 30{ }^{\circ} \mathrm{C}\right) \mathrm{t}_{\mathrm{R}}(2 S): 13.2 \mathrm{~min}, \mathrm{t}_{\mathrm{R}}(2 R): 17.3 \mathrm{~min}, 96 \%$ ee; $v_{\max }$ $(\mathrm{ATR}) / \mathrm{cm}^{-1} 3350(\mathrm{~N}-\mathrm{H}), 2949(\mathrm{C}-\mathrm{H}), 1721(\mathrm{C}=\mathrm{O}), 1698(\mathrm{C}=\mathrm{O}), 1597$; Data for major rotamer: $\delta_{\mathrm{H}}(500$ $\left.\mathrm{MHz}, \mathrm{CDCl}_{3}\right) 1.72\left(3 \mathrm{H}, \mathrm{d}, J\right.$ 4.7, $\left.\mathrm{CH}_{3} \mathrm{CH}=\right), 3.78\left(3 \mathrm{H}, \mathrm{s}, \mathrm{OCH}_{3}\right), 5.25-5.26(1 \mathrm{H}, \mathrm{m}, \mathrm{C}(2) H), 5.79-5.89$ $\left(2 \mathrm{H}, \mathrm{m}, \mathrm{CH}=\mathrm{CHCH}_{3}\right.$ and $\left.\mathrm{CH}=\mathrm{CHCH}_{3}\right), 6.94-6.97(3 \mathrm{H}, \mathrm{m}, \mathrm{NAr}(2,6) H$ and $\mathrm{NAr}(4) H), 7.27-7.30(2 \mathrm{H}, \mathrm{m}$, $\mathrm{NAr}(3,5) H), 7.50(2 \mathrm{H}, \mathrm{t}, J$ 7.6, C(O)Ar(3,5)H), $7.58(1 \mathrm{H}, \mathrm{t}, J$ 7.4, C(O)Ar(4)H), 7.86-7.88 (2H, m, $\mathrm{C}(\mathrm{O}) \operatorname{Ar}(2,6) H), 8.64(1 \mathrm{H}, \mathrm{s}, \mathrm{NH}) ; \delta_{\mathrm{C}}\left(125 \mathrm{MHz}, \mathrm{CDCl}_{3}\right) 18.2\left(\mathrm{CH}_{3} \mathrm{CH}=\right), 52.4\left(\mathrm{OCH}_{3}\right), 64.6(C(2)), 114.7$ $(\mathrm{N} A r C(2,6)), 121.5(\mathrm{NArC}(4)), 123.6\left(C \mathrm{H}=\mathrm{CHCH}_{3}\right), 127.2(\mathrm{C}(\mathrm{O}) \operatorname{ArC}(2,6)), 128.8(\mathrm{C}(\mathrm{O}) \operatorname{ArC}(3,5)), 129.4$ $(\mathrm{NArC}(3,5)), 132.0\left(\mathrm{C}(\mathrm{O}) \operatorname{ArC}(4)\right.$ or $\left.\mathrm{CH}=\mathrm{CHCH}_{3}\right), 132.3(\mathrm{C}(\mathrm{O}) \operatorname{ArC}(4))$ or $\mathrm{CH}=\mathrm{CHCH}$ ), 133.0 (4ry $\mathrm{C}(\mathrm{O}) \operatorname{ArC}(1)), 148.1(\mathrm{~N} A r C(1)), 167.4(\mathrm{NHC}=\mathrm{O}), 173.3(\mathrm{MeOC}=\mathrm{O})$; Selected data for minor rotamer: $\delta_{\mathrm{H}}$ $\left(500 \mathrm{MHz}, \mathrm{CDCl}_{3}\right) 1.65\left(3 \mathrm{H}, \mathrm{d}, J 5.9, \mathrm{CH}_{3} \mathrm{CH}=\right), 3.67\left(3 \mathrm{H}, \mathrm{s}, \mathrm{OCH}_{3}\right), 4.97(1 \mathrm{H}, \mathrm{d}, J 7.1, \mathrm{C}(2) H) ; \delta_{\mathrm{C}}(125$ $\left.\mathrm{MHz}, \mathrm{CDCl}_{3}\right) 18.1\left(\mathrm{CH}_{3} \mathrm{CH}=\right), 52.3\left(\mathrm{OCH}_{3}\right), 65.8(C(2)), 115.0(\mathrm{~N} A r C(2,6)) ; m / z\left(\mathrm{NSI}^{+}\right) 325\left([\mathrm{M}+\mathrm{H}]^{+}\right.$, $100 \%)$; HRMS $\left(\mathrm{NSI}^{+}\right) \mathrm{C}_{19} \mathrm{H}_{21} \mathrm{~N}_{2} \mathrm{O}_{3}{ }^{+}\left([\mathrm{M}+\mathrm{H}]^{+}\right)$requires 325.1547 ; found $325.1548(+0.4 \mathrm{ppm})$.

\section{(2R)-(E)-methyl 2-(2-benzoyl-1-phenylhydrazinyl)hex-3-enoate 42}

Following general procedure A, (E)-hex-3-enoic acid $81(47.4 \mu \mathrm{L}, 0.40 \mathrm{mmol}), i \mathrm{Pr}_{2} \mathrm{NEt}(104 \mu \mathrm{L}, 0.60$ mmol) and pivaloyl chloride $(74.0 \mu \mathrm{L}, 0.60 \mathrm{mmol})$ in $\mathrm{CH}_{2} \mathrm{Cl}_{2}(2 \mathrm{~mL}), \mathrm{HBTM}-2.1(2 S, 3 R)-23(1.23 \mathrm{mg}$, $0.004 \mathrm{mmol}, 1 \mathrm{~mol} \%),(\mathrm{NE})-\mathrm{N}-\left(\right.$ phenylimino)benzamide $60(84.0 \mathrm{mg}, 0.40 \mathrm{mmol})$ and $i \operatorname{Pr}_{2} \mathrm{NEt}(174 \mu \mathrm{L}$, $1.00 \mathrm{mmol})$ for $15 \mathrm{~min}$ at $\mathrm{rt}$, followed by addition of $\mathrm{MeOH}(2 \mathrm{~mL})$ and stirring for $1 \mathrm{~h}$ at $\mathrm{rt}$ gave, after 
chromatographic purification (eluent $\mathrm{Et}_{2} \mathrm{O}$ :petrol 40:60) a rotameric mixture (ratio 95:5) of (2R)-42 as a white solid (115 mg, 85\%); mp 98-100 ${ }^{\circ} \mathrm{C} ;[\alpha]_{D}^{20}-54.8\left(c \quad 0.5, \mathrm{CH}_{2} \mathrm{Cl}_{2}\right)$; Chiral HPLC Chiralpak IB $(5 \%$

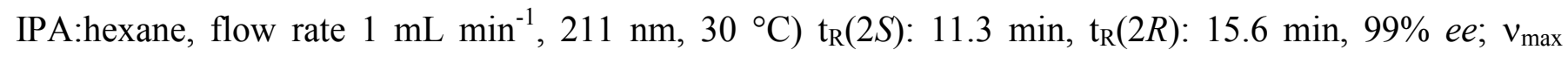
$(\mathrm{ATR}) / \mathrm{cm}^{-1} 3352(\mathrm{~N}-\mathrm{H}), 2990(\mathrm{C}-\mathrm{H}), 1721(\mathrm{C}=\mathrm{O}), 1688(\mathrm{C}=\mathrm{O}), 1597,1508$; Data for major rotamer: $\delta_{\mathrm{H}}$ $\left(500 \mathrm{MHz}, \mathrm{CDCl}_{3}\right) 0.90\left(3 \mathrm{H}, \mathrm{t}, J\right.$ 7.4, $\left.\mathrm{CH}_{3} \mathrm{CH}_{2}\right), 2.03-2.09\left(2 \mathrm{H}, \mathrm{m}, \mathrm{CH}_{3} \mathrm{CH}_{2}\right) 3.78\left(3 \mathrm{H}, \mathrm{s}, \mathrm{OCH}_{3}\right), 5.26-$ $5.27(1 \mathrm{H}, \mathrm{m}, \mathrm{C}(2) H), 5.77-5.88\left(2 \mathrm{H}, \mathrm{m}, \mathrm{CH}=\mathrm{CHCH}_{2} \mathrm{CH}_{3}\right.$ and $\left.\mathrm{CH}=\mathrm{CHCH}_{2} \mathrm{CH}_{3}\right)$, 6.93-6.97 (3H, m, $\mathrm{NAr}(2,6) H$ and $\mathrm{NAr}(4) H), 7.26-7.29(2 \mathrm{H}, \mathrm{m}, \mathrm{NAr}(3,5) H), 7.47-7.50(2 \mathrm{H}, \mathrm{m}, \mathrm{C}(\mathrm{O}) \operatorname{Ar}(3,5) H), 7.55-7.58$ $(1 \mathrm{H}, \mathrm{m}, \mathrm{C}(\mathrm{O}) \operatorname{Ar}(4) H), 7.85-7.87(2 \mathrm{H}, \mathrm{m}, \mathrm{C}(\mathrm{O}) \operatorname{Ar}(2,6) H), 8.62(1 \mathrm{H}, \mathrm{s}, \mathrm{NH}) ; \delta_{\mathrm{C}}\left(125 \mathrm{MHz}, \mathrm{CDCl}_{3}\right) 13.1$ $\left(\mathrm{CH}_{3} \mathrm{CH}_{2}\right), 25.6 \quad\left(\mathrm{CH}_{3} \mathrm{CH}_{2}\right), \quad 52.4 \quad\left(\mathrm{OCH}_{3}\right), 64.5 \quad(C(2)), 114.7 \quad(\mathrm{~N} A r C(2,6)), 121.6 \quad(\mathrm{~N} A r C(4)$ or $\left.C \mathrm{H}=\mathrm{CHCH}_{2} \mathrm{CH}_{3}\right), 121.7\left(\mathrm{NArC}(4)\right.$ or $\left.\mathrm{CH}=\mathrm{CHCH}_{2} \mathrm{CH}_{3}\right), 127.3(\mathrm{C}(\mathrm{O}) A r C(2,6)), 128.9(\mathrm{C}(\mathrm{O}) A r C(3,5))$, $129.4(\mathrm{~N} A r C(3,5)), 132.1(\mathrm{C}(\mathrm{O}) \operatorname{ArC}(4)), 133.1(\mathrm{C}(\mathrm{O}) \operatorname{ArC}(1)), 138.8\left(\mathrm{CH}=\mathrm{CHCH}_{2} \mathrm{CH}_{3}\right), 148.2(\mathrm{~N} A r C(1))$, $167.4(\mathrm{NHC}=\mathrm{O}), 173.4(\mathrm{MeOC}=\mathrm{O})$; Selected data for minor rotamer: $\delta_{\mathrm{H}}\left(500 \mathrm{MHz}, \mathrm{CDCl}_{3}\right) 3.67(3 \mathrm{H}, \mathrm{s}$, $\left.\mathrm{OCH}_{3}\right), 4.99-5.00(1 \mathrm{H}, \mathrm{m}, \mathrm{C}(2) H) ; \delta_{\mathrm{C}}\left(125 \mathrm{MHz}, \mathrm{CDCl}_{3}\right) 12.5\left(\mathrm{CH}_{3} \mathrm{CH}_{2}\right), 52.3\left(\mathrm{OCH}_{3}\right), 66.1(C(2)), 115.1$ $(\mathrm{N} A r C(2,6)), 141.1\left(\mathrm{CH}=C \mathrm{HCH}_{2} \mathrm{CH}_{3}\right), 148.7(\mathrm{~N} A r C(1)), 167.4(\mathrm{NHC}=\mathrm{O}), 173.4(\mathrm{MeOC}=\mathrm{O}) ; m / z\left(\mathrm{NSI}^{+}\right)$ $339\left([\mathrm{M}+\mathrm{H}]^{+}, 100 \%\right)$; HRMS $\left(\mathrm{NSI}^{+}\right) \mathrm{C}_{20} \mathrm{H}_{23} \mathrm{~N}_{2} \mathrm{O}_{3}{ }^{+}\left([\mathrm{M}+\mathrm{H}]^{+}\right)$requires 339.1703; found $339.1708(+1.4$ ppm).

\section{(2R)-(E)-methyl 2-(2-benzoyl-1-phenylhydrazinyl)-5-methylhex-3-enoate 43}

Following general procedure A, (E)-5-methylhex-3-enoic acid 65 (51.2 mg, $0.40 \mathrm{mmol}), i \operatorname{Pr}_{2} \mathrm{NEt}(104 \mu \mathrm{L}$, $0.60 \mathrm{mmol})$ and pivaloyl chloride $(74.0 \mu \mathrm{L}, 0.60 \mathrm{mmol})$ in $\mathrm{CH}_{2} \mathrm{Cl}_{2}(2 \mathrm{~mL}), \mathrm{HBTM}-2.1(2 S, 3 R)-23(1.23$ mg, 0.004 mmol, 1 mol\%), (NE)-N-(phenylimino)benzamide 60 (84.0 mg, $0.40 \mathrm{mmol})$ and $i \operatorname{Pr}_{2} \mathrm{NEt}(174$ $\mu \mathrm{L}, 1.00 \mathrm{mmol})$ for $15 \mathrm{~min}$ at $\mathrm{rt}$, followed by addition of $\mathrm{MeOH}(2 \mathrm{~mL})$ and stirring for $1 \mathrm{~h}$ at $\mathrm{rt}$ gave, after chromatographic purification (eluent $\mathrm{Et}_{2} \mathrm{O}$ :petrol 40:60) a rotameric mixture (ratio 95:5) of (2R)-43 as a white solid (104 mg, 74\%); mp 128-130 ${ }^{\circ} \mathrm{C} ;[\alpha]_{D}^{20}-66.6\left(c 0.5, \mathrm{CH}_{2} \mathrm{Cl}_{2}\right)$; Chiral HPLC Chiralpak IB $(5 \%$ IPA:hexane, flow rate $\left.1 \mathrm{~mL} \mathrm{~min}^{-1}, 211 \mathrm{~nm}, 30{ }^{\circ} \mathrm{C}\right) \mathrm{t}_{\mathrm{R}}(2 S): 14.5 \mathrm{~min}, \mathrm{t}_{\mathrm{R}}(2 R): 19.0 \mathrm{~min}, 99 \%$ ee; $v_{\max }$ 
$(\mathrm{ATR}) / \mathrm{cm}^{-1} 3326(\mathrm{~N}-\mathrm{H}), 2988(\mathrm{C}-\mathrm{H}), 1719(\mathrm{C}=\mathrm{O}$ Ester $), 1678$ (C=O Amide), 1597, 1506; Data for major rotamer: $\delta_{\mathrm{H}}\left(500 \mathrm{MHz}, \mathrm{CDCl}_{3}\right) 0.89\left(3 \mathrm{H}, \mathrm{t}, J 6.8, \mathrm{CH}\left(\mathrm{CH}_{3}\right) \mathrm{CH}_{3}\right), 0.92\left(3 \mathrm{H}, \mathrm{t}, J\right.$ 6.8, $\left.\mathrm{CH}\left(\mathrm{CH}_{3}\right) \mathrm{CH}_{3}\right), 2.27-$ $2.34\left(1 \mathrm{H}, \mathrm{m}, \mathrm{CH}\left(\mathrm{CH}_{3}\right) \mathrm{CH}_{3}\right), 3.78\left(3 \mathrm{H}, \mathrm{s}, \mathrm{OCH}_{3}\right), 5.27-5.28(1 \mathrm{H}, \mathrm{m}, \mathrm{C}(2) H)$, 5.72-5.80 (2H, m, $\mathrm{CH}=\mathrm{CHCH}\left(\mathrm{CH}_{3}\right) \mathrm{CH}_{3}$ and $\left.\mathrm{CH}=\mathrm{CHCH}\left(\mathrm{CH}_{3}\right) \mathrm{CH}_{3}\right), 6.93-6.97(3 \mathrm{H}, \mathrm{m}, \mathrm{NAr}(2,6) H$ and $\mathrm{NAr}(4) H), 7.26-7.30$ $(2 \mathrm{H}, \mathrm{m}, \mathrm{NAr}(3,5) H), 7.46-7.49(2 \mathrm{H}, \mathrm{m}, \mathrm{C}(\mathrm{O}) \operatorname{Ar}(3,5) H), 7.54-7.58(1 \mathrm{H}, \mathrm{m}, \mathrm{C}(\mathrm{O}) \operatorname{Ar}(4) H), 7.85-7.88(2 \mathrm{H}$, m, C(O)Ar(2,6)H), $8.64(1 \mathrm{H}, \mathrm{s}, \mathrm{NH}) ; \delta_{\mathrm{C}}\left(100 \mathrm{MHz}, \mathrm{CDCl}_{3}\right) 21.8\left(\mathrm{CH}\left(\mathrm{CH}_{3}\right) \mathrm{CH}_{3}\right), 22.0\left(\mathrm{CH}\left(\mathrm{CH}_{3}\right) \mathrm{CH}_{3}\right)$, $31.1\left(\mathrm{CH}\left(\mathrm{CH}_{3}\right) \mathrm{CH}_{3}\right), 52.3\left(\mathrm{OCH}_{3}\right), 64.4(\mathrm{C}(2)), 114.8(\mathrm{NArC}(2,6)), 120.0\left(\mathrm{CH}=\mathrm{CHCH}\left(\mathrm{CH}_{3}\right) \mathrm{CH}_{3}\right), 121.5$ (NArC(4)), $127.3(\mathrm{C}(\mathrm{O}) \operatorname{ArC}(2,6)), 128.8(\mathrm{C}(\mathrm{O}) \operatorname{ArC}(3,5)), 129.4(\mathrm{~N} A r C(3,5)), 132.1(\mathrm{C}(\mathrm{O}) \operatorname{ArC}(4)), 133.0$ $(\mathrm{C}(\mathrm{O}) \operatorname{ArC}(1)), 143.9\left(\mathrm{CH}=C \mathrm{HCH}\left(\mathrm{CH}_{3}\right) \mathrm{CH}_{3}\right), 148.2(\mathrm{NArC}(1)), 167.3(\mathrm{NHC}=\mathrm{O}), 173.4(\mathrm{MeOC}=\mathrm{O})$; Selected data for minor rotamer: $\delta_{\mathrm{H}}\left(500 \mathrm{MHz}, \mathrm{CDCl}_{3}\right) 3.66\left(3 \mathrm{H}, \mathrm{s}, \mathrm{OCH}_{3}\right), 5.01(1 \mathrm{H}, \mathrm{d}, J 7.0, \mathrm{C}(2) H) ; \delta_{\mathrm{C}}$

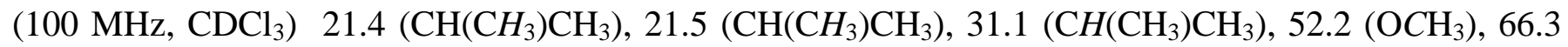
$(C(2)), 115.2(\mathrm{NArC}(2,6)), 146.1\left(\mathrm{CH}=C \mathrm{HCH}_{2} \mathrm{CH}_{3}\right), 148.8(\mathrm{NArC}(1)), 172.0(\mathrm{MeOC}=\mathrm{O}) ; m / z\left(\mathrm{NSI}^{+}\right) 353$ $\left([\mathrm{M}+\mathrm{H}]^{+}, 100 \%\right) ; \mathrm{HRMS}\left(\mathrm{NSI}^{+}\right) \mathrm{C}_{21} \mathrm{H}_{25} \mathrm{~N}_{2} \mathrm{O}_{3}{ }^{+}\left([\mathrm{M}+\mathrm{H}]^{+}\right)$requires 353.1860; found $353.1862(+0.7 \mathrm{ppm})$

\section{(2R)-(E)-methyl 2-(2-benzoyl-1-phenylhydrazinyl)-5-phenylpent-3-enoate 44}

Following general procedure A, (E)-5-phenylpent-3-enoic acid $66(70.4 \mathrm{mg}, 0.40 \mathrm{mmol}), i \mathrm{Pr}_{2} \mathrm{NEt}(104 \mu \mathrm{L}$, $0.60 \mathrm{mmol})$ and pivaloyl chloride $(74.0 \mu \mathrm{L}, 0.60 \mathrm{mmol})$ in $\mathrm{CH}_{2} \mathrm{Cl}_{2}(2 \mathrm{~mL}), \mathrm{HBTM}-2.1(2 S, 3 R)-23(1.23$ mg, 0.004 mmol, 1 mol\%), (NE)-N-(phenylimino)benzamide 60 (84.0 mg, $0.40 \mathrm{mmol})$ and $i \operatorname{Pr}_{2} \mathrm{NEt}(174$ $\mu \mathrm{L}, 1.00 \mathrm{mmol}$ ) for $15 \mathrm{~min}$ at $\mathrm{rt}$, followed by addition of $\mathrm{MeOH}(2 \mathrm{~mL})$ and stirring for $1 \mathrm{~h}$ at $\mathrm{rt}$ gave, after chromatographic purification (eluent $\mathrm{Et}_{2} \mathrm{O}$ :petrol 50:50) a rotameric mixture (ratio 96:4) of (2R)-44 as a white solid (123 mg, 77\%); mp 136-138 ${ }^{\circ} \mathrm{C} ;[\alpha]_{D}^{20}-70.8\left(c 0.5, \mathrm{CH}_{2} \mathrm{Cl}_{2}\right)$; Chiral HPLC Chiralpak IB $(5 \%$

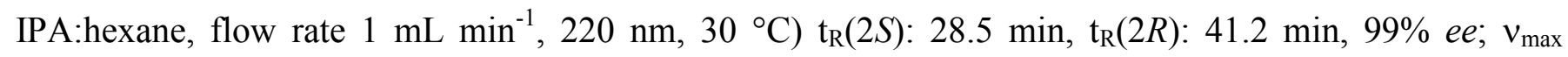
$(\mathrm{ATR}) / \mathrm{cm}^{-1} 3323(\mathrm{~N}-\mathrm{H}), 2890(\mathrm{C}-\mathrm{H}), 1730(\mathrm{C}=\mathrm{O}$ Ester $), 1686(\mathrm{C}=\mathrm{O}$ Amide $), 1599$, 1514; Data for major rotamer: $\delta_{\mathrm{H}}\left(500 \mathrm{MHz}, \mathrm{CDCl}_{3}\right) 3.41(2 \mathrm{H}, \mathrm{d}, J$ 6.1, CHHPh and $\mathrm{CH} H \mathrm{Ph}), 3.78\left(3 \mathrm{H}, \mathrm{s}, \mathrm{OCH}_{3}\right), 5.34-5.35$ $(1 \mathrm{H}, \mathrm{m}, \mathrm{C}(2) H), 5.89-6.00(2 \mathrm{H}, \mathrm{m}, \mathrm{CH}=\mathrm{CHBn}$ and $\mathrm{CH}=\mathrm{CHBn}), 6.96-6.99(3 \mathrm{H}, \mathrm{m}, \mathrm{NAr}(2,6) H$ and $\mathrm{NAr}(4) H), 7.05-7.03\left(\mathrm{CH}_{2} \mathrm{Ar}(2,6) H\right), 7.12-7.15\left(\mathrm{CH}_{2} \mathrm{Ar}(3,5) H\right.$ and $\left.\mathrm{CH}_{2} \mathrm{Ar}(4) H\right), 7.28-7.32(2 \mathrm{H}, \mathrm{m}$, 
$\mathrm{NAr}(3,5) H), 7.48-7.51(2 \mathrm{H}, \mathrm{m}, \mathrm{C}(\mathrm{O}) \operatorname{Ar}(3,5) H), 7.58-7.62(1 \mathrm{H}, \mathrm{m}, \mathrm{C}(\mathrm{O}) \operatorname{Ar}(4) H), 7.85-7.86(2 \mathrm{H}, \mathrm{m}$, $\mathrm{C}(\mathrm{O}) \operatorname{Ar}(2,6) H), 8.68(1 \mathrm{H}, \mathrm{s}, \mathrm{NH}) ; \delta_{\mathrm{C}}\left(100 \mathrm{MHz}, \mathrm{CDCl}_{3}\right) 38.9\left(\mathrm{CH}_{2} \mathrm{Ph}\right), 52.4\left(\mathrm{OCH}_{3}\right), 64.5(C(2)), 114.7$ (NArC(2,6)), $121.6(\mathrm{NArC}(4)), 124.1(\mathrm{CH}=\mathrm{CHBn}), 126.2\left(\mathrm{CH}_{2} \operatorname{ArC}(4)\right), 127.3(\mathrm{C}(\mathrm{O}) \operatorname{ArC}(2,6)), 128.4$ (ArC), $128.6(\operatorname{ArC}), 128.8(\mathrm{C}(\mathrm{O}) \operatorname{ArC}(3,5)), 129.4(\mathrm{~N} A r C(3,5)), 132.1(\mathrm{C}(\mathrm{O}) \operatorname{ArC}(4)), 132.8(\mathrm{C}(\mathrm{O}) \operatorname{ArC}(1))$, $135.7(\mathrm{CH}=C H B n), 138.9\left(\mathrm{CH}_{2} A r C(1)\right), 148.1(\mathrm{~N} A r C(1)), 167.1(\mathrm{NHC}=\mathrm{O}), 173.1(\mathrm{MeOC}=\mathrm{O})$; Selected data for minor rotamer $\delta_{\mathrm{H}}\left(500 \mathrm{MHz}, \mathrm{CDCl}_{3}\right) 3.67\left(3 \mathrm{H}, \mathrm{s}, \mathrm{OCH}_{3}\right), 5.06-5.08(1 \mathrm{H}, \mathrm{m}, \mathrm{C}(2) H) ; \delta_{\mathrm{C}}(100$ $\left.\mathrm{MHz}, \mathrm{CDCl}_{3}\right) 38.8\left(\mathrm{CH}_{2} \mathrm{Ph}\right), 52.3\left(\mathrm{OCH}_{3}\right), 66.2(C(2)), 115.2(\mathrm{~N} A r C(2,6)), 148.7(\mathrm{~N} A r C(1)) ; m / z\left(\mathrm{NSI}^{+}\right)$ $401\left([\mathrm{M}+\mathrm{H}]^{+}, 100 \%\right) ; \mathrm{HRMS}\left(\mathrm{NSI}^{+}\right) \mathrm{C}_{25} \mathrm{H}_{25} \mathrm{~N}_{2} \mathrm{O}_{3}{ }^{+}\left([\mathrm{M}+\mathrm{H}]^{+}\right)$requires 401.1860; found $401.1859(-0.2$ ppm).

\section{(2R)-(E)-methyl 2-(2-benzoyl-1-phenylhydrazinyl)-4-phenylbut-3-enoate 45}

Following general procedure A, (E)-4-phenylbut-3-enoic acid 16 (64.9 mg, 0.40 mmol), $i \operatorname{Pr}_{2} \mathrm{NEt}(104 \mu \mathrm{L}$, $0.60 \mathrm{mmol})$ and pivaloyl chloride $(74.0 \mu \mathrm{L}, 0.60 \mathrm{mmol})$ in $\mathrm{CH}_{2} \mathrm{Cl}_{2}(2 \mathrm{~mL}), \mathrm{HBTM}-2.1(2 S, 3 R)-23(1.23$ mg, $0.004 \mathrm{mmol}, 1 \mathrm{~mol} \%)$, (NE)-N-(phenylimino)benzamide 60 (84.0 mg, $0.40 \mathrm{mmol})$ and $i \operatorname{Pr}_{2} \mathrm{NEt}(174$ $\mu \mathrm{L}, 1.00 \mathrm{mmol})$ for $15 \mathrm{~min}$ at $\mathrm{rt}$, followed by addition of $\mathrm{MeOH}(2 \mathrm{~mL})$ and stirring for $1 \mathrm{~h}$ at $\mathrm{rt}$ gave, after chromatographic purification (eluent $\mathrm{Et}_{2} \mathrm{O}$ :petrol 40:60) a rotameric mixture (ratio 95:5) of (2R)-45 as an off-white solid (109 mg, 71\%); mp 116-118 ${ }^{\circ} \mathrm{C} ;[\alpha]_{D}^{20}-19.6\left(c 0.25, \mathrm{CH}_{2} \mathrm{Cl}_{2}\right)$; Chiral HPLC Chiralpak IB $\left(5 \%\right.$ IPA:hexane, flow rate $\left.1 \mathrm{~mL} \min ^{-1}, 211 \mathrm{~nm}, 30{ }^{\circ} \mathrm{C}\right) \mathrm{t}_{\mathrm{R}}(2 S): 22.0 \mathrm{~min}, \mathrm{t}_{\mathrm{R}}(2 R): 27.9 \mathrm{~min}, 91 \%$ ee; $v_{\max }$ $(\mathrm{ATR}) / \mathrm{cm}^{-1} 3325(\mathrm{~N}-\mathrm{H}), 3057,2959(\mathrm{C}-\mathrm{H}), 1728(\mathrm{C}=\mathrm{O}), 1693(\mathrm{C}=\mathrm{O}), 1599$; Data for major rotamer: $\delta_{\mathrm{H}}$ $\left(500 \mathrm{MHz}, \mathrm{CDCl}_{3}\right) 3.84\left(3 \mathrm{H}, \mathrm{s}, \mathrm{CH}_{3}\right), 5.50(1 \mathrm{H}, \mathrm{d}, J$ 5.1, C(2)H), $6.54(1 \mathrm{H}, \mathrm{dd}, J$ 16.3, 5.7, CH=CHPh), $6.76(1 \mathrm{H}, \mathrm{d}, J$ 16.3, CH=CHPh), 6.99-7.04 (3H, m, ArH), 7.24-7.41 (7H, m, ArH), $7.44(2 \mathrm{H}, \mathrm{t}, J$ 8.7, $\mathrm{C}(\mathrm{O}) \operatorname{Ar}(3,5) H), 7.54(1 \mathrm{H}, \mathrm{t}, J 7.4, \mathrm{C}(\mathrm{O}) \operatorname{Ar}(4) H), 7.83(2 \mathrm{H}, \mathrm{d}, J 7.4, \mathrm{C}(\mathrm{O}) \operatorname{Ar}(2,6) H), 8.72(1 \mathrm{H}, \mathrm{s}, \mathrm{N} H) ; \delta_{\mathrm{C}}$

$\left(125 \mathrm{MHz}, \mathrm{CDCl}_{3}\right) 52.6\left(\mathrm{CH}_{3}\right), 64.9(C(2)), 114.8(\mathrm{~N} A r C(2,6)), 121.7(\mathrm{~N} A r C(4)), 122.1(C \mathrm{H}=\mathrm{CHPh})$, $126.8(=\mathrm{CH} A r C(4)), 127.2(\mathrm{C}(\mathrm{O}) \operatorname{ArC}(2,6)), 128.3(\operatorname{ArC}), 128.7(\operatorname{ArC}), 128.8(\mathrm{C}(\mathrm{O}) \operatorname{ArC}(3,5)), 129.5$ $\mathrm{N} A r C(3,5)), 132.1(\mathrm{C}(\mathrm{O}) \operatorname{ArC}(4)), 133.0(\mathrm{C}(\mathrm{O}) \operatorname{ArC}(1)), 135.0(\mathrm{CH}=C \mathrm{HPh}), 135.9$ (=CHArC(1)), 148.0 $(\mathrm{N} A r C(1)), 167.6(\mathrm{NHC}=\mathrm{O}), 172.8(\mathrm{MeOC}=\mathrm{O})$; Selected data for minor rotamer: $\delta_{\mathrm{H}}\left(500 \mathrm{MHz}, \mathrm{CDCl}_{3}\right)$ 
$3.73\left(3 \mathrm{H}, \mathrm{s}, \mathrm{CH}_{3}\right), 5.21(1 \mathrm{H}, \mathrm{d}, J 7.2, \mathrm{C}(2) H), 6.02(1 \mathrm{H}, \mathrm{dd}, J 16.1,7.2, \mathrm{CH}=\mathrm{CHPh}), 7.98(1 \mathrm{H}, \mathrm{s}, \mathrm{N} H) ; \delta_{\mathrm{C}}$

$\left(125 \mathrm{MHz}, \mathrm{CDCl}_{3}\right) 52.5\left(\mathrm{CH}_{3}\right), 66.1(C(2)), 115.1(\mathrm{NArC}(2,6)), 137.5(\mathrm{CH}=C \mathrm{HPh}) ; m / z\left(\mathrm{NSI}^{+}\right) 387$ $\left([\mathrm{M}+\mathrm{H}]^{+}, 100 \%\right)$; HRMS $\left(\mathrm{NSI}^{+}\right) \mathrm{C}_{24} \mathrm{H}_{23} \mathrm{~N}_{2} \mathrm{O}_{3}{ }^{+}\left([\mathrm{M}+\mathrm{H}]^{+}\right)$requires 387.1703; found 387.1704 (-0.2 ppm).

\section{(2R)-(Z)-methyl 2-(2-benzoyl-1-phenylhydrazinyl)pent-3-enoate 46}

Following general procedure A, $(Z)$-pent-3-enoic acid $68(40.0 \mathrm{mg}, 0.40 \mathrm{mmol}), i \operatorname{Pr}_{2} \mathrm{NEt}(104 \mu \mathrm{L}, 0.60$ mmol) and pivaloyl chloride $(74.0 \mu \mathrm{L}, 0.60 \mathrm{mmol})$ in $\mathrm{CH}_{2} \mathrm{Cl}_{2}(2 \mathrm{~mL}), \mathrm{HBTM}-2.1(2 S, 3 R)-23(1.23 \mathrm{mg}$, $0.004 \mathrm{mmol}, 1 \mathrm{~mol} \%)$, (NE)-N-(phenylimino)benzamide 60 (84.0 mg, $0.40 \mathrm{mmol})$ and $i \operatorname{Pr}_{2} \mathrm{NEt}(174 \mu \mathrm{L}$, $1.00 \mathrm{mmol})$ for $15 \mathrm{~min}$ at $\mathrm{rt}$, followed by addition of $\mathrm{MeOH}(2 \mathrm{~mL})$ and stirring for $1 \mathrm{~h}$ at $\mathrm{rt}$ gave, after chromatographic purification (eluent $\mathrm{Et}_{2} \mathrm{O}$ :petrol 50:50) a rotameric mixture (ratio 95:5) of (2R)-46 (94:6 $(Z):(E))$ as a colourless oil $(93.7 \mathrm{mg}, 72 \%) ;[\alpha]_{D}^{20}-76.8\left(c 0.5, \mathrm{CH}_{2} \mathrm{Cl}_{2}\right)$; Chiral HPLC Chiralpak AD-H $\left(20 \%\right.$ IPA:hexane, flow rate $\left.1 \mathrm{~mL} \mathrm{~min}^{-1}, 220 \mathrm{~nm}, 30{ }^{\circ} \mathrm{C}\right) \mathrm{t}_{\mathrm{R}}(2 S): 19.1 \mathrm{~min}, \mathrm{t}_{\mathrm{R}}(2 R): 23.6 \mathrm{~min}, 99 \%$ ee; $v_{\max }$ (ATR)/cm ${ }^{-1} 3291(\mathrm{~N}-\mathrm{H}), 2953(\mathrm{C}-\mathrm{H}), 1732$ (C=O Ester), 1674 (C=O Amide), 1599; Data for major isomer (Z) and major rotamer: $\delta_{\mathrm{H}}\left(400 \mathrm{MHz}, \mathrm{CDCl}_{3}\right) 1.88\left(3 \mathrm{H}, \mathrm{dd}, J 7.0,1.8, \mathrm{CH}_{3} \mathrm{CH}=\right), 3.75\left(3 \mathrm{H}, \mathrm{s}, \mathrm{OCH}_{3}\right), 5.48$ $(1 \mathrm{H}, \mathrm{d}, J$ 8.2, $\mathrm{C}(2) H), 5.61-5.67\left(1 \mathrm{H}, \mathrm{m}, \mathrm{CH}=\mathrm{CHCH}_{3}\right), 5.91\left(1 \mathrm{H}, \mathrm{dqd}, J 10.7,7.0,1.0, \mathrm{CH}=\mathrm{CHCH}_{3}\right)$, 6.93-6.99 (3H, m, $\mathrm{NAr}(2,6) H$ and $\mathrm{NAr}(4) H), 7.26-7.30(2 \mathrm{H}, \mathrm{m}, \mathrm{NAr}(3,5) H), 7.46-7.51(2 \mathrm{H}, \mathrm{m}$, $\mathrm{C}(\mathrm{O}) \operatorname{Ar}(3,5) H), 7.54-7.59(1 \mathrm{H}, \mathrm{m}, \mathrm{C}(\mathrm{O}) \operatorname{Ar}(4) H), 7.88-7.91(2 \mathrm{H}, \mathrm{m}, \mathrm{C}(\mathrm{O}) \operatorname{Ar}(2,6) H), 8.67(1 \mathrm{H}, \mathrm{s}, \mathrm{N} H) ; \delta_{\mathrm{C}}$ $\left(100 \mathrm{MHz}, \mathrm{CDCl}_{3}\right) 14.1\left(\mathrm{CH}_{3} \mathrm{CH}=\right), 52.6\left(\mathrm{OCH}_{3}\right), 59.8(C(2)), 114.7(\mathrm{~N} A r C(2,6)), 121.6(\mathrm{~N} A r C(4))$, $121.7\left(\mathrm{CH}=\mathrm{CHCH}_{3}\right), \quad 127.4 \quad(\mathrm{C}(\mathrm{O}) \operatorname{ArC}(2,6)), \quad 128.9 \quad(\mathrm{C}(\mathrm{O}) \operatorname{ArC}(3,5)), \quad 129.5 \quad(\mathrm{~N} A r C(3,5)), \quad 132.2$ $\left(\mathrm{C}(\mathrm{O}) \operatorname{ArC}(4), 132.7 \quad\left(\mathrm{CH}=C \mathrm{HCH}_{3}\right), 132.8(\mathrm{C}(\mathrm{O}) \operatorname{ArC}(1)), 148.3 \quad(\mathrm{~N} A r C(1)), 167.0 \quad(\mathrm{NHC}=\mathrm{O}), 173.6\right.$ $(\mathrm{MeOC}=\mathrm{O}) ; m / z\left(\mathrm{NSI}^{+}\right) 325\left([\mathrm{M}+\mathrm{H}]^{+}, 100 \%\right) ; \mathrm{HRMS}\left(\mathrm{NSI}^{+}\right) \mathrm{C}_{19} \mathrm{H}_{21} \mathrm{~N}_{2} \mathrm{O}_{3}{ }^{+}\left([\mathrm{M}+\mathrm{H}]^{+}\right)$requires 325.1547; found $325.1548(+0.4 \mathrm{ppm})$.

\section{(2R)-(E)-methyl 2-(2-(4-fluorobenzoyl)-1-phenylhydrazinyl)pent-3-enoate 47}

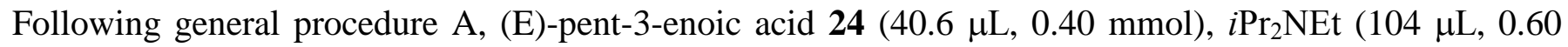
mmol) and pivaloyl chloride $(74.0 \mu \mathrm{L}, 0.60 \mathrm{mmol})$ in $\mathrm{CH}_{2} \mathrm{Cl}_{2}(2 \mathrm{~mL}), \mathrm{HBTM}-2.1(2 S, 3 R)-23(1.23 \mathrm{mg}$, 
$0.004 \mathrm{mmol}, 1 \mathrm{~mol} \%$ ), (NE)-4-fluoro-N-(phenylimino)benzamide 61 (91.2 mg, $0.40 \mathrm{mmol}$ ) and $i \mathrm{Pr}_{2} \mathrm{NEt}$ (174 $\mu \mathrm{L}, 1.00 \mathrm{mmol})$ for $15 \mathrm{~min}$ at $\mathrm{rt}$, followed by addition of $\mathrm{MeOH}(2 \mathrm{~mL})$ and stirring for $1 \mathrm{~h}$ at $\mathrm{rt}$ gave, after chromatographic purification (eluent $\mathrm{Et}_{2} \mathrm{O}$ :petrol 40:60) a rotameric mixture (ratio 92:8) of (2R)-47 as a white solid (109 mg, 79\%); mp 102-104 ${ }^{\circ} \mathrm{C} ;[\alpha]_{D}^{20}-57.8\left(c \quad 0.5, \mathrm{CH}_{2} \mathrm{Cl}_{2}\right)$; Chiral HPLC Chiralpak IB (5\% IPA:hexane, flow rate $\left.1 \mathrm{~mL} \min ^{-1}, 211 \mathrm{~nm}, 30{ }^{\circ} \mathrm{C}\right) \mathrm{t}_{\mathrm{R}}(2 S): 13.2 \mathrm{~min}, \mathrm{t}_{\mathrm{R}}(2 R): 17.1 \mathrm{~min}, 99 \% e e ; v_{\max }$ $(\mathrm{ATR}) / \mathrm{cm}^{-1} 3522(\mathrm{~N}-\mathrm{H}), 2951(\mathrm{C}-\mathrm{H}), 1751(\mathrm{C}=\mathrm{O}), 1661(\mathrm{C}=\mathrm{O}), 1599$; Data for major rotamer: $\delta_{\mathrm{H}}(500$ $\left.\mathrm{MHz}, \mathrm{CDCl}_{3}\right)$ 1.68-1.69 (3H, m, $\left.\mathrm{CH}_{3} \mathrm{CH}=\right), 3.76\left(3 \mathrm{H}, \mathrm{s}, \mathrm{OCH}_{3}\right), 5.21-5.22(1 \mathrm{H}, \mathrm{m}, \mathrm{C}(2) H), 5.74-5.84(2 \mathrm{H}$, m, $\mathrm{CH}=\mathrm{CHCH}_{3}$ and $\left.\mathrm{CH}=\mathrm{CHCH}_{3}\right), 6.91-6.95(3 \mathrm{H}, \mathrm{m}, \mathrm{NAr}(2,6) H$ and $\mathrm{NAr}(4) H), 7.13-7.17(2 \mathrm{H}, \mathrm{t}, J$ 8.4, $\mathrm{NAr}(3,5) H), 7.24-7.27(2 \mathrm{H}, \mathrm{m}, \mathrm{C}(\mathrm{O}) \operatorname{Ar}(3,5) H), 7.84-7.87(2 \mathrm{H}, \mathrm{m}, \mathrm{C}(\mathrm{O}) \operatorname{Ar}(2,6) H), 8.60(1 \mathrm{H}, \mathrm{s}, \mathrm{N} H) ; \delta_{\mathrm{C}}$ $\left(125 \mathrm{MHz}, \mathrm{CDCl}_{3}\right) 18.2\left(\mathrm{CH}_{3} \mathrm{CH}=\right), 52.4\left(\mathrm{OCH}_{3}\right), 64.6(C(2)), 114.8(\mathrm{~N} A r C(2,6)), 116.0(\mathrm{~d}, J) 21.8$, $\mathrm{C}(\mathrm{O}) \operatorname{ArC}(3,5)), 121.7(\mathrm{~N} A r C(4)), 123.7\left(C \mathrm{H}=\mathrm{CHCH}_{3}\right), 129.2(\mathrm{~d}, J 3.5, \mathrm{C}(\mathrm{O}) \operatorname{ArC}(1)), 129.5(\mathrm{~N} A r C(3,5))$, $129.7\left(\mathrm{~d}, J\right.$ 8.7, C(O)ArC(2,6)), $132.4\left(\mathrm{CH}=\mathrm{CHCH}_{3}\right), 148.1(\mathrm{~N} A r C(1)), 165.2(\mathrm{~d}, J$ 252, $\mathrm{C}(\mathrm{O}) \operatorname{ArC}(4))$, 166.4 $(\mathrm{NHC}=\mathrm{O}), 173.4(\mathrm{MeOC}=\mathrm{O}) ; \delta_{\mathrm{F}}\left(376 \mathrm{MHz}, \mathrm{CDCl}_{3}\right)-107.6(\mathrm{Ar} F)$; Selected data for minor rotamer: $\delta_{\mathrm{H}}\left(500 \mathrm{MHz}, \mathrm{CDCl}_{3}\right) 1.61-1.62\left(3 \mathrm{H}, \mathrm{m}, \mathrm{CH}_{3} \mathrm{CH}=\right), 3.65\left(3 \mathrm{H}, \mathrm{s}, \mathrm{OCH}_{3}\right), 4.95(1 \mathrm{H}, \mathrm{d}, J 7.3, \mathrm{C}(2) H), 7.36$ $(2 \mathrm{H}, \mathrm{dd}, J 8.8,7.4, \mathrm{C}(\mathrm{O}) \operatorname{Ar}(3,5) H), 7.58-7.61(2 \mathrm{H}, \mathrm{C}(\mathrm{O}) \operatorname{Ar}(2,6) H) ; \delta_{\mathrm{C}}\left(125 \mathrm{MHz}, \mathrm{CDCl}_{3}\right) 18.2\left(C \mathrm{H}_{3} \mathrm{CH}=\right)$, $52.4\left(\mathrm{OCH}_{3}\right), 66.0(C(2)), 114.8(\mathrm{NArC}(2,6)) ; \delta_{\mathrm{F}}\left(376 \mathrm{MHz}, \mathrm{CDCl}_{3}\right)-108.8(\mathrm{Ar} F) ; m / z\left(\mathrm{NSI}^{+}\right) 343$ $\left([\mathrm{M}+\mathrm{H}]^{+}, 100 \%\right) ; \mathrm{HRMS}\left(\mathrm{NSI}^{+}\right) \mathrm{C}_{19} \mathrm{H}_{20} \mathrm{FN}_{2} \mathrm{O}_{3}{ }^{+}\left([\mathrm{M}+\mathrm{H}]^{+}\right)$requires 343.1452; found $343.1458(+1.6 \mathrm{ppm})$.

\section{(2R)-(E)-methyl 2-(2-(furan-2-yl)-1-phenylhydrazinyl)pent-3-enoate 48}

Following general procedure A, (E)-pent-3-enoic acid $24(40.6 \mu \mathrm{L}, 0.40 \mathrm{mmol}), i \mathrm{Pr}_{2} \mathrm{NEt}(104 \mu \mathrm{L}, 0.60$ mmol) and pivaloyl chloride $(74.0 \mu \mathrm{L}, 0.60 \mathrm{mmol})$ in $\mathrm{CH}_{2} \mathrm{Cl}_{2}(2 \mathrm{~mL}), \mathrm{HBTM}-2.1(2 S, 3 R)-23(1.23 \mathrm{mg}$, $0.004 \mathrm{mmol}, 1 \mathrm{~mol} \%),(\mathrm{NE})-\mathrm{N}-\left(\right.$ phenylimino)furan-2-carboxamide $62(80.0 \mathrm{mg}, 0.40 \mathrm{mmol})$ and $i \mathrm{Pr}_{2} \mathrm{NEt}$ $(174 \mu \mathrm{L}, 1.00 \mathrm{mmol})$ for $15 \mathrm{~min}$ at $\mathrm{rt}$, followed by addition of $\mathrm{MeOH}(2 \mathrm{~mL})$ and stirring for $1 \mathrm{~h}$ at $\mathrm{rt}$ gave, after chromatographic purification (eluent $\mathrm{Et}_{2} \mathrm{O}$ :petrol 50:50) a rotameric mixture (ratio 94:6) of (2R)-48 as a colourless oil $(109 \mathrm{mg}, 87 \%) ;[\alpha]_{D}^{20}-82.4\left(c \quad 0.5, \mathrm{CH}_{2} \mathrm{Cl}_{2}\right)$; Chiral HPLC Chiralpak IB (5\% 
IPA:hexane, flow rate $\left.1 \mathrm{~mL} \mathrm{~min}^{-1}, 211 \mathrm{~nm}, 30{ }^{\circ} \mathrm{C}\right) \mathrm{t}_{\mathrm{R}}(2 S): 16.0 \mathrm{~min}, \mathrm{t}_{\mathrm{R}}(2 R): 22.2 \mathrm{~min}, 99 \%$ ee; $v_{\max }$ $(\mathrm{ATR}) / \mathrm{cm}^{-1} 3335(\mathrm{~N}-\mathrm{H}), 2953(\mathrm{C}-\mathrm{H}), 1730(\mathrm{C}=\mathrm{O}), 1688(\mathrm{C}=\mathrm{O}), 1589$; Data for major rotamer: $\delta_{\mathrm{H}}(500$ $\left.\mathrm{MHz}, \mathrm{CDCl}_{3}\right) 1.70\left(3 \mathrm{H}, \mathrm{dt}, J\right.$ 6.4, 1.3, $\left.\mathrm{CH}_{3} \mathrm{CH}=\right), 3.76\left(3 \mathrm{H}, \mathrm{s}, \mathrm{OCH}_{3}\right), 5.20-5.21(1 \mathrm{H}, \mathrm{m}, \mathrm{C}(2) H), 5.75(1 \mathrm{H}$, ddq, $J$ 15.6, 6.0, 1.5, $\left.\mathrm{CH}=\mathrm{CHCH}_{3}\right), 5.74\left(1 \mathrm{H}, \mathrm{dqd}, J 15.6,6.4,1.2, \mathrm{CH}=\mathrm{CHCH}_{3}\right), 6.56(1 \mathrm{H}, \mathrm{dd}, J 3.5,1.8$, $\mathrm{C}(\mathrm{O}) \operatorname{Ar}(4) H), \quad 6.92-6.95(3 \mathrm{H}, \mathrm{m}, \mathrm{NAr}(2,6) H$ and $\mathrm{NAr}(4) H), \quad 7.24-7.27 \quad(3 \mathrm{H}, \mathrm{m}, \mathrm{NAr}(3,5) H$ and $\mathrm{C}(\mathrm{O}) \operatorname{Ar}(3) H), 7.52(1 \mathrm{H}, \mathrm{dd}, J 1.7,0.8, \mathrm{C}(\mathrm{O}) \operatorname{Ar}(5) H), 8.75(1 \mathrm{H}, \mathrm{s}, \mathrm{NH}) ; \delta_{\mathrm{C}}\left(125 \mathrm{MHz}, \mathrm{CDCl}_{3}\right) 18.2$ $\left(C \mathrm{H}_{3} \mathrm{CH}=\right), 52.4\left(\mathrm{OCH}_{3}\right), 64.7(C(2)), 112.2(\mathrm{C}(\mathrm{O}) \operatorname{ArC}(4)), 114.8(\mathrm{~N} A r C(2,6)), 115.9(\mathrm{C}(\mathrm{O}) \operatorname{ArC}(3))$, $121.6(\mathrm{NArC}(4)), 123.3\left(\mathrm{CH}=\mathrm{CHCH}_{3}\right), 129.4(\mathrm{~N} A r C(3,5)), 132.8\left(\mathrm{CH}=\mathrm{CHCH}_{3}\right), 144.7(\mathrm{C}(\mathrm{O}) \operatorname{ArC}(5))$, $146.6(\mathrm{C}(\mathrm{O}) \operatorname{ArC}(2)), 148.1(\mathrm{~N} A r C(1)), 158.2(\mathrm{NHC}=\mathrm{O}), 173.0(\mathrm{MeOC}=\mathrm{O})$; Selected data for minor rotamer: $\delta_{\mathrm{H}}\left(500 \mathrm{MHz}, \mathrm{CDCl}_{3}\right) 3.69\left(3 \mathrm{H}, \mathrm{s}, \mathrm{OCH}_{3}\right), 5.10(1 \mathrm{H}, \mathrm{d}, J$ 7.1, C(2)H), $6.38(1 \mathrm{H}, \mathrm{dd}, J 3.5,1.7$, $\mathrm{C}(\mathrm{O}) \operatorname{Ar}(3) H) ; \delta_{\mathrm{C}}\left(125 \mathrm{MHz}, \mathrm{CDCl}_{3}\right) 18.1\left(\mathrm{CH}_{3} \mathrm{CH}=\right), 52.4\left(\mathrm{OCH}_{3}\right), 65.5(C(2)), 111.6(\mathrm{C}(\mathrm{O}) \operatorname{ArC}(4))$, $115.0(\mathrm{~N} A r C(2,6)), 129.8(\mathrm{~N} A r C(3,5)), 145.6(\mathrm{C}(\mathrm{O}) \operatorname{ArC}(5)) ; m / z\left(\mathrm{NSI}^{+}\right) 315\left([\mathrm{M}+\mathrm{H}]^{+}, 100 \%\right)$; HRMS $\left(\mathrm{NSI}^{+}\right) \mathrm{C}_{17} \mathrm{H}_{19} \mathrm{~N}_{2} \mathrm{O}_{4}^{+}\left([\mathrm{M}+\mathrm{H}]^{+}\right)$requires 315.1339; found 315.1335 (-1.4 ppm).

$(3 R, 4 R, 5 R)-N^{\prime}-(4-h y d r o x y-5-m e t h y l-2-0 x 00 x o l a n-3-y l)-N^{\prime}-p h e n y l b e n z o h y d r a z i d e \quad$ amine 49 and

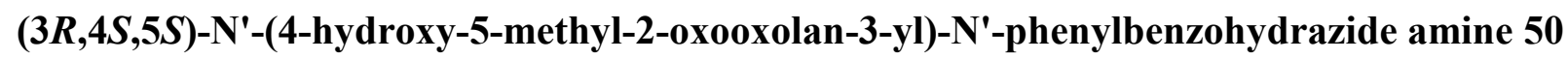

To a solution of hydrazide (2R)-41 (1.12 g, $3.44 \mathrm{mmol})$ in acetone:water (9:1, $40 \mathrm{~mL})$ was added 2,6lutidine $(0.80 \mathrm{~mL}, 6.88 \mathrm{mmol}), \mathrm{N}$-methylmorpholine- $N$-oxide $(0.60 \mathrm{~g}, 5.16 \mathrm{mmol})$ and $\mathrm{OsO}_{4}(4 \% \mathrm{wt}$ in $\mathrm{H}_{2} \mathrm{O}, 0.44 \mathrm{~mL}, 0.07 \mathrm{mmol}$ ) and the reaction mixture was stirred at $\mathrm{rt}$ for $5 \mathrm{~h}$ after which time if was quenched by addition of sat. aq. $\mathrm{Na}_{2} \mathrm{~S}_{2} \mathrm{O}_{3}$. The reaction mixture was extracted with EtOAc (x 3) and the combined organic fractions were washed with $\mathrm{HCl}\left(2 \mathrm{M}\right.$ in $\left.\mathrm{H}_{2} \mathrm{O}\right)$, dried $\left(\mathrm{MgSO}_{4}\right)$, filtered and concentrated in vacuo to give a mixture of crude diols $(2 R, 3 R, 4 R)-\mathbf{4 9}$ and $(2 R, 3 S, 4 S)$-50 which were used directly in the next reaction without purification. The crude reaction mixture was dissolved in $\mathrm{CH}_{2} \mathrm{Cl}_{2}(50 \mathrm{~mL})$ and treated with $p$-toluenesulfonic acid $(0.65 \mathrm{~g}, 3.44 \mathrm{mmol})$. The reaction mixture was stirred at $\mathrm{rt}$ for $2 \mathrm{~h}$ before being quenched by addition of $\mathrm{H}_{2} \mathrm{O}$. The reaction mixture was extracted with $\mathrm{CH}_{2} \mathrm{Cl}_{2}(\mathrm{x} 3)$ and the 
combined organic fractions were dried $\left(\mathrm{MgSO}_{4}\right)$, filtered and concentrated in vacuo to give crude lactones $(3 R, 4 R, 5 R)-\mathbf{4 9}$ and $(3 R, 4 S, 5 S)-\mathbf{5 0}(70: 30 \mathrm{dr})$.

Major diastereoisomer: Chromatographic purification (eluent $\mathrm{Et}_{2} \mathrm{O}$ :petrol 60:40 to 100\% $\mathrm{Et}_{2} \mathrm{O}$ ) gave lactone $(3 R, 4 R, 5 R)-49(>99: 1 \mathrm{dr})$ as a white solid $(0.59 \mathrm{~g}, 53 \%) ; \mathrm{mp} 58-60{ }^{\circ} \mathrm{C} ;[\alpha]_{D}^{20}+37.0(c 0.5$, $\left.\mathrm{CH}_{2} \mathrm{Cl}_{2}\right)$; Chiral HPLC Chiralcel OJ-H (10\% IPA:hexane, flow rate $\left.1 \mathrm{~mL} \mathrm{~min}{ }^{-1}, 211 \mathrm{~nm}, 30{ }^{\circ} \mathrm{C}\right)$ $\mathrm{t}_{\mathrm{R}}(3 S, 4 S, 5 S): 13.5 \mathrm{~min}, \mathrm{t}_{\mathrm{R}}(3 R, 4 R, 5 R): 19.3 \mathrm{~min}, 99 \%$ ee; $v_{\max }(\mathrm{ATR}) / \mathrm{cm}^{-1} 3306(\mathrm{~N}-\mathrm{H}$ and O-H), $2980(\mathrm{C}-$ H), 1767 (lactone $\mathrm{C}=\mathrm{O}), 1661$ (amide $\mathrm{C}=\mathrm{O}), 1597 ; \delta_{\mathrm{H}}\left(500 \mathrm{MHz}, \mathrm{CDCl}_{3}\right) 1.44\left(3 \mathrm{H}, \mathrm{d}, J\right.$ 6.3, $\left.\mathrm{CH}_{3}\right), 4.73$ $4.79(3 \mathrm{H}, \mathrm{m}, \mathrm{C}(3) H, \mathrm{C}(4) H$ and $\mathrm{C}(5) H), 5.02(1 \mathrm{H}$, br s, OH), 6.93-6.96 (3H, m, NAr(2,6)H and $\mathrm{NAr}(4) H)$, $7.24(2 \mathrm{H}, \mathrm{td}, J$ 9.1, 1.7, $\mathrm{NAr}(3,5) H), 7.43-7.46(2 \mathrm{H}, \mathrm{m}, \mathrm{C}(\mathrm{O}) \operatorname{Ar}(3,5) H), 7.58(1 \mathrm{H}, \mathrm{tt}, J$ 7.5, 1.4, $\mathrm{C}(\mathrm{O}) \operatorname{Ar}(4) H), 7.79-7.81(2 \mathrm{H}, \mathrm{m}, \mathrm{C}(\mathrm{O}) \operatorname{Ar}(2,6) H), 8.58(1 \mathrm{H}$, br s, $\mathrm{N} H) ; \delta_{\mathrm{C}}\left(125 \mathrm{MHz}, \mathrm{CDCl}_{3}\right) 14.9\left(C \mathrm{H}_{3}\right)$, $66.8(C(3)), 69.3(C(4)), 77.3(C(5)), 114.7(\mathrm{~N} A r C(2,6)), 121.7(\mathrm{~N} A r C(4)), 127.6(\operatorname{ArC}), 129.0(\operatorname{ArC})$, $129.5(\operatorname{ArC}), 131.4 \quad(\mathrm{C}=\mathrm{O} A r C(1)), 133.0 \quad(\mathrm{C}=\mathrm{O} A r C(4)), 148.0 \quad(\mathrm{~N} A r C(1)), 168.8 \quad(\mathrm{NHC}=\mathrm{O}), 172.0$ $(\mathrm{MeOC}=\mathrm{O}) ; m / z\left(\mathrm{NSI}^{+}\right) 327\left([\mathrm{M}+\mathrm{H}]^{+}, 86 \%\right) ; \mathrm{HRMS}\left(\mathrm{NSI}^{+}\right) \mathrm{C}_{18} \mathrm{H}_{19} \mathrm{~N}_{2} \mathrm{O}_{4}{ }^{+}\left([\mathrm{M}+\mathrm{H}]^{+}\right)$requires 327.1339; found $327.1345(+1.7 \mathrm{ppm})$.

Minor diastereoisomer: Chromatographic purification (eluent $\mathrm{Et}_{2} \mathrm{O}$ :petrol 60:40 to 100\% $\mathrm{Et}_{2} \mathrm{O}$ ) gave lactone $(3 R, 4 S, 5 S)-50(>99: 1 \mathrm{dr})$ as a white solid $(0.28 \mathrm{~g}, 25 \%) ; \mathrm{mp} 110-112{ }^{\circ} \mathrm{C} ;[\alpha]_{D}^{20}-266.4(c 0.5$, $\mathrm{CH}_{2} \mathrm{Cl}_{2}$ ); Chiral HPLC Chiralpak IA (40\% IPA:hexane, flow rate $\left.1 \mathrm{~mL} \mathrm{~min}{ }^{-1}, 211 \mathrm{~nm}, 30{ }^{\circ} \mathrm{C}\right)$ $\mathrm{t}_{\mathrm{R}}(3 S, 4 R, 5 R): 8.9 \mathrm{~min}, \mathrm{t}_{\mathrm{R}}(3 R, 4 S, 5 S): 14.8 \mathrm{~min}, 99 \%$ ee; $v_{\max }(\mathrm{ATR}) / \mathrm{cm}^{-1} 3238(\mathrm{~N}-\mathrm{H}$ and O-H), $2938(\mathrm{C}-$ H), 1776 (lactone $\mathrm{C}=\mathrm{O}$ ), 1668 (amide $\mathrm{C}=\mathrm{O}), 1597,1510 ; \delta_{\mathrm{H}}\left(500 \mathrm{MHz}, \mathrm{CDCl}_{3}\right) 1.51\left(3 \mathrm{H}, \mathrm{d}, J 6.3, \mathrm{CH}_{3}\right)$, 4.61-4.65 (2H, m, C(4)H and $\mathrm{C}(5) H), 4.76(1 \mathrm{H}, \mathrm{d}, J 4.3, \mathrm{C}(3) H), 4.95(1 \mathrm{H}, \mathrm{s}, \mathrm{OH}), 6.88(2 \mathrm{H}, \mathrm{d}, J 8.0$, $\mathrm{NAr}(2,6) H), 6.97(1 \mathrm{H}, \mathrm{t}, J$ 7.4, NAr(4)H), $7.30(2 \mathrm{H}, \mathrm{tt}, J$ 7.2, 1.8, NAr(3,5)H), 7.48-7.51 $(2 \mathrm{H}, \mathrm{m}$, $\mathrm{C}(\mathrm{O}) \operatorname{Ar}(3,5) H), 7.58-7.62(1 \mathrm{H}, \mathrm{m}, \mathrm{C}(\mathrm{O}) \operatorname{Ar}(4) H), 7.89-7.91(2 \mathrm{H}, \mathrm{m}, \mathrm{C}(\mathrm{O}) \operatorname{Ar}(2,6) H), 8.92(1 \mathrm{H}, \mathrm{s}, \mathrm{N} H) ; \delta_{\mathrm{C}}$ $\left(125 \mathrm{MHz}, \mathrm{CDCl}_{3}\right) 13.9\left(\mathrm{CH}_{3}\right), 67.7(C(3)), 70.3(C(4)), 79.4(C(5)), 113.0(\mathrm{~N} A r C(2,6)), 121.5(\mathrm{~N} A r C(4))$, $127.6(\operatorname{ArC}), 129.1(\operatorname{ArC}), 129.8(\operatorname{ArC}), 131.4(\mathrm{C}=\mathrm{O} A r C(1)), 133.1(\mathrm{C}=\mathrm{O} A r C(4)), 147.3(\mathrm{~N} A r C(1)), 168.9$ 
$(\mathrm{NHC}=\mathrm{O}), 172.0(\mathrm{MeOC}=\mathrm{O}) ; \mathrm{m} / z\left(\mathrm{NSI}^{+}\right) 327\left([\mathrm{M}+\mathrm{H}]^{+}, 100 \%\right) ; \mathrm{HRMS}\left(\mathrm{NSI}^{+}\right) \mathrm{C}_{18} \mathrm{H}_{19} \mathrm{~N}_{2} \mathrm{O}_{4}{ }^{+}\left([\mathrm{M}+\mathrm{H}]^{+}\right)$ requires 327.1339; found $327.1346(+2.0 \mathrm{ppm})$.

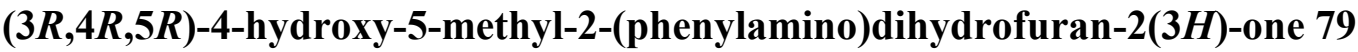

To a solution of lactone $(3 R, 4 R, 5 R)-49(65.2 \mathrm{~g}, 0.20 \mathrm{mmol})$ in $\mathrm{MeOH}(2 \mathrm{~mL})$ at $-78{ }^{\circ} \mathrm{C}$ was added $\mathrm{SmI}_{2}$ $(0.1 \mathrm{M}$ in THF, $6.00 \mathrm{~mL}, 0.60 \mathrm{mmol})$ and the reaction mixture was stirred at $-78{ }^{\circ} \mathrm{C}$ for 10 minutes after which time if was quenched by addition of sat. aq. $\mathrm{NaHCO}_{3}$. The reaction mixture was extracted with EtOAc (x 3) and the combined organic fractions were dried $\left(\mathrm{MgSO}_{4}\right)$, filtered and concentrated in vacuo. Chromatographic purification (eluent $\mathrm{Et}_{2} \mathrm{O}$ :petrol 60:40) gave lactone $(3 R, 4 R, 5 R)-79$ as a colourless oil (29.0 mg, 70\%); $[\alpha]_{D}^{20}+18.0\left(c\right.$ 0.1, $\left.\mathrm{CH}_{2} \mathrm{Cl}_{2}\right) ; v_{\max }(\mathrm{ATR}) / \mathrm{cm}^{-1} 3381(\mathrm{~N}-\mathrm{H}$ or O-H), $2986(\mathrm{C}-\mathrm{H}), 1761$ $(\mathrm{C}=\mathrm{O}), 1603,1499 ; \delta_{\mathrm{H}}\left(500 \mathrm{MHz}, \mathrm{CDCl}_{3}\right) 1.45\left(3 \mathrm{H}, \mathrm{d}, J 6.8, \mathrm{CH}_{3}\right), 2.74(1 \mathrm{H}, \mathrm{d}, J 4.9, \mathrm{OH}$ or $\mathrm{NH}), 4.13$ $(1 \mathrm{H}, \mathrm{s}, \mathrm{OH}$ or $\mathrm{NH}), 4.18-4.19(1 \mathrm{H}, \mathrm{m}, \mathrm{C}(3) H), 4.41(1 \mathrm{H}, \mathrm{td}, J 7.0,4.8, \mathrm{C}(4) H), 4.73$ (1H, app. quintet, $J$ 6.7, C(5)H), 6.80-6.85 (3H, m, NAr(2,6)H and $\operatorname{NAr}(4) H), 7.19-7.23(2 \mathrm{H}, \mathrm{m}, \mathrm{NAr}(3,5) H) ; \delta_{\mathrm{C}}(125 \mathrm{MHz}$, $\left.\mathrm{CDCl}_{3}\right) 14.7\left(\mathrm{CH}_{3}\right), 59.6(C(3)), 74.9(C(4)), 77.4(C(5)), 114.1(\mathrm{~N} A r C(2,6)), 119.6(\mathrm{~N} A r C(4)), 129.6$ $(\mathrm{N} A r C(3,5)), 146.8(\mathrm{~N} A r C(1)), 174.2 \quad(C=\mathrm{O}) ; m / z \quad\left(\mathrm{NSI}^{+}\right) 208 \quad\left([\mathrm{M}+\mathrm{H}]^{+}, \quad 100 \%\right) ; \quad \mathrm{HRMS} \quad\left(\mathrm{NSI}^{+}\right)$ $\mathrm{C}_{11} \mathrm{H}_{14} \mathrm{NO}_{3}{ }^{+}\left([\mathrm{M}+\mathrm{H}]^{+}\right)$requires 208.0968; found $208.0968(-0.1 \mathrm{ppm})$.

\section{$(3 R, 4 S, 5 S)-4-h y d r o x y-5-m e t h y l-2-(p h e n y l a m i n o) d i h y d r o f u r a n-2(3 H)$-one 80}

To a solution of lactone $(3 R, 4 S, 5 S)-\mathbf{5 0}(65.2 \mathrm{~g}, 0.20 \mathrm{mmol})$ in $\mathrm{MeOH}(2 \mathrm{~mL})$ at $-78{ }^{\circ} \mathrm{C}$ was added $\mathrm{SmI}_{2}$ $(0.1 \mathrm{M}$ in $\mathrm{THF}, 6.00 \mathrm{~mL}, 0.60 \mathrm{mmol})$ and the reaction mixture was stirred at $-78{ }^{\circ} \mathrm{C}$ for 10 minutes after which time if was quenched by addition of sat. aq. $\mathrm{NaHCO}_{3}$. The reaction mixture was extracted with EtOAc (x 3) and the combined organic fractions were dried $\left(\mathrm{MgSO}_{4}\right)$, filtered and concentrated in vacuo. Chromatographic purification (eluent $\mathrm{Et}_{2} \mathrm{O}$ :petrol 60:40) gave lactone $(3 R, 4 S, 5 S)-\mathbf{8 0}$ as a colourless oil

(31.4 mg, 76\%); $[\alpha]_{D}^{20}-98.0\left(c \quad 0.1, \mathrm{CH}_{2} \mathrm{Cl}_{2}\right) ; v_{\max }(\mathrm{ATR}) / \mathrm{cm}^{-1} 3389(\mathrm{~N}-\mathrm{H}$ or O-H), $2982(\mathrm{C}-\mathrm{H}), 1761$ $(\mathrm{C}=\mathrm{O}), 1603,1506 ; \delta_{\mathrm{H}}\left(400 \mathrm{MHz}, \mathrm{CDCl}_{3}\right) 1.47\left(3 \mathrm{H}, \mathrm{d}, J 6.5, \mathrm{CH}_{3}\right), 2.76(1 \mathrm{H}$, br s, OH or $\mathrm{NH}), 4.08(1 \mathrm{H}$, d, J 4.4, C(3)H), $4.40(1 \mathrm{H}, \mathrm{dd}, J 4.4,2.9, \mathrm{C}(4) H), 4.58(1 \mathrm{H}, \mathrm{qd}, J 6.5,2.9, \mathrm{C}(5) H), 6.67-6.70(2 \mathrm{H}, \mathrm{m}$, 
NAr(2,6)H), $6.84\left(1 \mathrm{H}, \mathrm{tt}, J\right.$ 7.4, 1.0, NAr(4)H), 7.16-7.21 (2H, m, NAr(3,5)H); $\delta_{\mathrm{C}}\left(100 \mathrm{MHz}, \mathrm{CDCl}_{3}\right) 13.8$

$\left(C \mathrm{H}_{3}\right), 60.4(C(3)), 69.8(C(4)), 78.6(C(5)), 114.2(\mathrm{NArC}(2,6)), 120.5(\mathrm{~N} A r C(4)), 129.6(\mathrm{~N} A r C(3,5))$,

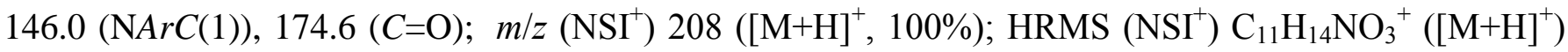
requires 208.0968; found $208.0968(-0.1 \mathrm{ppm})$.

Acknowledgements: We thank the Royal Society for a University Research Fellowships (A.D.S) and The Carnegie Trust for the Universities of Scotland (L.C.M) for funding. The research leading to these results has received funding from the European Research Council under the European Union's Seventh Framework Programme (FP7/2007-2013) / ERC grant agreement $n^{\circ} 279850$. We also thank the EPSRC National Mass Spectrometry Service Centre (Swansea). SMS Funding?

Supporting Information Available: $\beta$-lactam epimerization studies, Assignments of aza sugar relative configurations, X-ray structural data, spectral and HPLC data for all new compounds are provided. This material is available free of charge via the Internet at http://pubs.acs.org.

\section{REFERENCES}

(1) For selected reviews on dienamine catalysis, see: (a) Jensen, K. L.; Dickmeiss, G.; Jiang, H.; Albrecht,Ł.; Jørgensen, K. A. Acc. Chem. Res., 2012, 45, 248-264; (b) Nielsen, M.; Worgull, D.; Zweifel, T.; Gschwend, B.; Bertelsen, S.; Jørgensen, K. A. Chem. Commun., 2011, 47, 632-649; (c) Ramachary, D. B.; Reddy, Y. V. Eur. J. Org. Chem. 2012, 865-887.

(2) (a) Tiseni, P. S.; Peters, R. Angew. Chem. Int. Ed. 2007, 46, 5325-5328; (b) Tiseni, P. S.; Peters, R. Org. Lett. 2008, 10, 2019-2022; (c) Tiseni, P. S.; Peters, R. Chem. Eur. J. 2010, 16, 2503-2517.

(3) (a) Shen, L-T.; Sun, L-H.; Ye, S. J. Am. Chem. Soc. 2011, 133, 15894-15897; (b) Shen, L-T.; Jia, WQ.; Ye, S. Angew. Chem. Int. Ed. 2013, 52, 585-588.

(4) Shen, L-T.; Shao, P-L.; Ye, S. Adv. Synth. Catal. 2011, 353, 1943-1948. 
(5) Mo, J.; Chen, X.; Chi, Y. R. J. Am. Chem. Soc. 2012, 134, 8810-8813.

(6) Chen, X.; Yang, S.; Song, B-A.; Chi, Y. R. Angew. Chem. Int. Ed. 2013, 52, 11134-11137.

(7) Xu, J.; Jin, Z.; Chi, Y. R. Org. Lett. 2013, 15, 5028-5031.

(8) Yao, C.; Xiao, Z.; Liu, R.; Li, T.; Jiao, W.; Yu, C. Chem. Eur. J., 2013, 19, 456-459.

(9) C1-azolium dienolates accessed from $\alpha, \beta$-unsaturated acid chlorides 4 may also be formed via a mechanism involving dehydrohalogenation to form a vinyl ketene intermediate which is subsequently intercepted by the NHC.

(10) Zhao, Y-M.; Cheung, M. S.; Lin, Z.; Sun, J. Angew. Chem. Int. Ed. 2012, 51, 10359-10363.

(11) Chen, X-Y.; Xia, F.; Chen, J-T.; Ye, S. Angew. Chem. Int. Ed. 2013, 52, 10644-10647.

(12) For the pioneering work of Romo regarding the activation of carboxylic acids towards intramolecular nucleophile catalysed aldol-lactonisation (NCAL) reactions see: (a) Cortez, G. S.; Tennyson, R. L.; Romo, D. J. Am. Chem. Soc. 2001, 123, 7945-7946; (b) Cortez, G. S.; Oh, S. H.; Romo, D. Synthesis 2001, 1731-1736; (c) Oh, S. H.; Cortez, G. S.; Romo, D. J. Org. Chem. 2005, 70, 2835-2838; (d) Henry-Riyad, H.; Lee. C.; Purohit, V. C.; Romo, D. Org. Lett. 2006, 8, 4363-4366; (e) Ma, G.; Nguyen, H.; Romo, D. Org. Lett. 2007, 9, 2143-2146; (f) Purohit, V. C.; Matla, A. S.; Romo, D. J. Am. Chem. Soc. 2008, 130, 10478-10479; (g) Nguyen, H.; Ma, G.; Romo, D. Chem. Commun. 2010, 46, 48034805; (h) Morris, K. A.; Arendt, K. M.; Oh, S. H.; Romo, D. Org. Lett. 2010, 12, 3764-3767; (i) Leverett, C.; Purohit, V. C.; Romo, D. Angew. Chem. Int. Ed. 2010, 49, 9479-9483; (j) Nguyen, H.; Oh, S.; HenryRiyad, H.; Sepulveda, D.; Romo, D. Org. Synth. 2011, 88, 121;-137 (k) Nguyen, H.; Ma, G.; Gladysheva, T.; Fremgen, T.; Romo, D. J. Org. Chem. 2011, 76, 2-12; (1) Liu, G.; Romo, D. Angew. Chem. Int. Ed. 2011, 50, 7537-7540; (m) Leverett, C. A.; Purohit, V. C.; Johnson, A. G.; Davis, R. L.; Tantillo, D. J.; Romo, D. J. Am. Chem. Soc. 2012, 134, 13348-13356; (n) Liu, G.; Shirley, M. E.; Romo, D. J. Org. Chem. 2012, 77, 2496-2500. 
(13) For a recent review detailing the use of isothioureas in nucleophilic catalysis see Taylor, J. E.; Bull, S. D.; Williams, J. M. J. Chem. Soc. Rev. 2012, 14, 2109-2121.

(14) For pioneering work demonstrating the utility of isothioureas as efficient $O$-acyl transfer agents see: (a) Birman, V. B.; Li, X. Org. Lett., 2006, 8, 1351-1354; (b) Kobayashi, M.; Okamoto, S. Tetrahedron Lett., 2006, 47, 4347-4350.

(15) For an excellent review of ammonium enolate chemistry see Gaunt, M. J.; Johansson, C. C. C. Chem. Rev., 2007, 107, 5596-5605.

(16) For examples see: (a) Belmessieri, D.; Morrill, L. C.; Simal, C.; Slawin, A. M. Z.; Smith, A. D. J. Am. Chem. Soc. 2011, 133, 2714-2720; (b) Simal, C.; Lebl, T.; Slawin, A. M. Z.; Smith, A. D. Angew. Chem. Int. Ed. 2012, 51, 3653-3657; (c) Morrill, L. C.; Lebl, T.; Slawin, A. M. Z.; Smith, A. D. Chem. Sci. 2012, 3, 2088-2093; (d) Morrill, L. C.; Douglas, J.; Lebl, T.; Slawin, A. M. Z.; Fox D. J.; Smith, A. D. Chem. Sci., 2013, 4, 4146-4155; (e) Belmessieri, D.; Cordes, D. B.; Slawin, A. M. Z.; Smith, A. D. Org. Lett., 2013, 15, 3472-3475; (f) D. G. Stark, L. C. Morrill, P-P. Yeh, T. J. O’Riordan, Angew. Chem. Int. $E d .$, 2013, 52, 11642-11646.

(17) In Ref 16(c), using specific reaction conditions, selected non-arylacetic acids could be used - namely (thiophenyl)acetic acid, methoxyacetic acid and 3-phenylpropionic acid - when a highly reactive $N$-aryl- $N$ aroyldiazene Michael acceptor was used.

(18) For a recent report from our research group detailing the use of 2-arylacetic anhydrides as ammonium enolate precusors see Morrill, L. C.; Ledingham, L. A.; Couturier, J-P.; Bickel, J.; Harper, A. D.; Fallan, C.; Smith, A. D. Org. Biomol. Chem. 2013, 12, 624-636.

(19) Smith, S. R.; Douglas, J.; Prevet, H.; Shapland, P.; Slawin, A. M. Z.; Smith, A. D. J. Org. Chem. preceeding publication.

(20) All experiments were carried out under air using bench grade solvents as standard. 
(21) Other carboxylic acid starting materials, namely (E)-3-phenylbut-2-enoic acid 70 and $(E)-3,4-$ diphenylbut-2-enoic acid $\mathbf{7 2}$ were tested to attempt to access ammonium dienolates which react at the $\gamma$ position but none were successful.

(22) The relative configurations of $\beta$-lactones (anti 21, syn 73) and $\beta$-lactams (anti 22, syn 74) in table 1 were confirmed unambiguously by X-ray crystallography. Crystallographic data for all diastereoisomers 21, 22, 73 and 74 have been deposited with the Cambridge Crystalographic Data Centre as supplementary publication numbers CCDC 968689, 968690, 968692 and 968693.

(23) Although we describe these reactions as formal [2+2] cycloadditions, they may also be considered as intermolecular enolate-imine cyclisation reactions, or Gilman-Speeter reactions. For representative examples see: (a) Hiroki, F.; Kanai, M.; Kambara, T.; Iida, A.; Tomioka, K. J. Am. Chem. Soc. 1997, 119, 2060-2061; (b) Tomioka, K.; Fujieda, H.; Hayashi, S.; Hussein, M. A.; Kambara, T.; Nomura, Y.; Kanai, M.; Koga, K. Chem. Commun. 1999, 715-716; (c) Hussein, M. A.; Iida, A.; TOmioka, K. Tetrahedron 1999, 55, 11219-11228; (d) Kambara, T.; Tomioka, K. Chem. Pharm. Bull. 200048 , 1577-1580; (e) Evans C. D.; Mahon, M. R.; Andrews, P. C.; Muir, J.; Bull, S. D. Org. Lett. 2011,13, 6276-6279. For the Gilman-Speeter reaction see: Gilman, H.; Speeter, M. J. Am. Chem. Soc., 1943, 65, $2255-2256$.

(24) For selected alternative methods for accessing $\beta$-lactams bearing an olefin at C(3), see: (a) Torii, S.; Okumoto, H.; Sadakane, M.; Hai, A. K. M. A.; Tanaka, H. Tetrahedron Lett. 1993, 34, 6553-6556; (b) Taggi, A. E.; Hafez, A. M.; Wack, H.; Young, B.; Ferraris, D.; Lectka, T. J. Am. Chem. Soc. 2002, 124, 6626-6635; (c) Fontana, F.; Tron, G. C.; Barbero, N.; Ferrini, S.; Thomas, S. P.; Aggarwal, V. K. Chem. Commun. 2010, 46, 267-269; (d) Xia, P.; Qian, B.; Huang, H. Tetrahedron Lett. 2012, 53, 1613-1616. For discussions regarding the biological importance of the $\beta$-lactam motif, see: (a) Morin, R. B.; Gorman, M. Chemistry and biology of beta-lactam antibiotics; Academic Press, 1982; (b) Jastrzebski, J. T. B. H.; VanKoten, G. In Comprehensive Heterocyclic Chemistry II; Editors-in-Chief: Alan, R. K., Charles, W. 
R., Eric F.V. Scriven - Editors-in-Chief: Alan R. Katritzky, C. W. R., Eric, F. V. S., Eds.; Pergamon: Oxford, 1996, p 623-658; (c) Zhang, T. Y.; Hatfield, L. D. In Comprehensive Heterocyclic Chemistry II; Editors-in-Chief: Alan, R. K., Charles, W. R., Eric F.V. Scriven - Editors-in-Chief: Alan R. Katritzky, C. W. R., Eric, F. V. S., Eds.; Pergamon: Oxford, 1996, p 591-622.

(25) Racemic samples of all $\beta$-lactams in tables 2 and 3 were prepared using achiral catalyst DHPB 17.

(26) The absolute configuration of syn- $\beta$-lactam 27 was confirmed unambiguously by X-ray crystallography as $(3 S, 4 S)$. Crystallographic data for $\mathbf{2 7}$ has been deposited with the Cambridge Crystalographic Data Centre as supplementary publication number CCDC 968691.

(27) See SI for full details of the epimerization experiment.

(28) Poor conversion to $\beta$-lactams 28 and 29 is observed when the reactions are carried out at $-78{ }^{\circ} \mathrm{C}$.

(29) All reactions quoted at $-78{ }^{\circ} \mathrm{C}$ were also carried out at rt, typically giving lower enantioselectivities for $\beta$-lactam products. See SI for these results.

(30) Racemic ( \pm -36 was prepared using ( \pm )-HBTM-2.1 23. Racemic samples of all products in Table 4 were made using ( \pm )-HBTM-2.1 23 as a catalyst.

(31) The minor diastereoisomer was formed in $15 \%$ ee. The enantioselectivities of all minor diastereoisomers in Table 4 can be found in the SI.

(32) The relative and absolute configuration of $\delta$-lactone $\mathbf{3 6}$ was assigned by comparison with the literature Ref 16(d). All other products in Table 4 were assigned by analogy.

(33) The initial heterocyclic products were immediately ring-opened with methanol due to their instability towards chromatographic purification on silica.

(34) The absolute configuration of hydrazide $\mathbf{4 1}$ was assigned by comparison with the literature Ref 16(c). All other products in Table 5 were assigned by analogy. 
(35) Racemic ( \pm )-41 was prepared using the achiral isothiourea DHPB 17. Racemic samples of all products in Table 5 were made using DHPB 17 as a catalyst.

(36) The N-N bond in lactones 49 and $\mathbf{5 0}$ were cleaved using $\mathrm{SmI}_{2}$ in $70 \%$ and $76 \%$ yield respectively. Coupling constant analysis in addition to NOE studies of these products allowed the relative configurations of lactones $\mathbf{4 9}$ and $\mathbf{5 0}$ to be assigned. See SI for details.

(37) McKay, W. R.; Proctor, G. R.; J. Chem. Soc. Perkin Trans 1, 1981, 2435-2442.

(38) Smith III, A. B.; Toder, B. H.; Branca, S. J.; Dieter, R. K.; J. Am. Chem. Soc., 1981, 103, 19962008.

(39) Augustyns, K.; Haemers, A.; Kertesz, I.; Senten, K.; Van Der Veken, P.; De Meester, I.; Lambeir, A-M.; Maes, M-B.; Scharpe, S.; J. Med. Chem., 2005, 48, 1768-1780.

(40) Kantorowski, E. J.; Le, D. D.; Hunt, C. J.; Barry-Holson, K. Q.; Lee, J. P.; Ross, L. N. J. Org. Chem., 2008, 73, 1593-1596.

(41) Miyashi, T.; Nishizawa, Y.; Fujii, Y.; Yamakawa, K.; Kamata, M.; Akao, S.; Mukai, T. J. Am. Chem. Soc., 1986, 108, 1617-1632.

(42) Smith, S. M.; Thacker, N. C.; Takacs, J. M. J. Am. Chem. Soc., 2008, 130, 3734-3735.

(43) Loy, N. S. Y.; Singh, A.; Xu, X.; Park, C-M. Angew. Chem. Int. Ed., 2013, 52, 2212-2216.

(44) Smejkal, T.; Breit, B. Angew. Chem. Int. Ed., 2008, 47, 311-315.

(45) Maleczka, R. E.; Rahaim, R. J. Org. Lett., 2011, 13, 584-587.

(46) Tellier, F.; Sauvetre, R. Tetrahedron Letters, 1993, 34, 5433-5436.

(47) Takimoto, M.; Shimizu, K.; Mori, M. Org. Lett., 2001, 3, 3345-3347.

(48) Duan, X-G.; Duan, X-L.; Rees, C. W. J. Chem Soc., Perkin Trans. 1, 1997, 2831-2836. 
(49) Abarbri, M.; Thibonnet, J; Parrain, J-L.; Duchene, A.; Synthesis, 2002, 543-551. 